\title{
WestVirginiaUniversity
}

THE RESEARCH REPOSITORY @ WVU

Graduate Theses, Dissertations, and Problem Reports

2003

\section{Potential field theory and its applications to classical mechanical problems}

Shannon Rae Stillwagon

West Virginia University

Follow this and additional works at: https://researchrepository.wvu.edu/etd

\section{Recommended Citation}

Stillwagon, Shannon Rae, "Potential field theory and its applications to classical mechanical problems" (2003). Graduate Theses, Dissertations, and Problem Reports. 1400.

https://researchrepository.wvu.edu/etd/1400

This Thesis is protected by copyright and/or related rights. It has been brought to you by the The Research Repository @ WVU with permission from the rights-holder(s). You are free to use this Thesis in any way that is permitted by the copyright and related rights legislation that applies to your use. For other uses you must obtain permission from the rights-holder(s) directly, unless additional rights are indicated by a Creative Commons license in the record and/ or on the work itself. This Thesis has been accepted for inclusion in WVU Graduate Theses, Dissertations, and Problem Reports collection by an authorized administrator of The Research Repository @ WVU. For more information, please contact researchrepository@mail.wvu.edu. 


\title{
POTENTIAL FIELD THEORY AND ITS APPLICATIONS TO CLASSICAL MECHANICAL PROBLEMS
}

\author{
by \\ Shannon Rae Stillwagon \\ A thesis submitted to the \\ College of Engineering and Mineral Resources \\ at West Virginia University \\ in partial fulfillment of the requirements for the \\ degree of

\section{Master of Science} \\ Department: Mechanical and Aerospace Engineering \\ Major: Mechanical Engineering
}

\author{
West Virginia University \\ Morgantown, West Virginia \\ 2003
}

Keywords: Potential Field Theory, Scale, Strength, Laplace's equation, Nanotechnology 


\title{
Abstract \\ POTENTIAL FIELD THEORY AND ITS APPLICATIONS TO CLASSICAL MECHANICAL PROBLEMS
}

\author{
Shannon Rae Stillwagon
}

\begin{abstract}
Advances in many scientific fields are expected to come from work in nanotechnology. Engineering at nano-scales presents novel problems that classical mechanics cannot solve. Many engineers are uncomfortable designing at this level because classical or continuum mechanics does not apply and quantum mechanics is said to apply in a tangible way. There are unique opportunities to contribute to the design, controls, and analysis of systems that are particularly suited to mechanical engineering. Within the derivations of classical mechanics are assumptions that limit its use to bulk engineering. These assumptions are examined to determine what principles can be extended to smaller scales. To allow engineers to do their job at these scales, it is necessary to understand strength and how changing scales affects the strength of material this leads directly to sets of variables necessary for engineering at any scale. Potential field theory is an old method that is experiencing a resurgence of interest. Potential fields are used to study quantum mechanics at the atomic scale, crack and dislocation mobility at the micro-scale, and even bulk analysis. It encompasses many problems that can be formulated using partial differential equations. These series solutions are well suited for computerized numerical approximation. Because of recent advances in computational abilities, potential field theory deserves a fresh look as a candidate for multiscale modeling and as the math that binds each level together.
\end{abstract}




\section{TABLE OF CONTENTS}

Potential Field Theory and Its Applications to Classical Mechanical Problems.......i

TTable of Contents ...........................................................................................

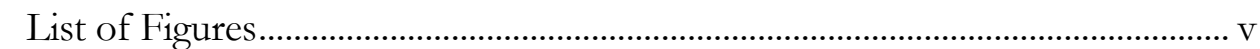

Equations .................................................................................................... vii

Acknowledgments ............................................................................. ix

Dedication ..................................................................................................................

Preface ..................................................................................................................... xi

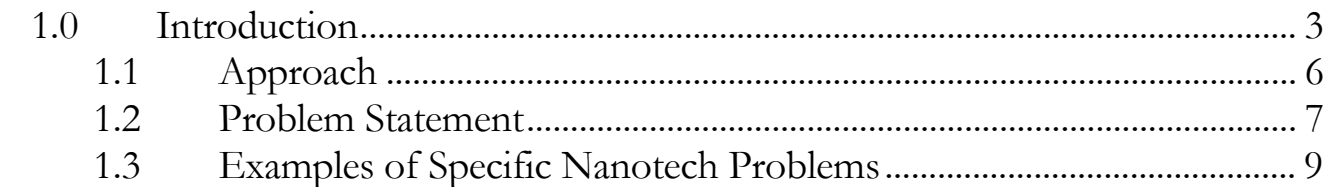

$2.0 \quad$ Literature Review of Strength .................................................................. 11

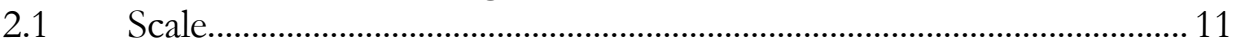

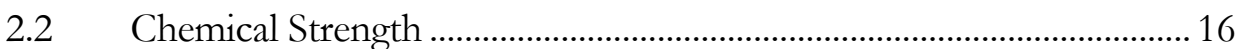

$2.3 \quad$ Measures of Strength …................................................................. 23

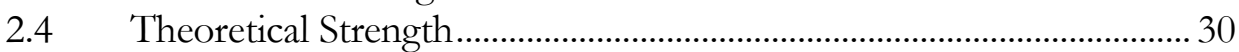

2.5 Temperature Dependence .................................................................... 33

2.6 Time Dependence ................................................................... 35

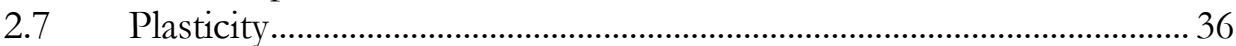

$2.8 \quad$ Ductile vs. Brittle ............................................................................ 37

2.9 Hardening Mechanisms-History Effects ......................................... 39

2.10 Hydrostatic Pressure .......................................................................... 40

$2.11 \quad$ Grain Size .......................................................................................... 41

$2.12 \quad$ Vibration and Impact............................................................................. 46

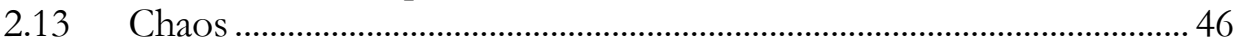

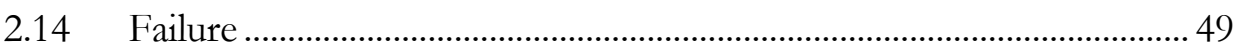

2.15 Conclusions about Strength …..................................................... 50

$2.16 \quad$ Further References ............................................................................. 51

$3.0 \quad$ Literature review and Derivation of Elasticity ....................................... 52

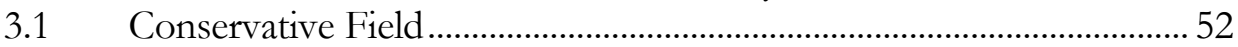

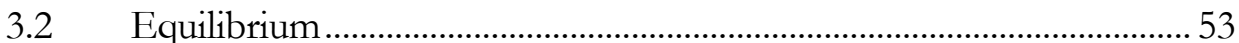

3.3 Elastic Equations Using Isotropic Conditions.................................. 62

3.4 Mathematical Terms and Theorems ...............................................6. 69

3.5 Nonlinear Formulation, Chaos, and Stability ..................................... 76

3.6 Derivation References ................................................................... 83

$4.0 \quad$ Potential Field Theory Applied to Mechanics............................................ 84

$4.1 \quad$ Method of Solution ....................................................................... 87

$4.2 \quad$ Spherical Harmonics and Legendre Polynomials.............................. 95

$4.3 \quad$ Photoelastic Fields.................................................................................. 97 


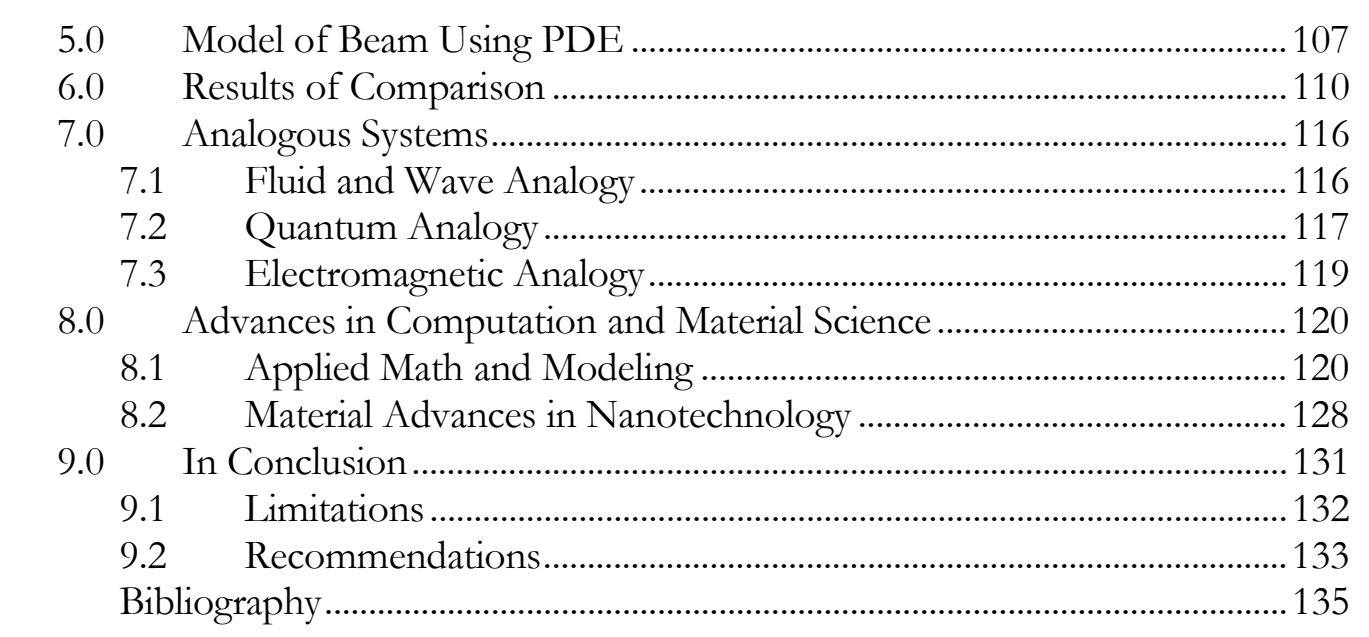




\section{LIST OF FIGURES}

Figure 2-1. Diagram of the relationships between properties and scale dependence as

concluded from the literature ......................................................................................... 12

Figure 2-2. Relationships between scales, models, and measuring techniques [112] .............. 14

\begin{tabular}{|lll|}
\hline Figure 2-3. At a constant strain rate, yield stress increases as sample size decreases [23] & $\ldots . . . .15$ \\
\hline
\end{tabular}

Figure 2-4. At constant size, 1384 atoms $7.8 \mathrm{~nm} \times 4.0 \mathrm{~nm}$, yield stress increases with

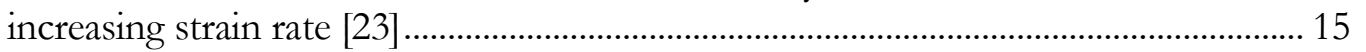

Figure 2-5. Yield stress by adiabatic modulus compared to specimen size [23] ................... 16

Figure 2-6. Bond repulsion-attraction model, potential energy vs. atomic radius [131]........ 17

Figure 2-7. Diagram of the bonding triangle from Gilman [53] ........................................... 18

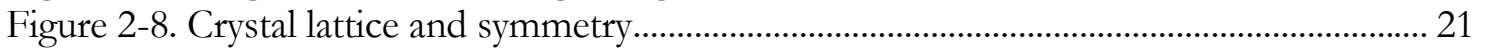

Figure 2-9. Relative size of defects [124] ....................................................................... 22

Figure 2-10. Young's Modulus vs. Atomic Number [http://www.webelements.com ]....... 25

Figure 2-11. Velocity of sound vs. Atomic Number [http://www.webelements.com ] ......... 25

Figure 2-12. Poisson's ratio vs. Atomic Number [http:// www.webelements.com ]............... 26

Figure 2-13. Bulk modulus vs. Atomic Number [http://www.webelements.com ]............... 27

Figure 2-14. Rigidity Modulus vs. Atomic Number [http://www.webelements.com ] .......... 28

Figure 2-15. Hardness in Moh Scale vs. Atomic Number, approximately logarithmic

[http://www.webelements.com ] ............................................................................ 29

Figure 2-16. Comparison of relative hardness-Brinnell, Rockwell, and Moh scales-with

material samples [127].......................................................................................... 29

Figure 2-17. Diagram of the effect of non-isothermal conditions when a load is applied

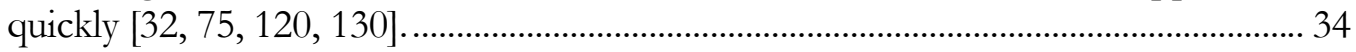

Figure 2-18. Typical Photoelastic results of the stress transmission experiment through a cohesionless material [108] ...................................................................................... 45

Figure 2-19. Superimposed results from 10 experiments with shifted grain alignment

demonstrating probability density of stress distribution. [108] ................................ 45

Figure 3-1. Diagram of the atomic bonds in a cubic isotropic crystal ....................................... 60

Figure 3-2. Dalley's table of constants and their conversion [37] ........................................... 62

Figure 3-3. Figures of beam in bending from Airy's original treatise on stress functions.

The curves are drawn from tables also included in the paper. [12]............................. 72

Figure 4-1. Illustration of 11 coordinate systems for solving partial differential equations

[90] ........................................................................................................................ 86

Figure 4-2. Electrostatic potential derived using potential fields and plotted in Matlab PDE

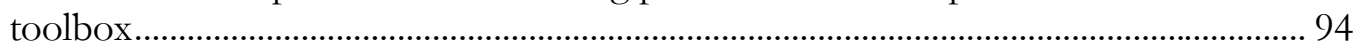

Figure 4-3. Photoelastic response at a crack tip [100]......................................................... 98

Figure 4-4. Photoelastic experiment and VIP simulation of crack tip compared with dipole

(right) generated with Equipotential 3.1 software [44] ................................................ 99

igure 4-5. Thamm's hand drawn stress fields for component design, beam in bending

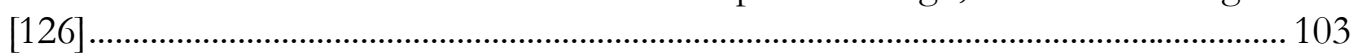

Figure 4-6. Thamm's hand drawn stress fields for engine component design [126] ............ 104

Figure 4-7. Diagram of the comparison between potential theory and photoelastic theory 105 
Figure 6-1. Absolute displacement of a beam in tension ........................................................... 110

Figure 6-2. X-displacement of a beam in tension ............................................................... 111

Figure 6-3. Von Mises Stress of a beam in tension .............................................................. 112

Figure 6-4. First principal stress of a beam in tension ............................................................. 113

Figure 6-5. Second principal stress of a beam in tension .................................................. 114

Figure 6-6. X-displacement of alternative boundary conditions for a beam in tension ........ 115

Figure 7-1. Worthington jets produced by spherical beads of sand [129]...........................117

Figure 8-1. Figures from microscale grain simulation. Note: bottom right graph of the rate

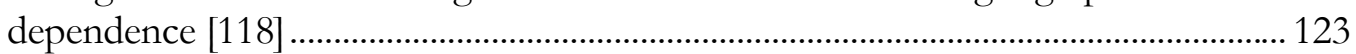

Figure 8-2. Bader's atomic simulations using quantum and functional theory for chemistry

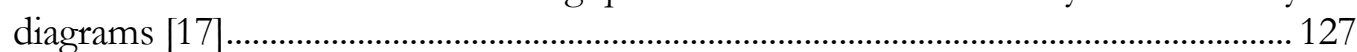

Figure 8-3. Quantum corral nanotech component [112] .......................................................... 129

Figure 8-4. Carbon nanotube with benzene teeth gear, nanotech component [112]........... 129 


\section{EQUATIONS}

Equation 2-1 Relationship between sound and stiffness ..................................................... 24

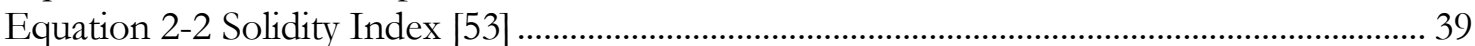

Equation 2-3 Hall-Petch Relation............................................................................................. 41

Equation 3-1 Conservation of Energy ................................................................................ 52

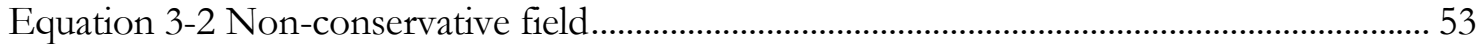

Equation 3-3 Conservative field.............................................................................................. 53

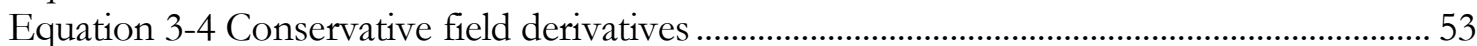

Equation 3-5 Equilibrium stress fields.................................................................................. 54

Equation 3-6 Summation of moments .............................................................................. 54

Equation 3-7 Body force fields ............................................................................................. 55

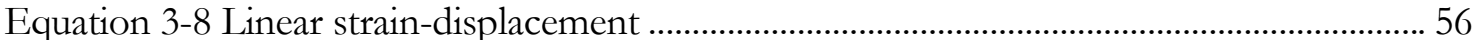

Equation 3-9 Non-linear strain-displacement ............................................................ 56

Equation 3-10 Linear rotation-displacement.................................................................... 57

Equation 3-11 Non-linear rotation-displacement................................................................... 57

Equation 3-12 Non-linear strain to linear strain and rotation [94] ........................................ 57

Equation 3-13 Stress-stiffness and strain-compliance relationships [53] ............................... 59

Equation 3-14 Triclinic elastic stiffness matrix [53] .............................................................5 59

Equation 3-15 Cubic elastic stiffness matrix..................................................................60

Equation 3-16 Anisotropy coefficient [53] ...........................................................................61

Equation 3-17 Strain - Stress relations (linear) ..................................................................6. 63

Equation 3-18 Displacement - stress relations (linear) …..................................................63

Equation 3-19 Linear, isotropic stress - strain relations [37] ...............................................6. 64

Equation 3-20 Linear, isotropic Stress - Displacement relations.......................................64

Equation 3-21 Displacement equilibrium relations [37] ...................................................6. 65

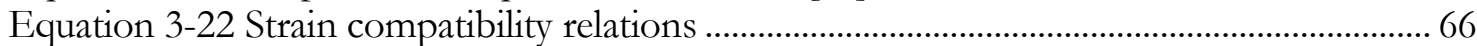

Equation 3-23 Stress equations of compatibility [37] ............................................................6. 67

Equation 3-24 Laplace's equation in two-dimensions .........................................................6. 67

Equation 3-25 Potential field equations in three and two-dimensions [75] ..........................6.68

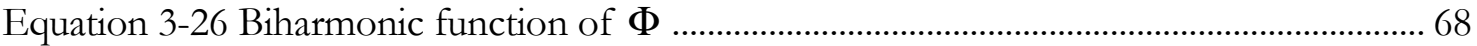

Equation 3-27 Curl [143] ......................................................................................................6. 69

Equation 3-28 Laplace's equation .........................................................................................70

Equation 3-29 Green's theorem [143] .................................................................................. 73

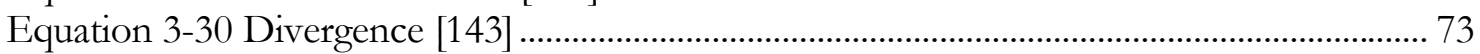

Equation 3-31 Flux [143] ...................................................................................................... 74

Equation 3-32 Stoke's theorem [143] ........................................................................... 74

Equation 3-33 The Cauchy-Riemann condition [143] ................................................... 74

Equation 4-1 Separable potential function in Two-dimensional .......................................... 88

Equation 4-2 Partial differential equations............................................................................ 88

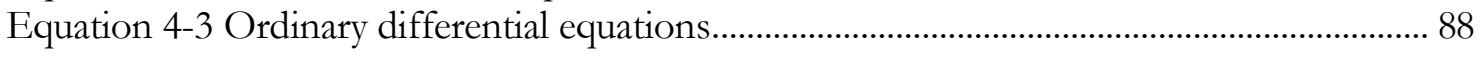




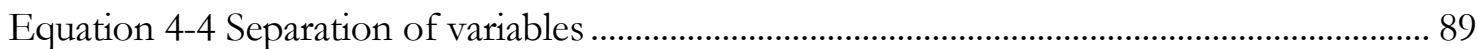

Equation 4-5 Solutions to two-dimensional separable PDE ..................................................... 89

Equation 4-6 Equivalent exponential solutions to two-dimensional .................................... 90

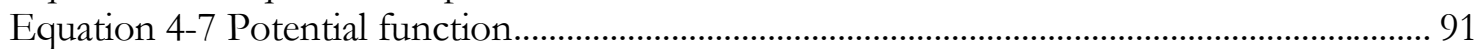

Equation 4-8 Applying boundary conditions to two-dimensional equations yield B and $\lambda .91$

Equation 4-9 Potential function................................................................................... 92

Equation 4-10 Series solution to boundary conditions ............................................................ 92

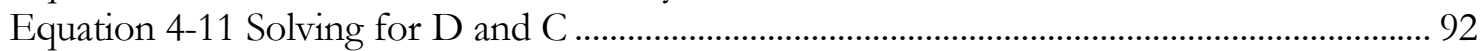

Equation 4-12 Final form of potential function ....................................................................... 93

Equation 4-13 General solution to axially symmetric separable equations in spherical

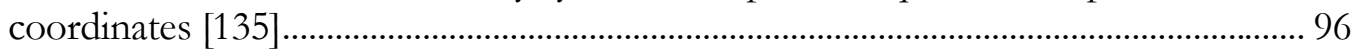

Equation 4-14 Recursive formula and first three Legendre polynomials [135] ...................... 96

Equation 4-15 Photoelastic stress extraction equations ................................................................ 100 Equation 5-1 Classical mechanical equations for a beam in tension with assigned constants

and calculated values ................................................................................................... 107

Equation 7-1. Analogous equations of potential fields [53].............................................118 


\section{ACKNOWLEDGMENTS}

The author wishes to express sincere appreciation to Jeffrey R.X. Auld for support, editing, and enlightened arguments during this project. In addition, a special thanks is reserved for Professor James Smith for his support and encouragement, and for the opportunity to follow my muse. Thanks also to the professors at West Virginia University, especially Dr. Bruce Kang, for their valuable input in aiding the scientific development of this manuscript. 


\section{DEDICATION}

This manuscript is dedicated to Mr. and Mrs. John Francis Stillwagon without who's support, confidence, and love, this undertaking would never have been possible. Thank you. 
PREFACE

\section{The Blind Men and the Elephant John Godfrey Saxe (1816-1887)}

It was six men of Indostan

To learning much inclined, Who went to see the Elephant (Though all of them were blind), That each by observation Might satisfy his mind.
The Fourth reached out an eager hand, And felt about the knee "What most this wondrous beast is like Is mighty plain," quoth he:

"'Tis clear enough the Elephant Is very like a TREE!"
The First approached the Elephant, And happening to fall Against his broad and sturdy side, At once began to bawl: "God bless me! but the Elephant Is very like a WALL!"

The Second, feeling of the tusk, Cried, "Ho, what have we here, So very round and smooth and sharp?

To me 'tis mighty clear This wonder of an Elephant Is very like a SPEAR!"

The Third approached the animal, And happening to take

The squirming trunk within his hands,

Thus boldly up and spake:

"I see," quoth he, "the Elephant Is very like a SNAKE!"
The Fifth, who chanced to touch the ear, Said: "E'en the blindest man

Can tell what this resembles most; Deny the fact who can, This marvel of an Elephant Is very like a FAN!"

The Sixth no sooner had begun About the beast to grope, Than seizing on the swinging tail That fell within his scope, "I see," quoth he, "the Elephant Is very like a ROPE!"

And so these men of Indostan Disputed loud and long, Each in his own opinion Exceeding stiff and strong, Though each was partly in the right, And all were in the wrong! 


\section{Reference for U nderstanding this Text}

Figures come directly from references sited in the caption unless they are diagrams, which have been prepared by the author. The original captions have been retained for context but their caption numbers have been removed to avoid confusion with this documents numbering system. Diagrams are original illustrations by the author which have references sited if they are adaptations of another author's work.

References are not abbreviated in any way to facilitate ease in locating rare and unfamiliar journals. References are sited in brackets, like [205], where the number is linked to the reference number in alphabetical order by the primary author's last name. Headings followed by reference numbers in brackets, indicate references of interest to the topic for further review.

Equations are numbered by the chapter number and indexed 1, 2, 3, etc. in each chapter. Because many equations are reproduced from sources outside of the field of engineering, in many instances a symbol's meaning is not familiar and not consistent, between math, physics, or chemistry derivations. Therefore, an effort has been made to define the symbols with the equation that introduces the symbol along with an explanation and engineering comparison, if necessary; and unrelated symbol duplication is avoided to prevent confusion. When specific equations have been reproduced from a single source, the reference is sited in the caption. However, many equations are general derivations that are found in many texts; these general equations can be found in many reference books but are only referenced in the text to sources from the bibliography that contain 
these general expressions. The original source and initial derivation is not sited or used in this document. A table of equations with captions and page numbers is provided for easy reference.

Each sub-heading in the chapters contains a specific contribution to the chapter as a whole, but there is no natural order to these components. This is aggravated by the fact that many headings have a synergistic relationship to one another. Because of this, the order that topics are discussed may appear arbitrary and many headings and figures are cross-referenced before they are formally introduced. The Microsoft Word document is linked to jump forwards and backwards to the cross-referenced sections, demonstrating how everything is linked together and interdependent. The navigation is like a web page to avoid repetition and circular arguments. To facilitate and encourage correct navigation of this text, page numbers and section headings for cross-referenced items are included. This web page style linkage allows parallels to be made that otherwise would be lost, and it emphasizes the equal weight given to each component or sub-heading. 


\section{Introduction}

Potential field theory is an old method that is experiencing a resurgence of interest due to advances in computational ability and the method's flexibility in modeling disparate phenomenon. Potential field theory is the mathematical study of partial differential equations of which Laplace's equation is particularly important to stress and strain analysis. Advances made in numerous scientific fields in the modeling and solving of partial differential equations lead to results that are applicable to Classical Mechanical engineering problems.

Strength is investigated in Section 2.0, and the elephant of the preface is a fitting symbol of strength. The men represent the sciences, each having their own theories and definitions yet blind and argumentative towards the others. This Section covers all aspects of strength using the methods gleaned from their observations for the purposes of engineering. To understand the entire elephant requires cross-discipline investigation and a synthesis of their respective models.

The changes in material properties has been a serious handicap for mechanical engineers wanting to contribute to nano-tech because many of the assumptions based on bulk properties are invalidated at micro and nano scale, and many phenomena go contrary to (bulk) experience [112]. An entirely new set of tables for engineering materials has to be created for different scales, because the bulk tests are difficult if not impossible to extrapolate to smaller scales [12]. This is an incredible amount of work and testing at a tremendous expense because of the sensitive equipment necessary to observe small-scale 
response. Just one material will have to be tested at several scales to produce a curve representing the effect of scale on strength (and changes in material properties), and this needs to be repeated for all engineering materials. To better understand strength and the strength of materials, many well-known methods and analyses are revisited in Section 2.0 in an attempt to tie together phenomena that are inconsistent with bulk assumptions about strength. For instance, the increase in strength witnessed in materials as the scale is reduced (from millimeter to micro- and to nano-) [See Figure 2-3, Eigure 2-4, Figure 2-5]

It has become important to investigate what engineering principles do apply at small scales. The founding fathers of this science were indistinguishable from physicists, and most of their principles were formulated without a dependence on scale as is discussed in Section 2.0 and 3.0. The assumptions made in the study of elasticity are noted and their limitations are explored. The fields that are approximately solved by bulk elastic models are exactly solved by potential field functions as illustrated in Dally, [37, 30]. These functions produce photoelastic-like graphs and are the recognized exact solutions to stress and strain problems [See Figure 3-3, Figure 4-3, Figure 4-4, Figure 8-1, and references 78,00 . Analytical solutions for these problems are very difficult and when solvable they involve infinite series, spherical harmonics (Legendre polynomialsspherical coordinates), and Bessel functions (cylindrical coordinates). The stress fields produced in this manner are scale invariant, (the material constants of strength vary with scale), and the computer capabilities to numerically solve and graph these functions are now available $2,20,23,31,73,102,121$. 
and the mathematical approach to solving Section 4.0 outlines potential field theory, its use in engineering, The physical or experimental expression of the potential field is photoelasticity. Graphical isostatic stress fields generated using potential theory are identical to the isochromatic fringes produced in photoelastic experiments. The stress function also has an electromagnetic analogy. James Clerk Maxwell introduced both electromagnetic equations and experimental photoelasticity in the late eighteen hundreds 83. 84, 86. Static electromagnetic field equations must satisfy Laplace's equation Equation 3-28, yielding electromagnetic fields similar to mechanical stress fields [See Section 7.3 Electromagnetic Analogy].

Applying potential field theory to the simplest classical mechanical problem, a beam in tension, produces interesting results demonstrated in Sections 5.0 and 6.0. The results justify bulk assumptions meant to simplify the model and are mathematical realizations of photoelastic experiments. Saint-Venant's principle is well-modeled in the results.

The final analogy to quantum physics is interesting as well [See Section 7.2]. Solutions to Schrödinger's Wave function can be expressed as Spherical Harmonics and Legendre Polynomials, which have been used to demonstrate the shape of the shells where electrons are most likely to be found. It is fitting that electromagnetic and quantum solutions should find their way into the explanation of strength, because it is just these properties at work in the atomic scale which give rise to the bulk behavior of materials from the strength of bonds between atoms and molecules $228,37,42,43,53,65,66,70$, 73, $75,83,84,87,88,93,96,98,102,103,104,106,112,114,119,128,127,130,133$, 
138. Several scientific groups are modeling these forces to produce models of atomic interaction that can be scaled upwards to generate bulk properties [See Section 8.2 Material Advances in Nanotechnology

This entire work is a literature review that crosses many discipline boundaries, demonstrating and acknowledging the need for cross-discipline teams to work on this subject. Potential field theory is a powerful tool that is applied at every scale and in many field applications. The development of material models from the atomic level up to bulk structures is making use of this flexibility. To meet the needs of nanotechnology engineers are needed to design at smaller scales.

All work presented in this evaluation is the labor of great scientists of the past that has been continued by many notable scientists of today and references have been liberally sited.

\section{Approach}

- Review of strength, the variables, and how strength is effected by scale, Section 2.0 Literature Review of Strength

- Review of classical mechanics derivations to observe where scale invariance is lost, Section 3.0 iterature review and Derivation of Elasticity

- Introduce Potential Field Theory as the math that binds the scales and variables in strength, Section 4.0 Potential Field Theory Applied to Mechanics

- Model classical beam problem using Potential Fields for illustrative purposes, Section Emor! Reference source not found. Ermor! Reference source not found. 
- Comparison of Classical Theory and Potential Theory solutions, Section Error! Reference source not found. Error! Reference source not found.

- Review analogous systems that use and advance Potential Field Theory, Section 7.0 Analogous Systems

- Describe advances made in modeling and computation that make progressive work in Potential Field theory feasible, and the advances made in materials that make Potential Field theory necessary, Section 8.0 Advances in Computation and Material Science

- Define the needs of Nanotech that are keenly suited to mechanical engineering expertise, Sections 1.0 and 3.0 .

\section{Objectives:}

1. To support the use of Potential Field Theory in the modeling of mechanical problems,

2. To demonstrate the similarities between Potential Field Theory, Photoelasticity, and Electromagnetic phenomenon,

3. To provide background to the issues of strength and the loss of scale invariance,

4. To demonstrate the method of separation of variables to solve PDE's, and

5. To model a beam in tension to show the deviation from classical theory.

\subsection{Problem Statement}

As advances are made in nanotechnology, needs for design, controls and analysis, particularly thermal and mechanical stress are becoming available in this new field. Work that typically comes under the auspices of engineering is needed, but many engineers are uncomfortable designing at this level because classical or continuum mechanics does not apply and quantum mechanics is said to apply in a tangible way $2,17,20,23,32,38,39$, 
42, 43, 44, 49, 50, 51, 53, 63, 66, 69, 63, 83, 66, 102, 103, 104, 106, 107, 112, 118, 123,

133, 140]. More precisely, why are classical methods ineffective on smaller scales? What are the assumptions that lead to classical method abandonment at small scales, and what needs to be changed to allow engineers to do their job at these scales?

To answer these questions, it is necessary to understand strength, in all of its manifestations, and how changing scales affects the strength of materials. This leads directly to sets of variables necessary for engineering at any scale. A nano-engineer must understand these variables in material properties and their scaling behavior like a material scientist.

Within the derivations of classical mechanics are assumptions that limit its use to bulk engineering. What are these assumptions, where do they come into the equations, and can they be changed to extend their use to smaller scales?

Potential field theory may or may not be familiar to engineers; as a design tool, it is scale invariant. Potential fields are used to study quantum mechanics at the atomic scale, crack and dislocation mobility at the micro-scale, and even bulk analysis. However, it is rarely used for bulk calculations; because the math is formidable, and even the few analytical solutions must use numerical approximations to solve the infinite series. Potential field theory encompasses many problems that can be formulated using partial differential equations. 
Because of recent advances in computational abilities, potential field theory deserves a fresh look as a candidate for multiscale modeling and as the math that binds each level together.

To design competently at any scale, the nano-engineer:

- Must understand material properties at each scale,

- Must understand quantum and electromagnetic contributions, and

- Must understand potential fields, their boundary conditions, and their solutions.

Advances in many fields are expected to come from work in nanotech. There is an opportunity to contribute to the design, controls, and analysis of systems that is uniquely suited to mechanical engineering if the challenges can be met. With this in mind, the following pages attempt to define the needs of mechanical design at multiscale, the role of potential theory in multiscale applications, and what steps can be made to attack this challenge.

\subsection{Examples of Specific Nanotech Problems}

Many nanotechnology problems lie in the field of engineering expertise. Classical mechanics cannot solve these difficult problems; it requires a cross-discipline approach.

- The solution of cantilever beam vibrations at the microscale, while respecting nano- and microscale contributions, applies to the quartz crystal microbalance, a delicate experimental testing apparatus. [See bonds, nanotechnology, vibration/impact, $11,13,27$ 
- The solution of vibration and kinking in strings, one-dimensional problems that are very difficult to model with finite element software, applies to dislocations, nanocarbon tubes, and whiskers important to high strength applications and wiring in nano-components. [See Nonlinearity, 13, 27, 66] 


\subsection{Literature Review of Strength}

Strength is the ability to resist change and to be able to recover from stress. There are many different measures of strength, Young's modulus, yield strength, fracture toughness, hardness, and others. However, it is not one measurement that makes a material strong, but the combination of values selected to satisfy a particular purpose and withstand a particular load at a particular scale. Spider silk has a maximum tensile strength of $1.3 \mathrm{GPa}$ as compared to mild steel's $400 \mathrm{MPa}$ while carbon nanotubes boast over 100 times the tensile strength of steel [25]. Superhard materials are being developed at more than $40 \mathrm{GPa}$ with applications to coatings and thin films to replace materials that are environmentally hazardous [133.

\subsection{Scale}

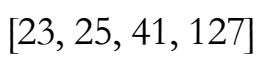

There are many levels of aggregation from atoms to large engineering structures [3], but this thesis will only be concerned with three, Bulk or macro, micro, and nano. Bulk or macro-scale properties are those that work at the level with which engineers are familiar and can be observed with the unaided eye. Micro-scale properties can be observed with a microscope and are the link to nano-scale. At the molecular and atomic level, quantum mechanics are active and are different from bulk behavior. Some properties, labeled scale independent in Eigure 2-1, are apparent at all scales, though the effects may be different at each. Some of the complexities of scale are illustrated in Figure 2-1 and are discussed throughout this chapter. 


\section{Bulk or Macro Properties}

Size Dependency

Geometry

Ductile-Brittle transition

Measures of Strength - Dimensional Ratios see Constants, Bulk Moduli-Poisson's Ratio, Young's modulus, Shear modulus, Bulk modulus, Fracture toughness, Resilience

Temperature

Density

\section{Micro Properties}

\section{Grain Size}

Defects

Dislocations

Plasticity similarities to fluid flow

Density Fluctuations

\section{Scale Independent Properties}

\section{Nano Properties}

Atomic Bonds, binding energy,

Defects vs. Purity

Quantum effects

Hopping

Phonons

Plasmons

Photons

Electron wind (damping)

Charge Density

\section{Theoretical Strength}

Isotropy or Anisotropy

Geometric- Notches, Cracks

Crystallography and Geometry (Anisotropy)

Temperature Dependence- Thermal Expansion

Time Dependence- History Effects

Force Distribution

- Hydrostatic Pressure

- Vibration and Impact

Surface and Atmospheric Conditions

Scale or Gauge Dependency

\section{Figure 2-1. Diagram of the relationships between properties and scale dependence as concluded from the literature}


A nanocrystalline material is defined as having grain sizes Grain Size less than 100 nanometers or one millionth of a millimeter 122. Figure 2-2 shows the spatial and temporal scales and where different methods of analysis and experimentation occur with respect to characteristic lengths and time scales.

Dependence on sample size is one of the most important issues to multiscale engineering. Holding the strain rate constant, the yield stress is a monotonically decreasing function of sample size, Eigure 2-3 23]. Holding the sample size constant and increasing the strain rate also produces an increase in yield strength, Figure 2-4[23]. This variable behavior in a material constant becomes a major issue at small scales, and defects, Section 2.2.3, are the main cause of this behavior.

One of the most interesting characteristics of scale is that materials tend to be stronger at smaller scales [See Figure 2-3 and Figure 2-5]. Figure 2-3 and Figure 2-5 both show increased yield strength as scale is reduced. Continuum Mechanics cannot account for this phenomenon, and bulk materials are 100 times weaker than theoretical strength predicts using chemical bond strengths 141]. In Figure 2-5 there is a logarithmic relationship between yield stress divided by Young's modulus, for a normalized non-dimensional quantity, and the sample size. 


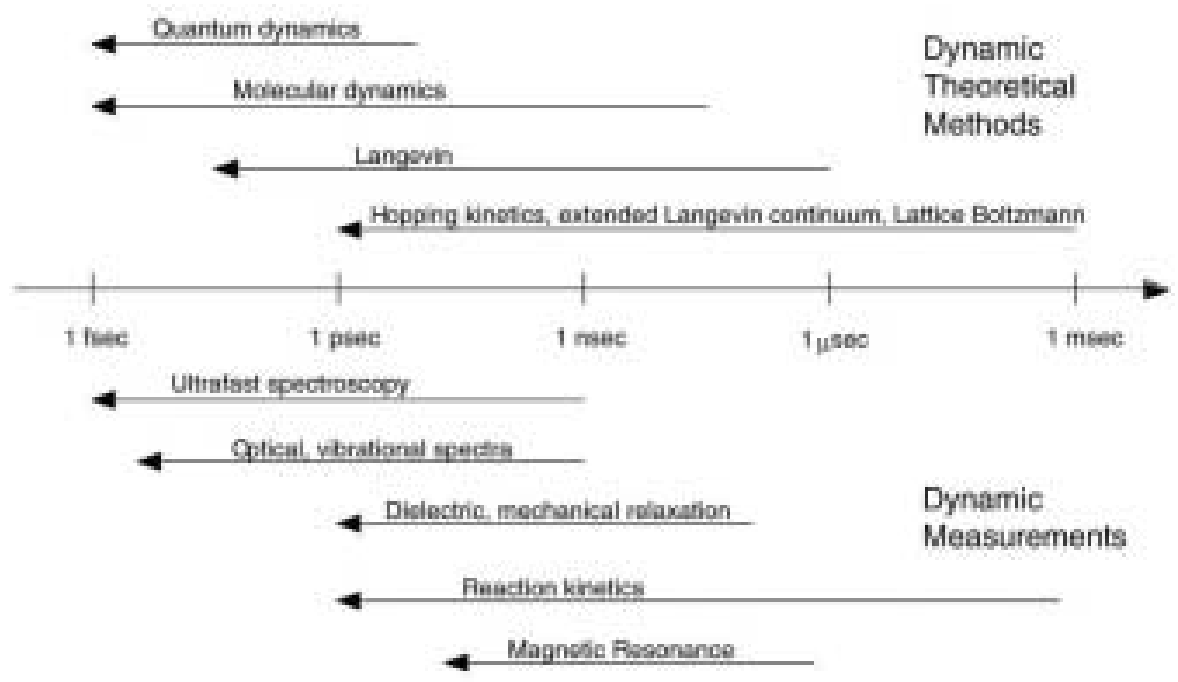

Temporal Scaling

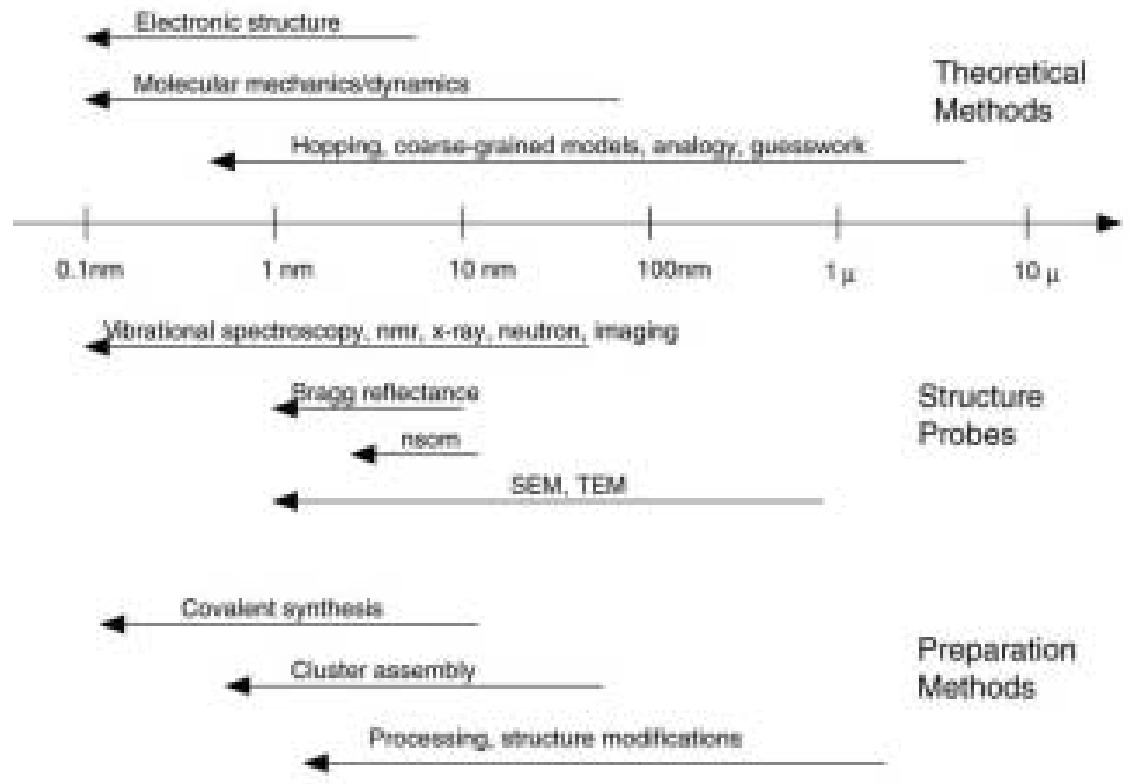

Spatial Scaling

Figure 2-2. Relationships between scales, models, and measuring techniques [112] 


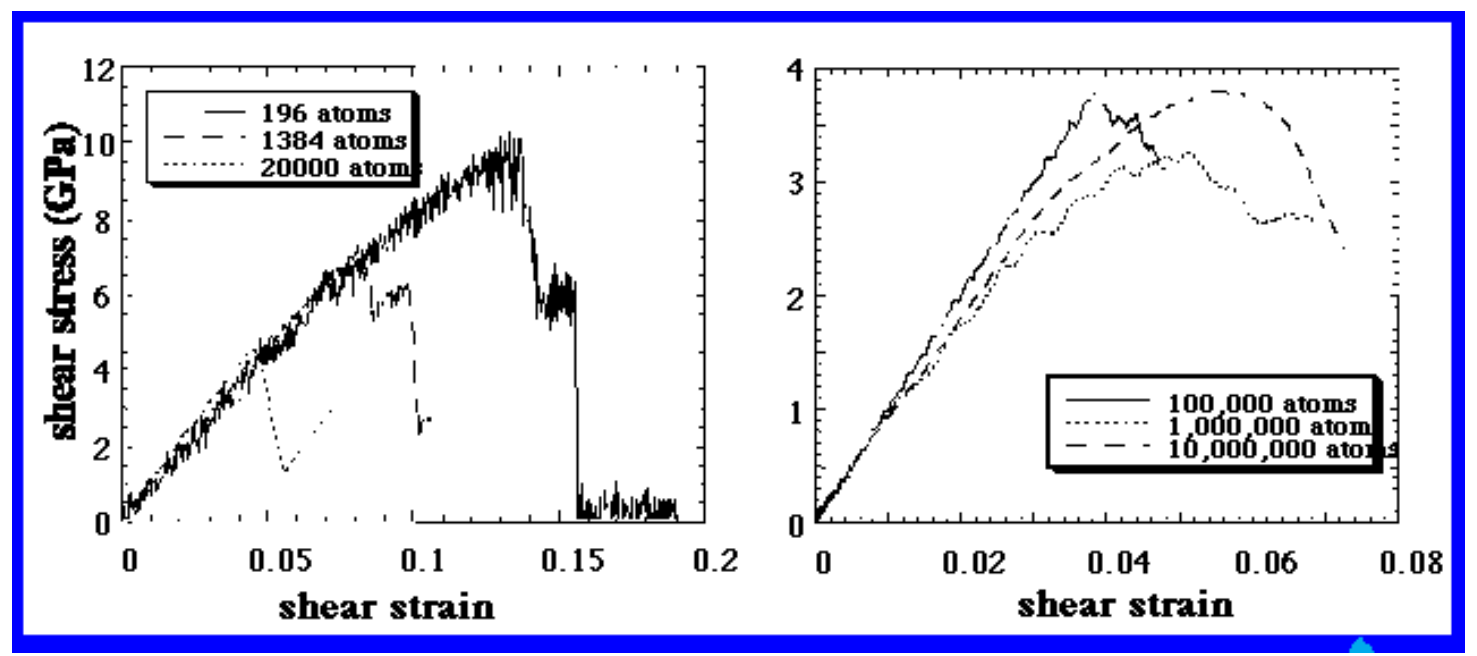

Figure 2-3. At a constant strain rate, yield stress increases as sample size decreases [23]

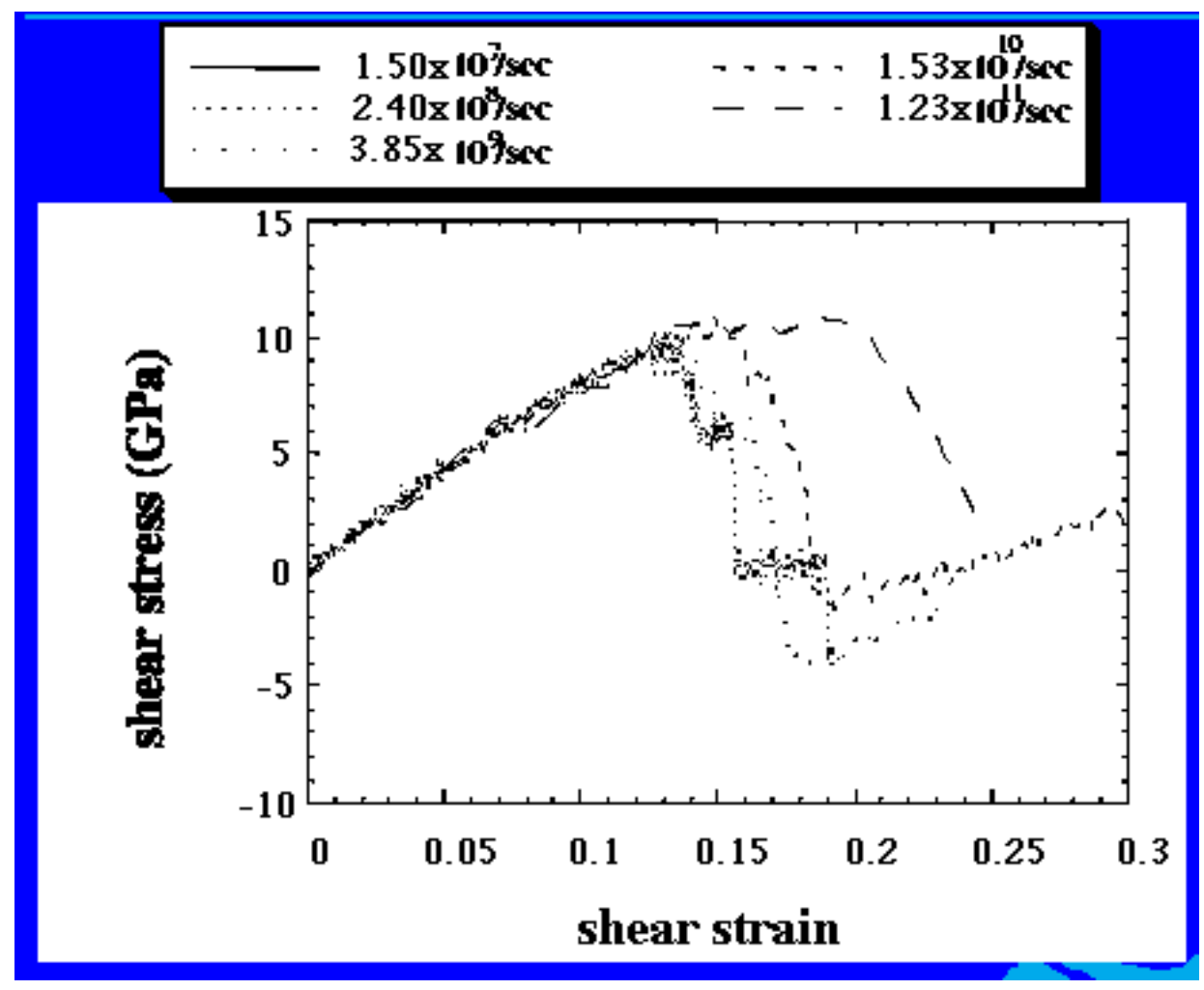

Figure 2-4. At constant size, 1384 atoms $7.8 \mathrm{~nm} \times 4.0 \mathrm{~nm}$, yield stress increases with increasing strain rate [23] 


\section{Sample Size is the Primary Determinant of Yield Stress}

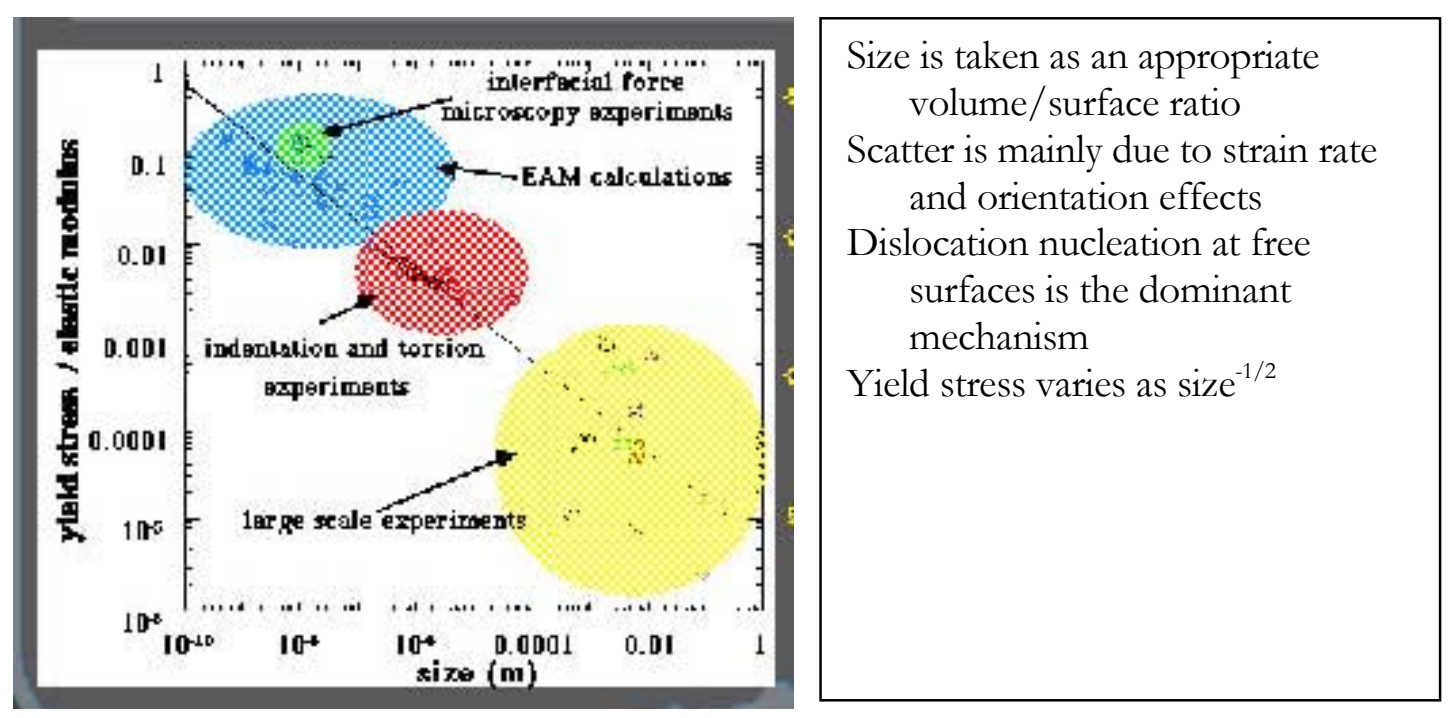

Figure 2-5. Yield stress by adiabatic modulus compared to specimen size [23]

\subsection{Chemical Strength}

The source of the strength of a material is linked to atomic bonds. Even before direct evidence of atoms and electrons, this theory supposed a smallest unit of matter, an atom, bound to another [75]. The attraction and repulsion between these units, graphed in Figure 2-6, determined whether a material was a solid, liquid, or gas and basic properties like strength. At the smallest level, bond strength is the first determining factor to strength. Figure 2-6 is a qualitative model of bond repulsion-attraction. The attraction is electromagnetic, and the repulsion is a quantum effect from the Pauli exclusion principle, which prevents electrons from having the same quantum numbers and in effect overlapping [104]. 


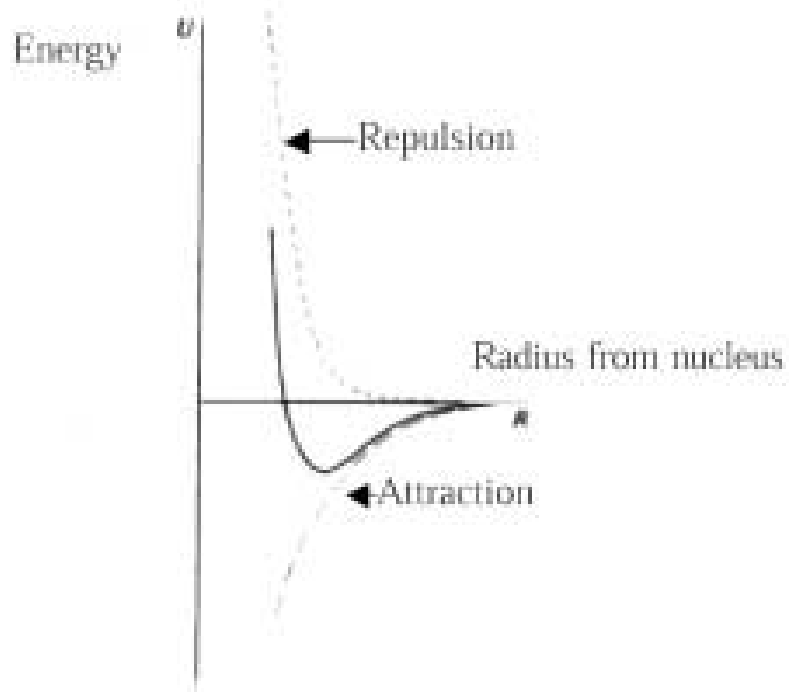

Figure 2-6. Bond repulsion-attraction model, potential enengy vs. atomic radius [131]

Atoms come in over 100 shapes and sizes as witnessed in the periodic table, and, according to strict rules, they can combine to produce compounds. The electronic configuration, especially of the outer shell, determines what arrangement and which bonding is available and favorable. Electronic configuration, shells (s,p,d), spins, and orbital shapes are understood/modeled using quantum mechanics and specifically, Schrödinger's wave equation. Spurred by nanotechnology, new materials, new applications and new methods for producing designer materials are continually being explored because of the wealth of atomic building blocks. 


\subsubsection{Atomic Bonding}

$11,15,39,3,125$

Primary bonding comes in three principle varieties with smooth transitions between the poles or vertices, see Figure 2-7, the bonding triangle from Gilman [33]. Within the triangle lie mixtures of the pure bonding achieved at the vertices. The bond strength varies from 60 to $300 \mathrm{kcal} / \mathrm{mole}[127$, Secondary bonding or Van der Waals forces are less than 10 $\mathrm{kcal} / \mathrm{mole}$, and these are due to dipole interactions and include hydrogen bonding and London dispersion forces [127].

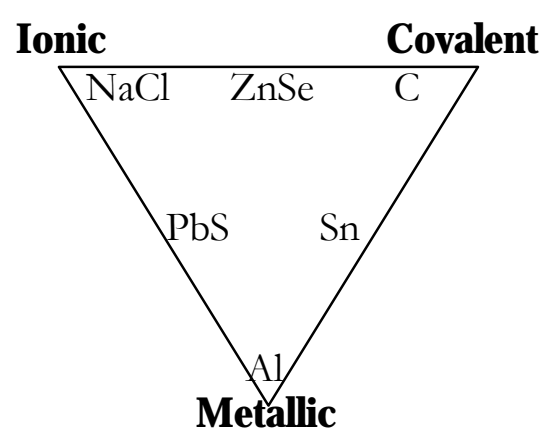

Figure 2-7. Diagram of the bonding triangle from Gilman [53]

Covalent bonds share at least one pair of electrons between atoms, trying to achieve a full outer shell in each of the contributors. Covalently bonded materials are intrinsically harder than metals or ionic solids, and they resist plastic deformation [53]. They are especially resistant to shear, and the shear moduli are often larger than the bulk moduli [33]. The covalent bond was measured in a bond-breaking experiment that found it to be 600 times stronger than non-specific bonding and 10 times stronger than hydrogen bonding [11]. It is 
in general the strongest type of bond; it has high melting temperatures and a more open crystal structure [28].

Ionic bonds occur between metallic and non-metallic elements, where the valence electron is completely given to the non-metal creating a cation (negative charge) and anion (positive) pair. The ions gain stability by having full outer shells and a charge from the gain or loss of electrons. This electromagnetic charge is what binds the compound. It is found in salts and ceramics and has a densely packed crystal structure.

Metallic bonds metallic bonds have a surplus of electrons, which congregate in an electron cloud giving metallic compounds their characteristic ability to conduct electricity [119].

Molecular bonds are the weakest, and are present in polymers, rubber, noble gases, and between layers of materials, like graphite or mica [53. They are secondary bonding forces that include dipole interaction and hydrogen bonding. Neutral atoms in close proximity induce dipoles in the atoms resulting in London forces [88]. These forces are responsible for bonding between fibers into bundles and between lamellae to form flakes, as in graphite. Hydrogen bonding is also an induced dipole and gives water its unusual and lifesupporting properties [9, 104, 135. It is present and important to many organic compounds, like proteins, as well as polymers [26, 41]

The strongest compounds tend to be those with the highest melting points and heats of formation [53]. 


\subsubsection{Crystallography-Geometry}

$26,28,38,40,44,63,65,70,106,140$

Crystals have periodic structure as illustrated in Figure 2-8. Material constants [See Section 3.2.4 Constants on page 61] can vary in different directions with respect to the lattice; this is an anisotropic structure [See Equation 3-16 Anisotropy coefficient]. Isotropic crystal properties are independent of direction. Homogeneous materials may also display isotropic values because of a homogenous mix of crystal grain orientation, for example steel. Crystal shape and symmetry bare an important relationship to the number of constants needed to describe a material's properties or response [See Section 3.2.4 Constants on page 61 and References 6, 28, 42, 63, 65, 106, 118, 140.

Amorphous materials or glasses have chaotic structures with random patterns or nonperiodic bonding as opposed to crystalline structures. The lack of periodicity impedes dislocation mobility and the materials are brittle and may cry (acoustic emission) during yielding [53]. Amorphous literally means without form in contrast with crystalline, which has a geometric form. Glass and ceramics are the most common amorphous materials. 
CUBIC

$\mathrm{a}=\mathrm{b}=\mathrm{c}$

$\alpha=\beta=\gamma=90^{\circ}$
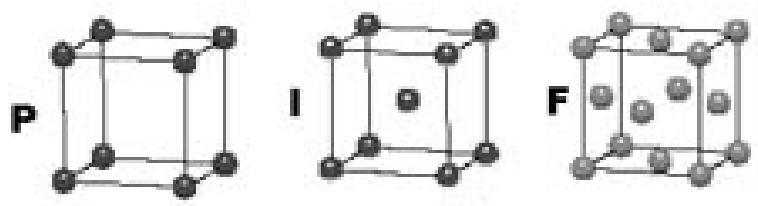

TETRAGONAL

$\mathrm{a}=\mathrm{b} \neq \mathrm{c}$

$\alpha=\beta=\gamma=90^{\circ}$
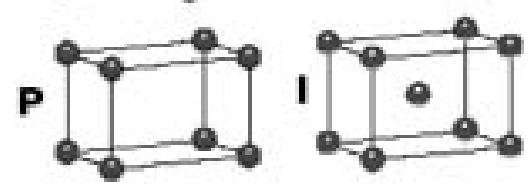

ORTHORHOMBIC

$a * b * c$

$\alpha=\beta=\gamma=90^{\circ}$
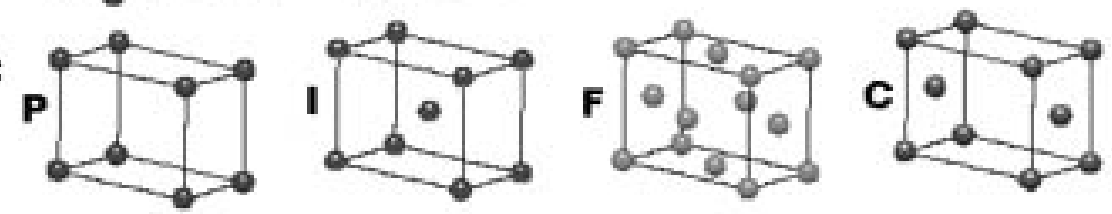

HEXAGONAL

$\mathrm{a}=\mathrm{b} * \mathrm{c}$

$\alpha=\beta=90^{\circ}$

$\gamma=120^{\circ}$

\section{MONOCLINIC}

$\mathrm{a} \neq \mathrm{b} \neq \mathrm{c}$

$\alpha=\gamma=90^{\circ}$

$\beta \neq 120^{\circ}$

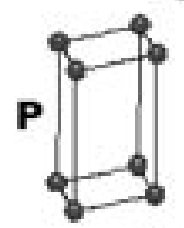

TRIGONAL

$\mathrm{a}=\mathrm{b}=\mathrm{c}$

$\alpha=\beta=\gamma \neq 90^{\circ}$

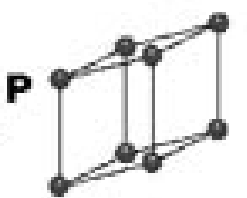

TRICLINIC

$a \neq b \neq c$

$\alpha \neq \beta \neq \gamma \neq 90^{\circ}$
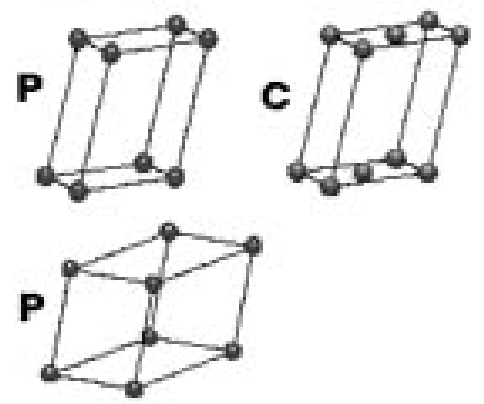

4 Types of Unit Cell
$\mathbf{P}=$ Primitive
$\mathbf{I}=$ Body-Centred
$\mathbf{F}=$ Face-Centred
$\mathrm{C}=$ Side-Centred
+
7 Crystal Classes
$\rightarrow \mathbf{1 4}$ Bravais Lattices

Figure 2-8. Crystal lattice and symmetry

[http:/ / www.chem..ox.ac.uk/ icl/ heyes/ structure_of_solids/ Lacture1/ Lec1.html ]

\subsubsection{Defects}

42 53]

Any defect in a material results in a stress concentration [6, which significantly lowers the ideal strength ${ }^{1}$ of a material [66, 23] Defects include, but are not limited to:

\footnotetext{
${ }^{1}$ Ideal, Theoretical, and True Strength are terms used to describe the maximum strength a material can achieve based on the chemical bonds, temperature, and a failure theory assuming the method of dissolution or separation of the material [See Theoretical Strength on page 30].
} 
Thermal vibrations or phonons, excitons, color centers, stacking faults, interstitials, vacancies, impurities, micro-cracks, line defects, slip planes, twinning, and environmental contaminants (on the surface boundary) $28,38,48,53,65,66$, 93, 106, 140.

Figure 2-9 displays the relative size of different types of defects. Point defects include vacancies-missing atoms, interstitials-extra atoms, and substitutional-extra foreign atoms. Point defects can diffuse, either through volume diffusion or through grain boundary diffusion depending on available energy.

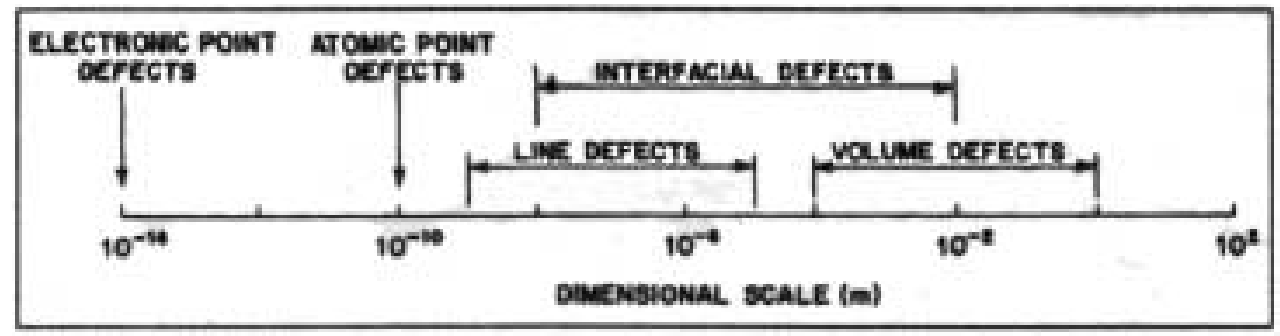

\section{Dimensional ranges of different classes of defects.}

\section{Figure 2-9. Relative size of defects [124]}

Dislocations are characterized by line defects, edge defects, or screw defects, and they move by dislocation glide on slip planes, or glide planes [53]. Dislocations can meander through a body-centered cubic metal in various directions and across planes in an entirely chaotic manner, similar to Brownian motion or random walk. They are opposed by the drag created by free electrons and by phonons [53]. The Orowan mechanism describes how dislocations cross planes causing pinning, tangling, and as a result, work-hardening [93]. Dislocation bypass by Orowan mechanism accounts for variation in strength when particles or 
obstacles are between $100-150 \mathrm{~nm}$ and shear stress is approximately $1 / 500$ of the shear modulus [53]. Line dislocations do not follow conventional statistical mechanics, but an understanding and modeling of their effects provides the critical link between atomic and

continuum scales [53, 73]. A Monte-Carlo simulation of these effects is currently being investigated by CMSN (the Computational Materials Science Network) group at Lawrence Livermore National Laboratory [73]. Other defect simulations and generation studies include $[5,42,48,50,70,73,106,112,118$.

Peierls stress is the frictional shear generated by a dislocation as it moves through the lattice, but for metals, this stress under-estimates experimental strength [15]. The Peierls-Nabarro is a mechanical model (a classical model not applicable to atomic scale) that does not give good correlation qualitatively or quantitatively, partly because the potential is continuous when, in fact, dislocation cores are singularities [53]

\subsection{Measures of Strength}

When designing a mechanical system, the strength of the constituent materials is of vital importance. This strength is either part of the initial conditions, or else the entire design hinges from the unknown parameter of strength. Factors of safety are based on it and failure theories are built around it. Determining and utilizing a material's strength is of paramount importance to engineering applications [See Section 3.2.4 Constants on page 61]. Some of the constants and ratios defined in mechanical engineering are enumerated below: 
Young's modulus (E), the resistance to tensile force, is the ratio of axial stress to axial strain under tensile loading [See Figure 2-10]. Also called stiffness, it characterizes a material's ability to withstand tensile force and is the slope of the stress-strain diagram before yield. It is related to the speed of sound through isotropic materials by equation Equation 2-1, where $\mathrm{V}$ is the speed of sound [See Eigure 2-11] and $\rho$ is the density.

$$
V=\sqrt{\frac{E}{\rho}}
$$

\section{Equation 2-1 Relationship between sound and stiffness}

Figure 2-10 is a graph of Young's modulus for the elements in the periodic chart. The atomic number identifies the element, and the periodic curve is due to outer shell electron interactions. Figure 2-11 graphs the velocity of sound propagating through the elements of the periodic chart. The velocity is related to Young's modulus through equation, Equation 2-1.

\footnotetext{
${ }^{2}$ Nabarro was a student of Orowan.
} 


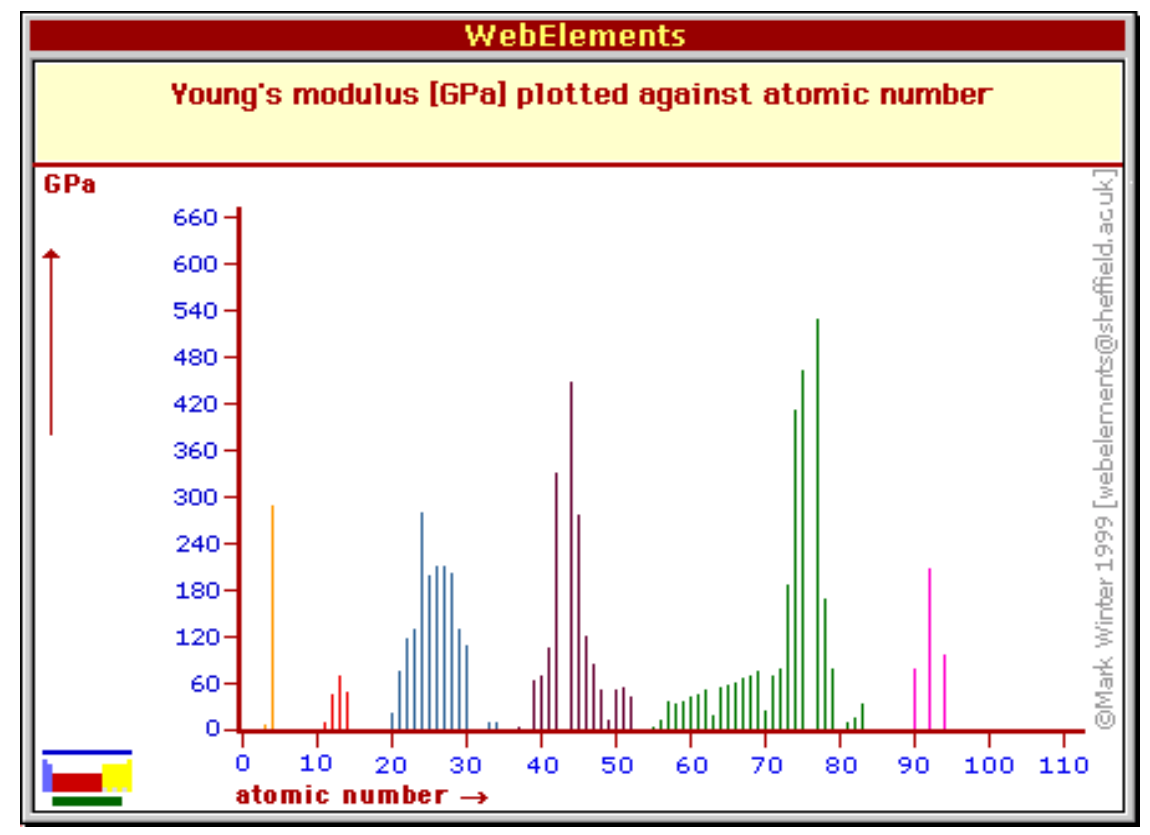

Figure 2-10. Young's Modulus vs. Atomic Number http:/ / www.webelements.com

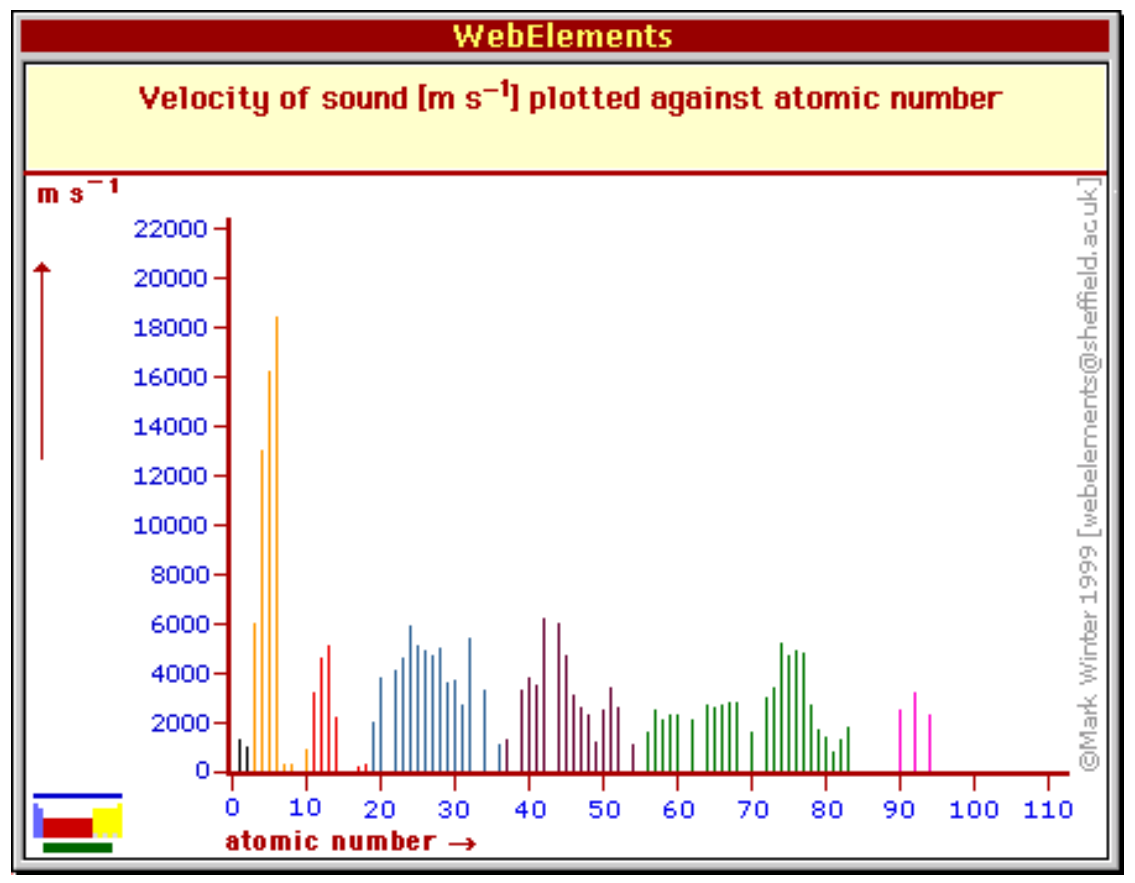

Figure 2-11. Velocity of sound vs. Atomic Number [http:/ / www.webelements.com] 
Poisson's ratio (V) is the ratio of transverse extension to longitudinal contraction [See Figure 2-12. Figure 2-12 demonstrates the elements departure from pure isotropic crystals. One constant isotropy predicts a value of 0.3 for Poisson's ratio, which is not achieved by the majority of elements [See also Figure 3-1, Equation 3-16, and Cauchy relation:]

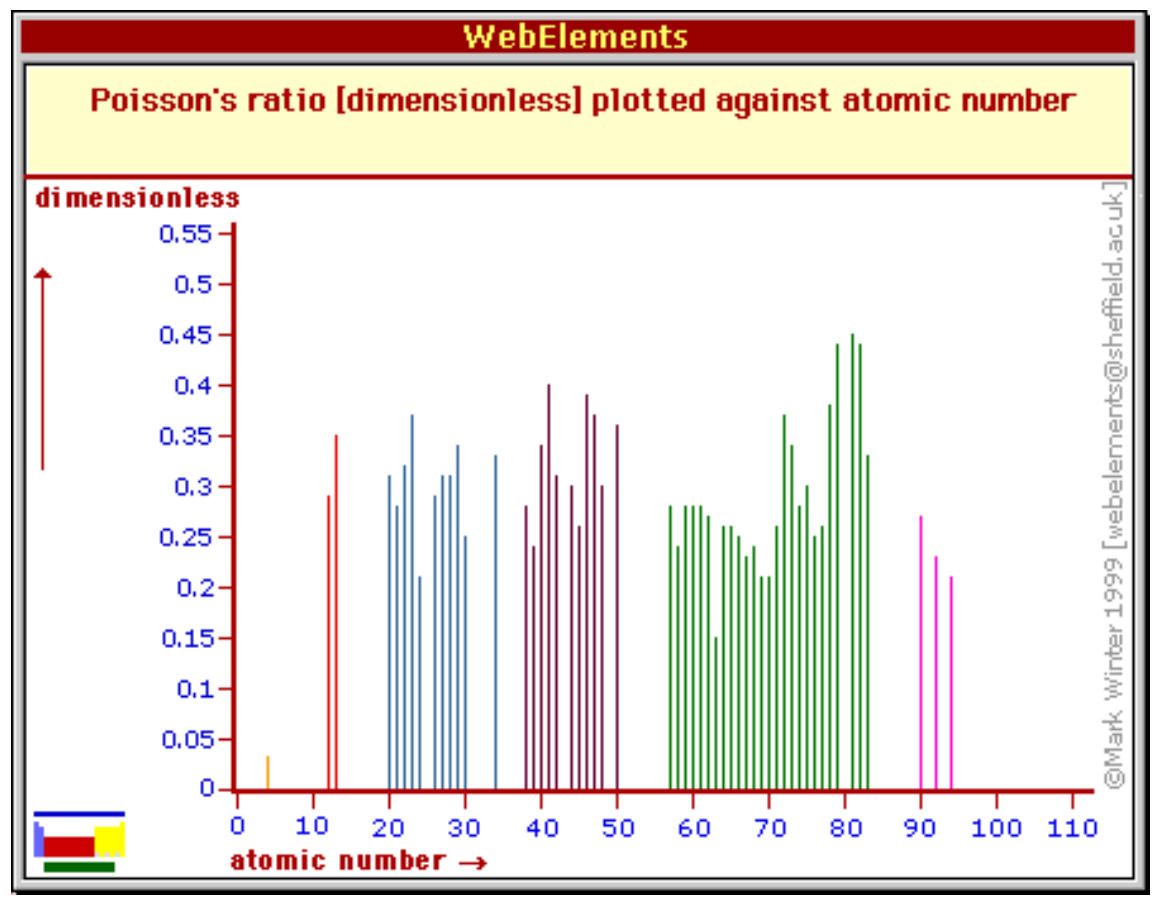

Figure 2-12. Poisson's ratio vs. Atomic Number [http:// www.webelements.com]

Bulk modulus $(\mathrm{B}$ or $\mathrm{K})$, the resistance to dilatation or volumetric change, is the volumetric resistance to pressure changes [See Figure 2-13]. Figure 2-13 shows how bulk modulus is related to the elements in the periodic chart. A high bulk modulus allows materials to resist hydrostatic compression and shear [33, 6, 6,25$]$ 


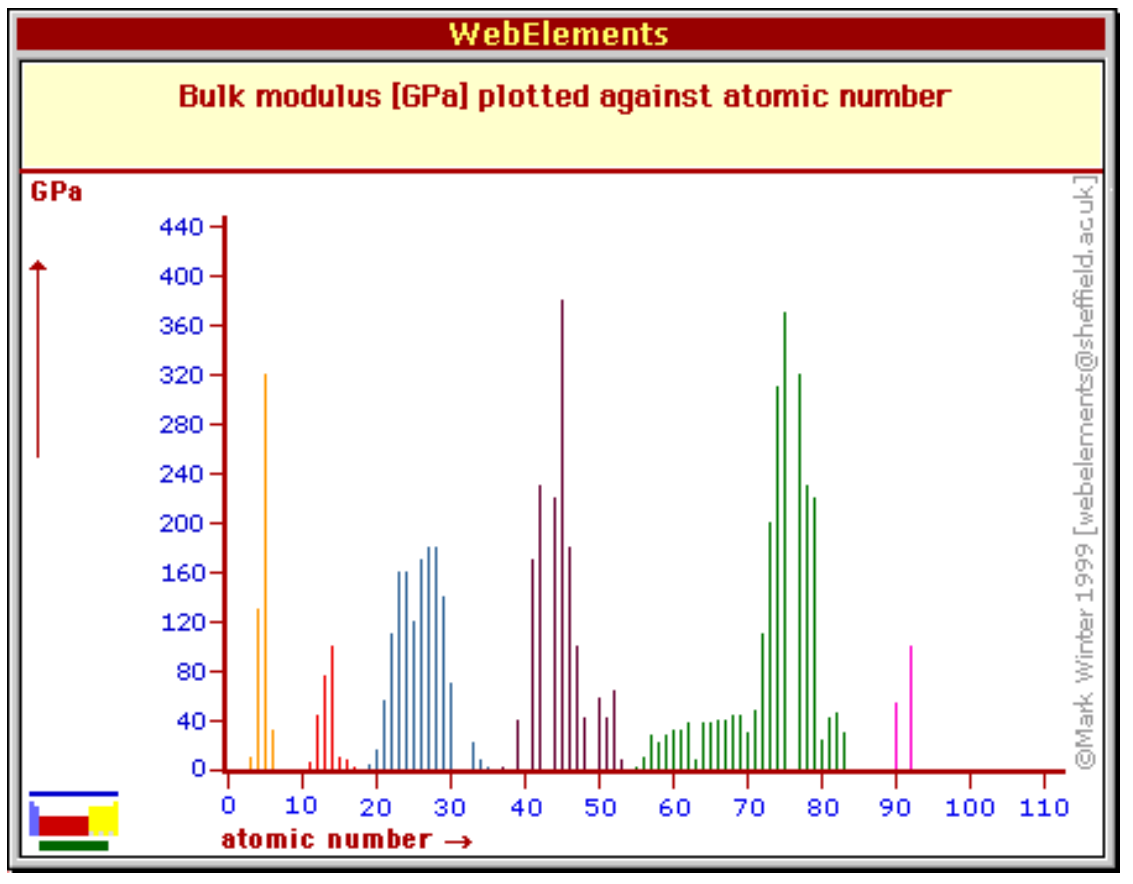

Figure 2-13. Bulk modulus vs. Atomic Number http:/ / www.webelements.com]

Shear modulus or Modulus of Rigidity $(G)$, the resistance to shape changes, is the ratio between shear stress and shear strain [See Figure 2-14] Shear modulus or modulus of rigidity is resistance to shear and is very important to plastic deformation (similar to viscosity in fluids). Figure 2-14 shows how rigidity is related to the elements in the periodic chart. A strong solid combines both a high rigidity and a high Young's modulus 53, 66, 125 . 


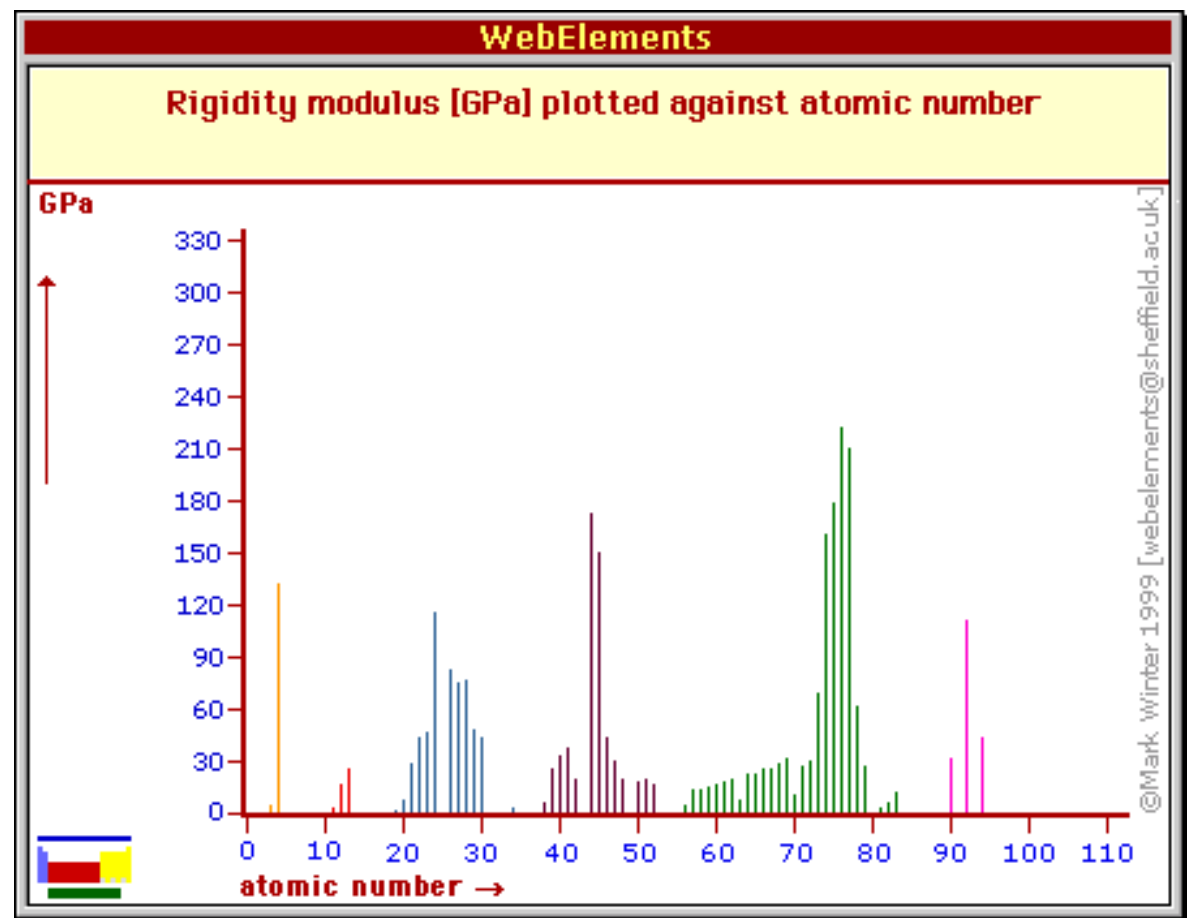

Figure 2-14. Rigidity Modulus vs. Atomic Number hhttp:/ / www.webelements.com

Hardness is the resistance to plastic deformation [See Figure 2-15 and Figure 2-16]. It cannot be understood solely in terms of classical mechanics [53]. The relative hardness of various elements is most easily visualized using Moh scale, as in Figure 2-15. The scale is approximately logarithmic, as noted in the caption, and can be compared to other hardness scales in Figure 2-16. 


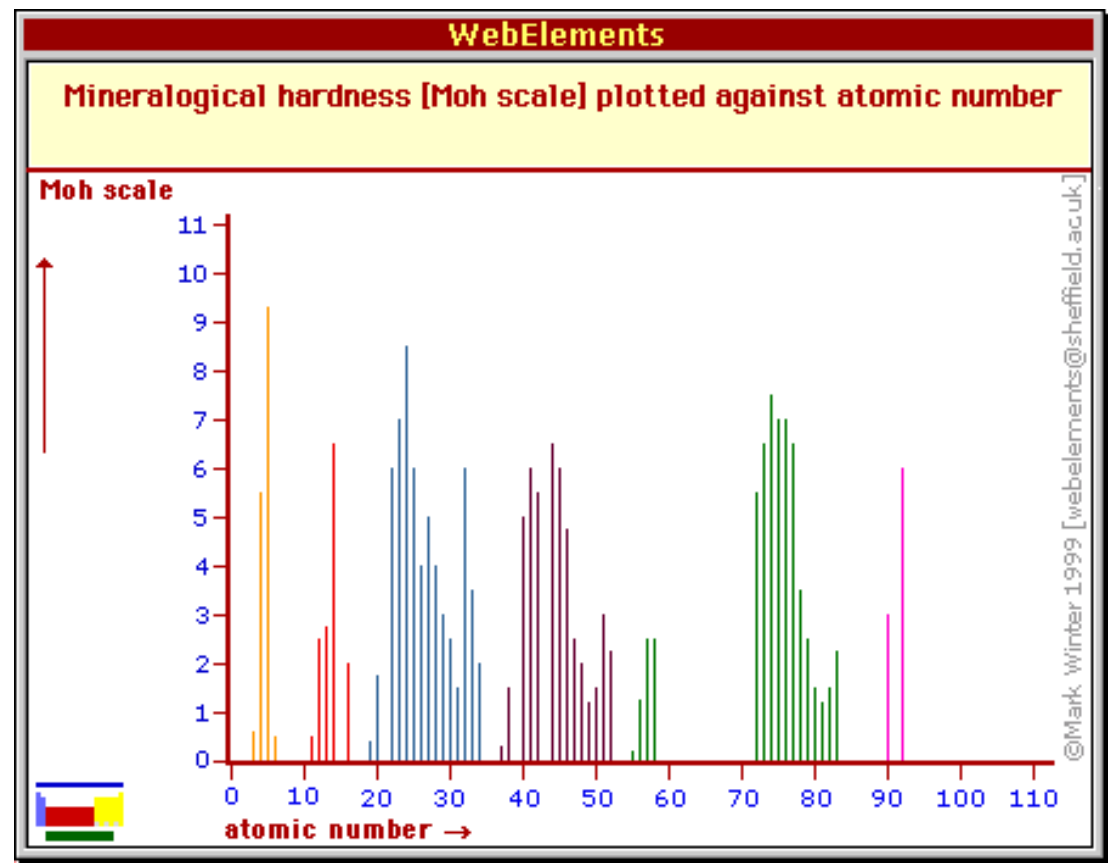

Figure 2-15. Hardness in Moh Scale vs. Atomic Number, approximately logarithmic http:/ / www.webelements.com]

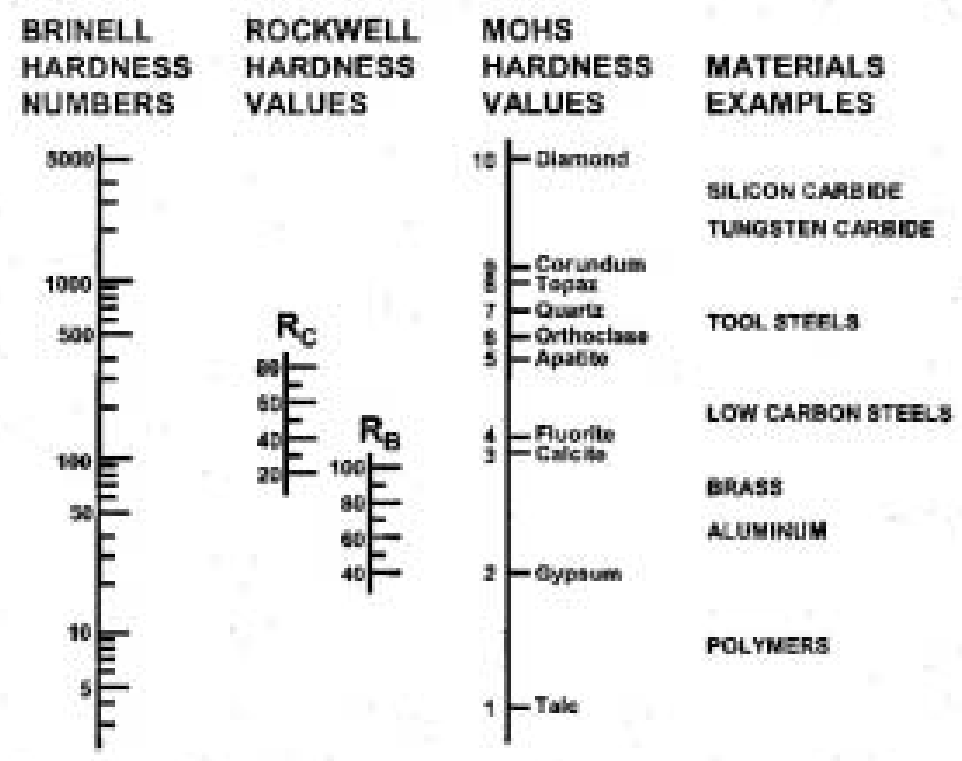

Figure 2-16. Comparison of relative hardness-Brinnell, Rockwell, and Moh scales-with material samples [127] 
Fracture toughness is the area under the stress-strain curve signifying a materials ability to absorb energy to failure (or fracture) [30, $37,5,53,59,119,130]$.

Resilience or Modulus of resiliency is the potential energy of deformation and the area under the elastic portion of the stress-strain curve $[30,37,5,53,59,119,30]$,

\subsection{Theoretical Strength}

"A brief reflection shows that 'true strength', even if it ex isted and if it could be measured crrectly, would have no practical importance for applications in engineering."

$\sim 0$ rowan 1948/ 49

\subsubsection{Egon O rowan} 16, 53, 93,88

The emphasis has been added to Orowan's discussion of the true tensile strength of materials [93]. When he wrote this passage there was indeed no practical engineering applications towards which 'true strength' theories could be applied. However, recent advances in nanotechnology coupled with the ability to manipulate individual layers of atoms and the superior strength characteristics of thin wires and perfect crystals or strong solids, leads one to the conclusion that there is an absolute need for reliable theoretical strength models [112].

Physicists use First Principles Calculations to predict properties, especially those of alloys, to reduce time in production and testing of new combinations [See nanotechnology] and 19]. Density functional theory is also leading to better modeling at the atomic level [17. 
These models yield approximate values for many important physical properties including strength, based on atomic interactions [See section 7.2 Quantum Analogy and references 2 88 .

The properties of engineering materials have been carefully catalogued through experimental methods (one such database is Stability Constants (SC-) Database) [See Section 8.0. At the micro- and nano-scale, with the possibilities of prefect crystal structure, very little of the previous experimentation is valid or extrapolatable to the smaller scales 53 , 118]. Even failure theories for bulk materials are invalidated at the smaller scales $50,53,70$, 73. 106]. This has been countered by microscale simulations using stress concentrations and embedded cracks [See Section 8.1 and references $5,42,48,50,0,13,106,112,118]$,

\subsubsection{Nucleation vs. Propagation}

Using the chemical bonds to determine strength of a material produces an ideal strength estimate, which is rarely achieved by bulk materials $[66,65,88$. Steel performs at less than 1\% of its ideal strength 93. During the 1930's a German school arguing nucleation vs. propagation of defects, attempted to explain why plastic flow occurred at a level so low in comparison with ideal strength. The theoretical strength of strong solids ${ }^{\mathrm{B}}$ was used to prove the existence of defects and failure mechanisms acting well below the theoretical limit [93]. With the advent of diffraction techniques and crystallography, this theory was validated and fracture mechanics was born [93. Fracture mechanics deals with holes, cracks, and discontinuities embedded in an ideal mechanical continuum [132]. This theory is

\footnotetext{
${ }^{3}$ Strong Solids are solid materials that are strong in tension.
} 
important to explain the low strength relative to theory and, according to Gilman 53,140 , even a perfect specimen will spontaneously produce defects when subjected to stress. Meaning even a perfect specimen cannot achieve theoretical strength.

\subsubsection{A. Kelly's "Strong Solids" [66]}

The assumption, made by Kelly $\mathrm{H}^{\mathrm{H}}$, is that any solid has a theoretical failure stress determined solely by chemical binding forces and temperature as applied to perfect specimens with no cracks, inclusions, foreign atoms, dislocations or other definable imperfections [66]. This failure stress is known as ideal strength but it does not take into account plasticity.

Ideal strength is the ultimate strength that a material can physically achieve [66]. At bulk levels, yield occurs at less than $1 \%$ for many engineering materials making this a useless calculation 93]. However, at smaller and smaller scales and under strict laboratory conditions working strength is boosted [112]. Many of the factors that make the yield strength so low at bulk scales are eliminated at small scales; for example, the probability that a defect or crack is present decreases with decreasing characteristic lengths. Also, the presence of a critical size crack is removed from samples of a size smaller than the corresponding critical size.

Some whiskers have even done the impossible and surpassed the theoretical limit [66. An order of magnitude estimate of theoretical strength was sufficient for Orowan's applications [93], but a second investigation is warranted for nano-scale engineering applications. The

\footnotetext{
${ }^{4}$ Kelly was introduced to the subject of strong solids by Cottrell who worked with Orowan [93].
} 
study of ideal strength is not to supplant current methods but to design competently at the smaller scales by knowing the upper limit of a material's strength [See Section 8.1.3 First Principles Calculations and Statistical Mechanics]. Empirical scaling laws are being formulated to scale strength from theoretical to bulk following simple scaling laws and using multiscale experimentally obtained data [0]

\subsection{Temperature Dependence}

38, 53, 87,96

Temperature is intimately related to strength. Without a temperature specification, a strength measurement is worthless, because strength is a function of temperature above the Debye temperature ${ }^{\mathrm{g}}$ 88. Below the Debye temperature, strength is independent of temperature [38]. In most common mechanics equations, temperature is not explicitly mentioned; unless the problem is one of temperature distribution, heat transfer, or thermoelastic behavior [30, 37, 25, 53, 59, 119, 130]. The assumption is that isothermal conditions exist when temperature is not explicitly mentioned $30,37,75,53,59,119,130$. Many other assumptions will be examined as they arise in this study.

Isothermal condition is a significant simplification, requiring that no temperature changes occur and that temperature is not a variable in the problem $30,37,75,53,59,119,130$. The effects of hot and cold in real life conditions are important to keep in mind, because many extraordinary failures have been the result of neglecting temperature. Low quality

\footnotetext{
${ }^{5}$ Debye temperature is a material constant that is used to calculate heat capacity $(\mathrm{Cv})$ and relates to the velocity of sound, phonon interactions, and Young's Modulus.
} 
steel becomes brittle in icy waters, causing failures in bridges, cables, ships, and airplanes. Most notably, the Titanic failure and Liberty ships during WWII were cracked open mainly due to the brittle transition of the steel used in the design [3]

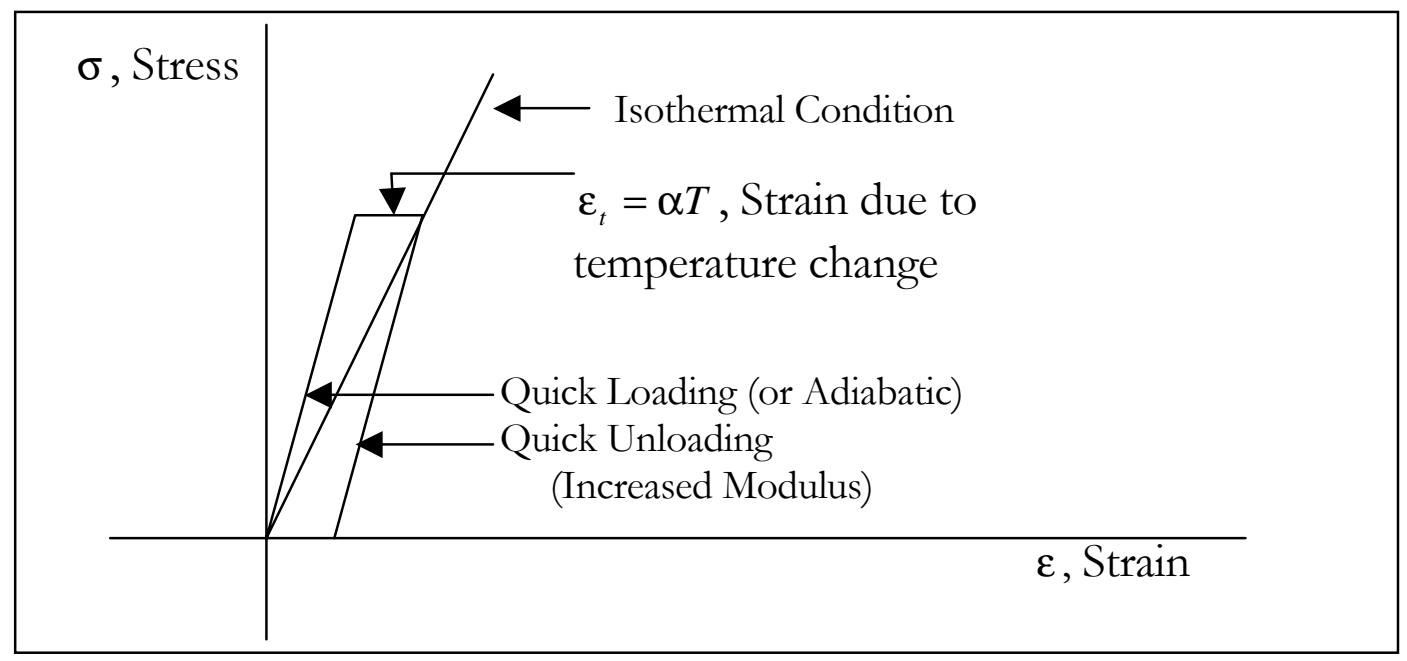

Figure 2-17. Diagram of the effect of non-isothermal conditions when a load is applied quickly [32, 75, 120, 130.

As Figure 2-17 demonstrates, the isothermal condition is not just a restriction in temperature, but in time as well. When a load is applied quickly, the ratio of stress to strain (or Young's Modulus) is increased and the temperature of the specimen drops [75]. The material is in effect stronger because it cannot respond at the rate that the load is applied [See also Eigure 2-4], If the load is maintained, when the material achieves thermal equilibrium, then an increased strain, or elastic aftereffect, is observed due to the thermoelastic behavior [75. This effect in steel is small, about $1 / 3$ of $1 \%$ elongation, but it 
can become significant in repetitive loading or vibration, because the complete cycle represents a loss of energy to internal friction which is actively damping the motion [53]. Thermoelastic material analysis is important in crack tip analysis [92], and is considered as a supplement to photoelastic experimentation [139].

The fracture or failure of a material has been likened to the dissolution of a material at a certain temperature, the melting point [38].

\subsection{Time Dependence}

60,91

Time dependence, or anelastic response, includes thermo-elastic effect, hopping of small atoms like carbon in a matrix of larger atoms, and stress induced ordering $42,53,65$. Although bonding is primarily an electrostatic phenomenon, hopping is an important electrodynamic fluctuation, listed below [53, 88].

- Electrons hopping between ions (covalent bonding)

- Photons hopping between molecules, or atoms (London or Casimir bonds)

- Protons hopping between atoms (Hydrogen bonding)

- Phonons hopping between conduction electrons (superconductivity)

In the study of elasticity, time is only explicitly stated when the load is cyclical, as in fatigue failure $30,37,75,53,59,119,130$. A before and after snapshot of a dynamic process is 
contained in the familiar state equations of stress and strain. A more exact model would be one defining the final equation of elasticity as the summation of a time dependent series that converges to a state of stress (or diverges to failure). The assumption is that enough time has passed that the final picture is one of stable equilibrium $30,37,75,53,59,119$, 130]. This assumption has proved fruitful within its field of applicability, but has complicated the theory of plasticity, where time dependence is necessary [See also 2.12 Vibration and Impact and references 5, 42, 48, 50, 70, 73, 106, 112, 118. Incubation time is proposed as a material parameter for high-rate loading leading to fracture [1]

\subsection{Plasticity} [7, 8, $16,23,6,36,39,49,0,53,60$

Plasticity is not homogeneous; it is highly irregular in creation and propagation of defects [5, $42,48,50,53,70,73,106,112,118$. Elastic deformation is usually temporary, well ordered, and propagates quickly on elastic waves, in contrast, plastic deformation is usually permanent, chaotic, and propagates relatively slowly [53]. Plasticity is the result of dissipative or non-conservative forces [See conservative force field on page 52]. The system's energy is lost to internal friction [Peierls] and bonds are broken. As a result, the material is plastically deformed; it cannot return to its original configuration without additional energy [88]. A close correlation exists between band gap and glide activation energy, the energy needed to start a dislocation moving [53, 88]. In high-rate loading, incubation time to fracture [91] is a definable material property needed to initiate failure. 
If time dependence were included in elastic equations, then the role of dissipative forces could be modeled, even when the material is not strained above the elastic limit. Time dependence is modeled when using nonlinear analysis or micro-scale modeling ,5, 42, 48 , 50, 70, 73, 24, 106, 112, 118, 128. This effect is observable in real materials, but not included in most models not dealing specifically with plasticity.

Failure is a plastic phenomenon, and the unification of failure and nonlinear elasticity produced the theory of continuum damage mechanics with special applications to rock mechanics [132]. Modeling plasticity at the microscale is very difficult in part due to highly chaotic grain boundaries [See also Figure 8-1 in Section 8.1.1 Crystal Structure], and incomplete understanding of stress transmission through grains [See also Figure 2-18] in Section 2.11 Grain Size and Figure 7-1 in Section 7.1 Fluid and Wave Analogy

\subsection{Ductile vs. Brittle}

Resistance to plastic flow cannot be explained with continuum mechanics; some materials are hard and brittle while others are soft and ductile, and the difference requires a quantum approach [53, 88]. Polarizability is inversely related to shear moduli, meaning that highly ductile materials have low polarization and brittle materials are highly polarizable [53]. Dislocation mobility also defines whether a material will be brittle or ductile $11,0,16,23,53$, 88. 26. 122. When dislocations move quickly, the material is ductile, but if barriers are present, then the material fails in a brittle manner. Where definite yield stresses are observed, there are barriers to the mobility within the specimen, defects that prevent dislocation flow $[53,88,83]$. 
"It remains to be explained why there is a size effect in notch brittleness on a centimeter scale. A square rod of $0.5 \mathrm{~m}$ with a machined notch may bend in an entirely ductile manner while a geometrically similar sample of $10 \mathrm{~cm}$ breaks ex plosively in bending tests." Timoshenk 0

The appearance of size dependent ductility suggests that when the specimen is of a scale geometrically similar to Griffith critical crack size, that there will be a corresponding notch brittleness effect $33,30,37,75,53,59,119,130$. An enhanced Griffith crack of $\sim 1 \mathrm{~mm}$ will not usually be present in a virgin specimen but must grow in a ductile manner 33 . From this it can be inferred that the high strength of wires with thickness less than or equal to $1 \mathrm{~mm}$, is due to an absence of the critical Griffith crack [3].

Orowan based his notch brittleness calculation on Hencky and Prandtl's indentation of ideally plastic solid by a rigid punch [3]

- If $\mathrm{Y}$ is yield stress, Stress-max cannot exceed 3Y, and B is brittle strength; then for $\mathrm{B}<\mathrm{Y}$ the material is brittle.

- For $\mathrm{Y}<\mathrm{B}<3 \mathrm{Y}$ the material is ductile in tensile tests, but notch brittle.

- For $\mathrm{B}>3 \mathrm{Y}$ the material is fully ductile (not notch brittle).

- Thus, B is hardly altered at low temperatures, but $\mathrm{Y}$ is roughly trebled. Therefore, a material notch brittle at room temperature will be brittle at low temperatures. 
Solidity index (S) is another method for determining brittleness and defining liquid-solid transition [53. $G$ is shear modulus and $B$ is the bulk modulus in the equation below, Equation 2-2. The most solid of materials is diamond with the maximum solidity index of 1.3; solidity of zero indicates a liquid [53]. At $S>0.23$ materials become brittle. For cubic materials, $\mathrm{G}$ equals $\mathrm{C}_{44}$ or $0.5^{*}\left(\mathrm{C}_{11}-\mathrm{C}_{12}\right)$, depending on the crystal structure; these values are equivalent for isotropic materials [53].

$$
\mathrm{S}=(3 / \pi)[\mathrm{G} / \mathrm{B}] \approx(3 / 4)[\mathrm{G} / \mathrm{B}]
$$

\section{Equation 2-2 Solidity Index [53]}

\subsection{Hardening Mechanisms-History Effects}

26. 48,122

Many time dependent processes can increase strength while molding the shape of the final part. In general, any process that increases density (and increases dislocation density) also increases the tensile modulus [53]. Working a material can work-harden or work-soften depending on the chemical composition and following the Orowan mechanism [48, 53]. By combining cold temperatures (liquid nitrogen) and cold-working, researches at JohnsHopkins were able to increase the hardness of pure copper by six times without sacrificing ductility or adding impurities, alloying 122]. Dislocations and nano-engineering were the main strengthening mechanisms. The cold slowed dislocation mobility while the cold rolling to $1 \mathrm{~mm}$ thickness created a high density of dislocations, similar to the process of folding steel [33, 122]. The thickness is significant as the apparent crossover point to increased 
strength due to absence of enhanced Griffith crack [33, 122]. The sample was then allowed to recrystallize at $200 \mathrm{C}$; this produced ultrafine grains because dislocation density before recrystallization is inversely proportional to grain size [122]. The team controlled the grain size growth to produce a bimodal mix of ultrafine and larger grains, which gave the copper its high strength (due to ultrafine grain) while retaining ductility (due to larger grain) [122]. Chaotic mixing of grain size can also create increased strength as defined in the Chaos section as related to strength on page 46.

Pure elements can be combined to form alloys with properties more desirable than their constituent elements, like steel made from iron $(\mathrm{Fe})$ and less than $6 \%$ carbon $(\mathrm{C})$ [19, 48 . High strength steels are produced by adding impurities to the compound like nickel, chromium, molybdenum, and others. Alloying increases hardness, in general, especially between atoms not located near one another on the periodic table 53,88 . This increase in critical shear stress is a result of slowing dislocation mobility by nonhomogeneous material made of dissimilar atoms [53]

\subsection{Hydrostatic Pressure}

The lesson learned from hydrostatic pressure should convince one of the importance of the gradient as compared to yield strength [See Potential Theory and Divergence]. A material subjected to intense hydrostatic pressure refuses to fail at the values obtained from failure theories, though at high enough pressures materials can compress to crystal structures that have greater stability [33, 88. Between 10 - 100 Mbar of pressure, all elements become 
metals (Herzfeld's criterion); physicists define a metal by its ability to conduct electricity [53].

\subsection{Grain Size}

Grains are single crystals made of homogenous material that are orientated [88], The size can range from microns to visible grains to single crystal parts like turbine blades. In general, the smaller the grain size, the higher the strength, with the exception that a single crystal is the strongest and most ductile form of a material [53]. Grain size is an important strength factor at any scale. A material can be strengthened by a recrystallization process that forms smaller or more symmetric grains [88, 122]. Smaller grains relieve stress concentrations from dislocation pile-ups [3].

The Hall-Petch effect states that stress $(\sigma)$ is equal to the applied stress $\left(\sigma_{0}\right)$ plus a factor that takes into account the diameter of the grain, $d$, and $K_{y}$, a constant representing the significance of grain boundaries to increased strength [p8]

$$
\begin{aligned}
& \sigma=\sigma_{0}+\frac{K_{y}}{\sqrt{d}} \text { Hall - Petch Effect } \\
& \mathrm{Y} \propto \frac{1}{\sqrt{d}} \quad \text { Hall - Petch Relation }
\end{aligned}
$$

\section{Equation 2-3 Hall-Petch Relation}

In the Hall-Petch relation $30,48,59,75$, yield stress $(\mathrm{Y})$ is proportional to the inverse of the square root of the diameter of the grain size $(d)$. The important crossover point where 
smaller diameters stop being inversely related to strength is being investigated and modeled by CMSN, the Computational Materials Science Network [73]

Existing cracks will propagate if it lowers the total energy of the system [53]. Griffith in 1920, assumed a simple energy balance, a decrease in strain energy is used to create new crack surfaces $[30,37,75,53,59,119,130]$. His theory estimates theoretical strength of brittle solids and gives the correct relationship between fracture strength and defect size [53]. This was improved upon by Irwin in 1950 by developing the stress intensity approach 30, 37, $75,53,59,119,130$.

The boundary or interface between grains is a stress concentrator [See also Eigure 8-1] in Section 8.1 .1 Crystal Structure. It is more difficult to propagate a crack through a single crystal than to separate two grains. (However, dislocations have no trouble propagating through single crystals in Figure 8-1 The boundary is less symmetric and has fewer and weaker bonds holding the grains [53, 88]. The bonds are fewer because the ideal arrangement is that of a single crystal and weaker because there is internal stress due to misalignment of cell structure [63, 65, 66, 88,. Many of the defects found in materials are concentrated at the boundaries including: interstitials, vacancies, impurities, micro-cracks, line defects, slip planes, twinning, and environmental contaminants (on the surface boundary) $[5,7,28,42,63,65,106$.

Grain size is a vital consideration in thermoelastic and heat transfer models [139]. Like elastic properties, thermoelasticity is dependent upon orientation, meaning different grains 
will possess different temperatures when the material is quickly stressed or at stress concentrations like crack tips [92]. The heat contained in a grain is per volume, while the heat exchange, is per surface area. Less surface area in smaller grains allows better heat transfer and greater loss of mechanical energy to internal friction [53]. This suggests that better damping characteristics are attainable with smaller grain size.

The experimental work done on cohesionless materials [108] suggests that different mechanics control the transmission of stress from grain to grain. Figure 2-18 shows a photoelastic stress distribution though grains. In contrast to continuum mechanical (classical elasto-plastic) models that use hyperbolic or elliptic equations, the results pictured in Figure 2-19 using photoelastic techniques, show a diffusive transmission of stress through the medium entirely contained within a parabola [108]. Continuum mechanics predicts a bell shaped or gaussian distribution for the same boundary conditions while the relatively recent hyperbolic model would propagate along rays [108]. This result is not apparent in the individual tests, but in the superimposed, ensemble averaged results from different grain configurations [108]. These results have been used to model granular materials with applications in soil mechanics, but the findings bear relationships to solid materials composed of grains. These grains of solids resist sliding and shear deformation better than the test materials, but the final response may be a linear combination of diffusive stress transmission and the resistance to grain slide or shear.

This diffusive model can be simulated using cellular automaton methods and the diffusion equation is part of potential theory [See Section 7.0 Analogous Systems]. The ensemble 
averaging technique [108] is similar mathematically to probability density used in statistical mechanics $83,88,103$. The shifting of the grains is like running a probability experiment to produce general behavior, since the exact path of stress transmission through the material is random but follows a fundamental probability distribution over many experiments. Even though real materials have different sized grains, defects, and nonhomogeneous distributions in the layers, this diffusive model suggests an interesting relationship that should be examined further. Photoelastic experiments on indentation tests at the micro-level could demonstrate whether this phenomenon is transferred to solids through the response of grains, and ensemble averaging appears to be an excellent technique to produce characterization and probability of response.

Steel is the favored material for mechanical engineers; much of the curriculum, literature, and experimental work are devoted to steel and it's alloys. However, the properties, which make steel ideal for an engineering material, are grain dependent and therefore a micro-scale phenomenon. At the nano-scale, steel will not be as important as silicon or more stable, covalently bonded materials. 


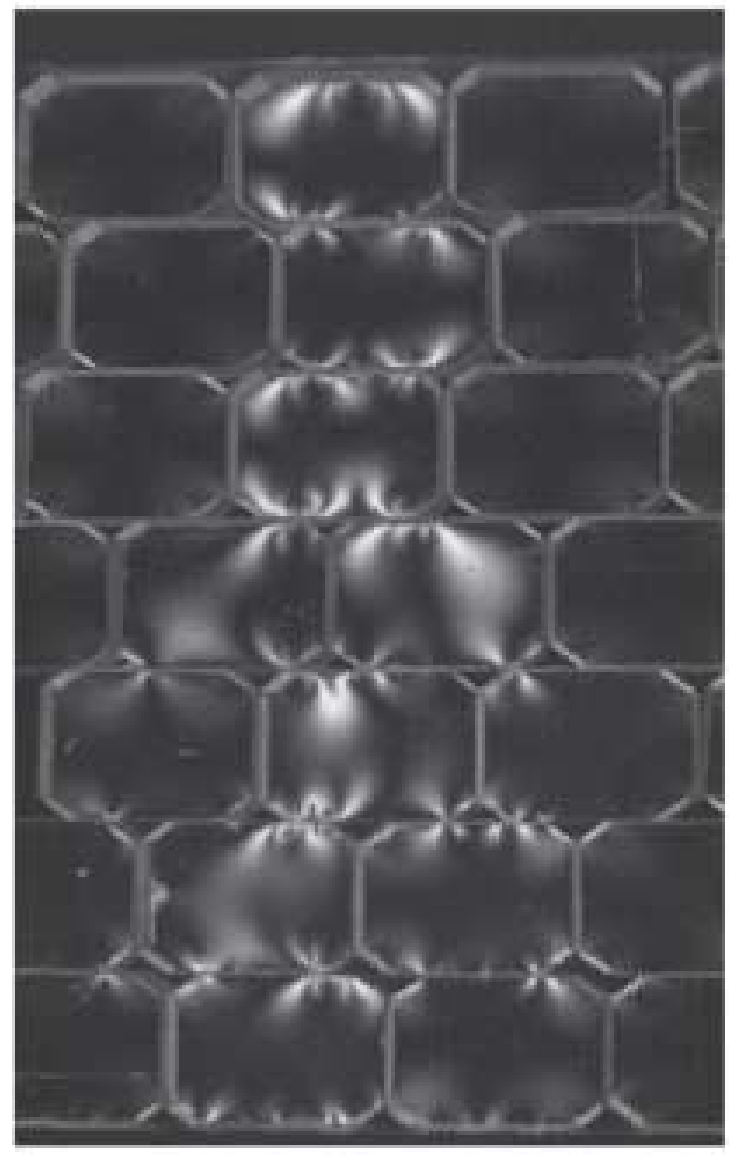

Typical photoelastic view of a packing submitted to a point load of $20 \mathrm{~N}$. Each packing is made up of regular arrays of square grains; there is an arbitrary shitt between adjacent arrays. Owing to tip ffects, the real contacts that grains experience are preferentielly located at their vertices.

Figure 2-18. Typical Photoelastic results of the stress transmission experiment through a cohesionless material [108]

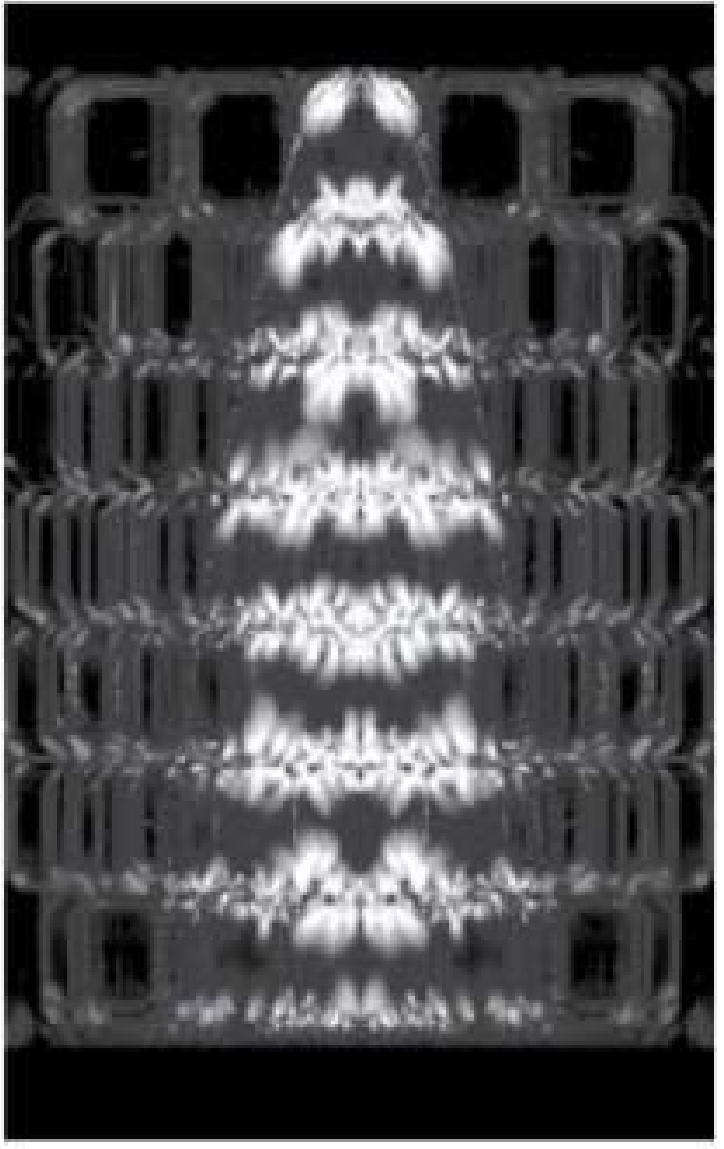

Superimposition of ten digitized pictures. Each packing corresponds to a different arbitrary. shift between horizontal adjacent layers. Th strained region appears to be bounded by a parabola that we represent by a dashed line, and which is a guide for the eyes. The bounding parabola obeys the equation $x=\sqrt{2 D y}$ (with $D$ $=0.45 \pm 0.05$ grain size). 


\subsection{Vibration and Impact}

$8,11,13,20,27,36,45,129,134$

A common mechanical engineering experiment to determine tensile modulus involves measuring the speed with which a vibration/impact travels through a known length of material. This acquired modulus is actually higher than that obtained from isothermal tensile tests, and the difference can be used to determine the ratio of specific heat at constant stress (pressure) to the specific heat at constant volume [75. At the nanoscale, it is much more difficult to calculate the amplitude of vibration [11], if this were possible then the exact strength of atomic bonds could be experimentally obtained by using a microbalance, QCM, instead of the relative bond strength [See Section 8.2 Material Advances in Nanotechnology, Section 1.3 Examples of Specific Nanotech Problems, and Reference 11].

Time dependence becomes very clear during impact experiments [106]. The speed of the applied load is the determining factor to the response of the system. At relatively slow speeds, thermoelastic response is minimal, but at high rates, material properties become rate dependent, already demonstrated in Eigure 2-4 [B3]. During impact, a material's strength is increased and ductile materials can respond explosively brittle. The strength increase is mostly due to the inability to respond to the force as quickly as it is applied. This resembles the resistance to failure of materials subjected to large hydrostatic forces [10],

\subsection{Chaos}

Another aspect that requires attention is Chaos and Nonlinearity. Chaos has built in scaling phenomena, and where there is nonlinearity, there is chaos. Bifurcation is the route 
to chaos 94, when two equilibrium states are separated by an infinitesimal amount. However, chaos does not mean instability, a chaotic system can recover from small perturbations demonstrating global stability even with local unpredictability. Examples of this are found throughout nature: the weather, the movements of the planets, the movement of a pendulum (large angle), the human heart, oscillating circuits, frequency locking, and dripping faucets [54]. Therefore, to retain this rich behavior in mechanical systems, the nonlinear terms should be retained in the stress equations [Equation 3-12, 94 . Nonlinear terms drop out in situations where the simplifying assumptions are valid, making the cut-off points for the applicability of linear equations more obvious [p4],

In the case of an aluminum-based compound [09], neutron scattering aided the production of an amorphous rather than crystalline structure, which proves to be more elastic (higher Young's modulus) than the same material with grain boundaries present [See Section 2.2 .2 Crystallography-Geometry]. Neutron scattering, one of the diffraction techniques for visualizing micro-scales and molecular dynamics, is a very important testing technique for studying property or phase changes and for producing disordered (chaotic) or amorphous materials with higher strength and resistive properties [69, 122]. Some applications include: improving cobalt- and titanium-based medical engineering materials, testing of degradation of materials responsible for containment of radioactive materials, and amorphous semiconductors [69, 102, 104].

Glass is the most common example of an amorphous material and has been described as super-cooled or frozen liquid, but this comparison (or the fluid analogy) should not confuse 
the liquid and solid states of matter [56]. Glass may have the amorphous form of a liquid but it has a much higher viscosity [6, 88 ,

$\begin{array}{ll}\text { At room temperature: } & \text { Viscosity: } \\ \text { Water } & 0.01 \text { poise } \\ \text { Lead } & \sim 10^{10} \text { poise } \\ \text { Glass } & \sim 10^{20} \text { poise } \\ \text { Glass@ melting temperature. } & \sim 5^{*} 10^{7} \text { poise }\end{array}$

Note: at room temperature it would take longer than the lifetime of the universe for glass to flow a noticeable distance and the waves present in glass is due entirely to the manufacturing process [0]

Adobe is an example of how chaotic mixing can increase the strength of a material 54, 102. The constituents of adobe are soft, but the mixture is like concrete [54, 102]. Large grains are held together by small grains, which are in turn surrounded by smaller grains. This scaling pattern has an optimum mixture to obtain the best building qualities of the mixture [54, 79,80 102]. Metals can benefit from this study as demonstrated in [122] where pure copper's hardness was increased without sacrificing ductility by controlling grain growth and mixture. 
Fracture progresses in an entirely chaotic fashion, apparently random, but cellular automaton efforts have been made to model the random walk behavior of crack propagation [102,138].

\subsection{Failure}

Failure criteria were developed closely following experimental evidence and work well (if selectively) applied to bulk materials [53. Steps have been made in computational mechanics to bridge the steps from nano- to micro- to macro/bulk behavior [5, 42, 48, 50, $70,73,106,112,118$. To date, the transitions have not been accomplished smoothly. At the limit, nano-mechanics must describe bulk behavior or continuum mechanics; just as quantum mechanics is expected to describe macroscopic behavior or Newtonian physics in its limit. For this reason, potential theory is ideal for modeling material response. The same equations and numerical methods that are used to solve quantum phenomena are applicable to bulk behavior [See Section 4.0 Potential Field Theory Applied to Mechanics]

Testing has begun on tiny cantilever beams, thin films, and quantum structures in an effort to understand micro- and nano-mechanics [23. 113. This testing is currently being conducted to produce a data set that can be fit by a new failure theory, proceeding in the same manner that has worked for bulk materials, albeit with smaller, more sensitive, and more expensive testing apparatus. Following this lead, each new nano-component will have to be run through a battery of tests comparable to the past 200 years of bulk material testing and performed at enough different scales that a sensible understanding of properties can be assembled before design can be attempted. While experimental testing is an 
important part of design, computer modeling $[5,42,48,50,70,73,106,112,118$, could take over most of the tedious job of producing property curves. This of course necessitates that the modeling is accurate and produces a good representation of nano- and micro-scale effects.

At the atomic level, failure is not isotropic [See also Figure 8-1] in Section 8.1.1]. There are not enough grains for a homogenous mix, and failure stress is highly dependent on orientation of crystals [See Section 3.2.5 Constants]. Quantum mechanical effects also appear in a tangible way $12,17,20,23,32,38,39,42,43,44,49,50,51,53,63,66,69,73$, 83. 26, 102, 103, 104, 106, 107, 112, 118, 123, 133, 140.

Most failure theories do not account for differences in tensile or compressive loading, allowing the tensile curve to reflect across the strain axis. However, many materials are stronger in compression than tension, and even stronger than theory predicts for hydrostatic compression. These factors need to be addressed in modeling compression and hysteresis effects [49]

\subsection{Conclusions about Strength}

Although the idea of a material performing at a percentage of its maximum, or ideal strength, is not important for bulk calculations, it has become important to nano-scale calculations and especially in modeling material response. Each of the variables that contribute to strength must be included in a comprehensive model, not only to demonstrate their effect, but also to observe the limiting conditions (where the variables 
can safely be eliminated). Some excellent modeling programs have been developed exclusively for the atomic and molecular scale for chemists and physicists [See also Figure 8 -2 on page 127 and Section 8.1 Applied Math and Modeling. These can serve as the first level to modeling independent of scale and an aid to engineers working and designing with nano-components.

\subsection{Further References}

See references to the works sited in this chapter, since many are collections of years of historical work, especially Love [5] and Timoshenko [130] 


\subsection{Literature review and Denivation of Elasticity}

The founding fathers of this science were indistinguishable from physicists, and most of their principles were formulated without a dependence on scale, but some of the assumptions preclude the use of these equations at smaller scales $[2,55,57,58,75,64,93$. 130]. In each of the following sections, these limitations are highlighted.

\subsection{Consenvative Field}

If in a gradient force field $\mathrm{F}$, the work done by the force upon a particle moving from $\mathrm{A}$ to $\mathrm{B}$ is the same for all paths, and work done on a closed path is zero, then the force field is said to be conservative 75,143 . Work is done when an object or particle is moved by a force in the direction of that force and the units are energy [119, 143]. Power is work by time or distance times velocity $[30,37,75,33,59,119,130,43]$

$\mathrm{F}$ is conservative if and only if curl $\mathrm{F}=0$ [See url on page 9 ?nd reference 143]

Conservation of energy requires that the sum of all energy, kinetic (KE) and potential (PE), be constant in any closed system.

$$
\mathrm{KE}+\mathrm{PE}=\text { constant }
$$

Equation 3-1 Conservation of Energy

Frictional forces are non-conservative or dissipative; meaning $\mathrm{KE}$ is reduced without an increase in PE and work is path dependent [43]. 
$\oint_{c} F \cdot d r \neq 0 \quad$ for a non-conservative field

Equation 3-2 N on-conservative field

$\oint_{c} F \cdot d r=0 \quad$ for a conservative field and $\mathrm{F}$ is the gradient of a scalar function

and circulation is zero [143].

Equation 3-3 Conservative field

In a three-dimensional conservative field where $\mathrm{F}$ is a gradient field; $\Phi$ is a potential function for F; and P, Q, R are components.

If $\mathrm{F}=\mathrm{Pi}+\mathrm{Qj}+\mathrm{Rk}$ and $\mathrm{P}, \mathrm{Q}, \mathrm{R}$ are $f(\mathrm{x}, \mathrm{y}, \mathrm{z})$, then:

$$
\frac{\partial P}{\partial y}=\frac{\partial Q}{\partial x}, \frac{\partial Q}{\partial z}=\frac{\partial R}{\partial y}, \frac{\partial R}{\partial x}=\frac{\partial P}{\partial z}
$$

Equation 3-4 Conservative field denivatives

Conservative Field Assumption is invalidated at microscale where plastic deformations can occur at any temperature, sometimes with no load [53. Figure 8-1 in Section 8.1 .1 is from a simulation, but is visually representative of this phenomenon.) Defects, cracks, and dislocations are topological disorders that result in the material not simply connected, thus path dependent. Plasticity is the evolution of a material not in equilibrium.

\subsection{Equilibrium}

30, 37, 59,85 
Stress Equations of Equilibrium, Equation 3-5, can be derived by isolating an element in the body; the summation of forces in each direction must be zero to maintain equilibrium 30, 37, $55,53,59,119,130$;:

\subsubsection{Stress fields:}

$$
\begin{aligned}
& \frac{\partial \sigma_{x x}}{\partial x}+\frac{\partial \tau_{y x}}{\partial y}+\frac{\partial \tau_{z x}}{\partial z}+F_{x}=0 \\
& \frac{\partial \tau_{y x}}{\partial x}+\frac{\partial \sigma_{y y}}{\partial y}+\frac{\partial \tau_{y z}}{\partial z}+F_{y}=0 \\
& \frac{\partial \tau_{z x}}{\partial x}+\frac{\partial \tau_{y z}}{\partial y}+\frac{\partial \sigma_{z z}}{\partial z}+F_{z}=0
\end{aligned}
$$

\section{Equation 3-5 Equilibrium stress fields}

Equilibrium Assumption, Equation 3-5, requires that the body is at a constant state of equilibrium, but at nanoscales, the isolated element is in a different state of equilibrium. Stress fluctuations are caused by vibrating atomic components 51, 53, 60, 88. This is a natural state of matter, and even if the material is theoretically cooled to absolute zero, there are still quantum vibrations [83, 88].

Summation of moments leads to the conclusion that:

$$
\begin{aligned}
& \tau_{y x}=\tau_{x y} \\
& \tau_{y z}=\tau_{z y} \\
& \tau_{z x}=\tau_{x z}
\end{aligned}
$$

\section{Equation 3-6 Summation of moments}


This, Equation 3-6, prevents the body from rotating due to unbalanced shearing forces 30 , 37, $75,53,59,119,130]$.

\subsubsection{Body Force Fields}

$$
\begin{gathered}
\Omega=f(x, y, z) \\
F_{x}=-\frac{\partial \Omega}{\partial x}, \quad F_{y}=-\frac{\partial \Omega}{\partial y}, \quad F_{z}=-\frac{\partial \Omega}{\partial z}
\end{gathered}
$$

\section{Equation 3-7 Body force fields}

The body forces, $F_{x}, F_{y}, F_{z}$, are a function of the body force field, $\Omega$, Equation 3-5. The units of $\Omega$ are pressure or force per unit area, and the units of body forces are in force per unit volume. They are a result of mass in a field that can be gravitational, centrifugal, magnetic, or any inertial force that adds acceleration to the element. They differ from surface forces in that they are applied by a field rather than at the surface of the body. Surface forces translate into stress fields contained in Equation 3-5. When weight is neglected, body forces are set to zero. $[30,37,[5,[3,[9,119,130]$

Body Force Fields Assumption must be adjusted to accommodate different fields at smaller scales. Gravity is insignificant at nanoscales; no atomic structure can be built thinking in terms of gravity because electromagnetic forces are so much stronger at that scale, by a factor of approximately $10^{39}$ for electrons and protons [119]. Important frictional forces include: photons (quanta of light), phonons (temperature or quanta of lattice oscillations), and plasmons (quanta of plasma oscillations of electron gas) [8, 19] 


\subsubsection{Strain - Displacement}

The derivations of elasticity theory can be found in any elementary text on elasticity, but the main points have been highlighted with their nonlinear counterparts to observe simplifying assumptions and similarities to electromagnetic fields [30, 37, 75, 33, 29, 119, 130]. The source of the Strain-Displacement relations can be followed very clearly in [94] and [75]. In the following equations, $(\mathrm{x}, \mathrm{y}, \mathrm{z})$ are the coordinate axis and $\mathrm{u}, \mathrm{v}, \mathrm{w}$ are the displacements. In the nonlinear formulations, the displacements $(\mathrm{u}, \mathrm{v}, \mathrm{w})$ can be functions of $\mathrm{x}, \mathrm{y}, \mathrm{z}$. The linear strains are denoted by $\mathrm{e}$ while the nonlinear strains are $\varepsilon$ (Greek e) to avoid confusion. Shear strains are denoted by $\gamma$, and rotations by $\omega$.

$$
\begin{array}{rlrl}
e_{x x}=\frac{\partial u}{\partial x} & \varepsilon_{x x} & =\frac{\partial u}{\partial x}+\frac{1}{2}\left[\left(\frac{\partial u}{\partial x}\right)^{2}+\left(\frac{\partial v}{\partial x}\right)^{2}+\left(\frac{\partial w}{\partial x}\right)^{2}\right] \\
e_{y y}=\frac{\partial v}{\partial y} & \varepsilon_{y y} & =\frac{\partial v}{\partial y}+\frac{1}{2}\left[\left(\frac{\partial u}{\partial y}\right)^{2}+\left(\frac{\partial v}{\partial y}\right)^{2}+\left(\frac{\partial w}{\partial y}\right)^{2}\right] \\
e_{z z}=\frac{\partial w}{\partial z} & \varepsilon_{z z}=\frac{\partial w}{\partial z}+\frac{1}{2}\left[\left(\frac{\partial u}{\partial z}\right)^{2}+\left(\frac{\partial v}{\partial z}\right)^{2}+\left(\frac{\partial w}{\partial z}\right)^{2}\right] \\
- & \varepsilon_{x y}=\left(\frac{\partial u}{\partial y}+\frac{\partial v}{\partial x}\right)+\frac{\partial u}{\partial x} \frac{\partial u}{\partial y}+\frac{\partial v}{\partial x} \frac{\partial v}{\partial y}+\frac{\partial w}{\partial x} \frac{\partial w}{\partial y} \\
e_{x y}=\gamma_{x y}=\frac{\partial u}{\partial y}+\frac{\partial v}{\partial x} & \varepsilon_{x z}=\left(\frac{\partial u}{\partial z}+\frac{\partial w}{\partial x}\right)+\frac{\partial u}{\partial x} \frac{\partial u}{\partial z}+\frac{\partial v}{\partial x} \frac{\partial v}{\partial z}+\frac{\partial w}{\partial x} \frac{\partial w}{\partial z} \\
e_{y z}=\gamma_{z y}=\frac{\partial v}{\partial z}+\frac{\partial w}{\partial y} & \varepsilon_{y z}=\left(\frac{\partial v}{\partial z}+\frac{\partial w}{\partial y}\right)+\frac{\partial u}{\partial y} \frac{\partial u}{\partial z}+\frac{\partial v}{\partial y} \frac{\partial v}{\partial z}+\frac{\partial w}{\partial y} \frac{\partial w}{\partial z}
\end{array}
$$

Equation 3-8 Linear straindisplacement
E quation 3-9 N on-linear straindisplacement 


$$
\begin{aligned}
& 2 \omega_{x}=\frac{\partial w}{\partial y}-\frac{\partial v}{\partial z} \quad \frac{1}{2} \gamma_{x y}+\omega_{z}=\frac{\partial v}{\partial x}, \quad \frac{1}{2} \gamma_{x y}-\omega_{z}=\frac{\partial u}{\partial y} \\
& 2 \omega_{y}=\frac{\partial u}{\partial z}-\frac{\partial w}{\partial x} \quad \frac{1}{2} \gamma_{x z}+\omega_{y}=\frac{\partial u}{\partial z}, \quad \frac{1}{2} \gamma_{x z}-\omega_{y}=\frac{\partial w}{\partial x} \\
& 2 \omega_{z}=\frac{\partial v}{\partial x}-\frac{\partial u}{\partial y} \quad \frac{1}{2} \gamma_{z y}+\omega_{x}=\frac{\partial w}{\partial y}, \quad \frac{1}{2} \gamma_{z y}-\omega_{x}=\frac{\partial v}{\partial z}
\end{aligned}
$$

Equation 3-10 Linear rotationdisplacement

$$
\begin{aligned}
& \varepsilon_{x x}=e_{x x}+\frac{1}{2}\left[\left(e_{x x}\right)^{2}+\left(\frac{1}{2} \gamma_{x y}+\omega_{z}\right)^{2}+\left(\frac{1}{2} \gamma_{x z}-\omega_{y}\right)^{2}\right] \\
& \varepsilon_{y y}=e_{y y}+\frac{1}{2}\left[\left(e_{y y}\right)^{2}+\left(\frac{1}{2} \gamma_{x y}-\omega_{z}\right)^{2}+\left(\frac{1}{2} \gamma_{z y}+\omega_{x}\right)^{2}\right] \\
& \varepsilon_{z z}=e_{z z}+\frac{1}{2}\left[\left(e_{z z}\right)^{2}+\left(\frac{1}{2} \gamma_{x z}+\omega_{y}\right)^{2}+\left(\frac{1}{2} \gamma_{z y}-\omega_{x}\right)^{2}\right] \\
& \varepsilon_{x y}=\left(\gamma_{x y}\right)+e_{x x}\left(\frac{1}{2} \gamma_{x y}-\omega_{z}\right)+\left(\frac{1}{2} \gamma_{x y}+\omega_{z}\right) e_{y y}+\left(\frac{1}{2} \gamma_{x z}-\omega_{y}\right)\left(\frac{1}{2} \gamma_{z y}+\omega_{x}\right) \\
& \varepsilon_{x z}=\left(\gamma_{x z}\right)+e_{x x}\left(\frac{1}{2} \gamma_{x z}+\omega_{y}\right)+\left(\frac{1}{2} \gamma_{x y}+\omega_{z}\right)\left(\frac{1}{2} \gamma_{z y}-\omega_{x}\right)+\left(\frac{1}{2} \gamma_{x z}-\omega_{y}\right) e_{z z} \\
& \varepsilon_{y z}=\left(\gamma_{z y}\right)+\left(\frac{1}{2} \gamma_{x y}-\omega_{z}\right)\left(\frac{1}{2} \gamma_{x z}+\omega_{y}\right)+e_{y y}\left(\frac{1}{2} \gamma_{z y}-\omega_{x}\right)+\left(\frac{1}{2} \gamma_{z y}+\omega_{x}\right) e_{z z}
\end{aligned}
$$

\section{Equation 3-12 N on-linear strain to linear strain and rotation [94]}

Roughly speaking the linear equations are used for the deformation of massive bodies whereas

the nonlinear equations are useful for flexible bodies especially with large deflections or rotations [94]. 
Strain-Displacement Assumption applies to small scales but many nano-structures, like carbon nanotubes and thin films, have nonlinear geometry, and require the more difficult nonlinear formulas.

\subsubsection{Hooke's Law}

-Stress is a linear combination of strain.

For many materials acting within elastic limits, Hooke's Law is a good approximation 30, 37, 75, 53. 59. 119, 130. Some theorize that it is the first term of a Taylor expansion, but for small displacements the elastic portion of a material's response is such a small strain, second order corrections are too small to measure. The constants of Section 3.2 .5 follow directly from Hooke's Law.

\subsubsection{Constants:}

Continuum solid mechanics cannot account for the magnitudes of the tensor coefficients, thus it is incomplete. A theory of coefficients requires quantum mechanics to show how atomic bonding energies and bonding forces arise $[2,17,20,23,32,38,39,42,43,44,49,50,51,53,63$, 66, 69, $73,83,06,02,103,104,106,107,112,118,123,133,140,$.

In indicial or tensor notation, the relationships and constants (stiffnesses or compliances) between stress and strain can be compactly written as follows:

$$
\begin{array}{ll}
\mathrm{i}, \mathrm{j}, \mathrm{k}, \mathrm{l}, \mathrm{m}, \mathrm{n} & =1,2,3 \\
\sigma_{i j}=\sum_{k l} C_{i j k l} \boldsymbol{\varepsilon}_{k l} & \text { Elastic stiffnesses }\left(\mathrm{C}_{\mathrm{ijk} k}\right)
\end{array}
$$




$$
\begin{array}{ll}
\varepsilon_{i j}=\sum_{k l} S_{i j k l} \sigma_{k l} & \text { Elastic compliances }\left(S_{\mathrm{ijk} k}\right) \\
C_{i j k l} S_{i j m n}=\delta_{k n} \delta_{l m} & \text { Inversion equation: } \begin{array}{l}
\delta_{k n}=1 \text { if } \mathrm{k}=\mathrm{m} \\
\delta_{k n}=0 \text { if } \mathrm{k} \neq \mathrm{m}
\end{array}
\end{array}
$$

Equation 3-13 Stress-stiffness and strain-compliance relationships [3]

The most general description of the elastic state requires the complete state of stress and strain, each containing nine components leading to a general matrix of 81 components. This can be reduced to a 6 by 6 matrix by symmetry, equilibrium, and no net torque $\left(\sigma_{i j}=\sigma_{j i}\right)$. Since elastic deformation is reversible, the strained body contains strain energy that is a scalar proportional to the square of the strain. This requires that the 6 by 6 matrix be symmetric leaving 21 independent constants, Equation 3-14 $30,37,[5,33,5,19,130$

$\sigma_{11}$
$\sigma_{22}$
$\sigma_{33}$
$\tau_{12}$
$\tau_{23}$
$\tau_{13}$$\left[\begin{array}{llllll}C_{1111} & C_{1122} & C_{1133} & C_{1112} & C_{1123} & C_{1113} \\ & C_{2222} & C_{2233} & C_{2212} & C_{2223} & C_{2213} \\ & & C_{3333} & C_{3312} & C_{3323} & C_{3313} \\ & & & C_{1212} & C_{1223} & C_{1213} \\ & s y m & & & C_{2323} & C_{2313} \\ & & & & & C_{1313} \\ \gamma_{32} & \gamma_{23} \\ & & & & & \end{array}\right.$

Equation 3-14 Triclinic elastic stiffness matrix [3] This matrix, Equation 3-14 describes the most general triclinic crystal symmetry system with three crystal axes of differing length and three different angles between the axes (crystal symmetry). For orthorhombic symmetry, nine coefficients are necessary; for tetragonal symmetry only six coefficients; and for cubic, three coefficients are needed, Equation 3-15] 30, $37,75,53,59,119,130$. 


$$
\begin{aligned}
& \sigma_{11} \\
& \sigma_{22} \\
& \sigma_{33} \\
& \tau_{12} \\
& \tau_{23} \\
& \tau_{13}
\end{aligned}\left[\begin{array}{cccccc}
C_{1111} & C_{1122} & C_{1122} & 0 & 0 & 0 \\
& C_{1111} & C_{1122} & 0 & 0 & 0 \\
& & C_{1111} & 0 & 0 & 0 \\
& & & C_{1212} & 0 & 0 \\
\\
& & & & C_{1212} & 0 \\
& & & & & C_{1212}
\end{array}\right] \begin{gathered}
e_{11} \\
e_{22} \\
\gamma_{12} \\
\gamma_{13}
\end{gathered}
$$

Equation 3-15 Cubic elastic stiffness matrix

For cubic systems, there are only three independent constants, but isotropic crystals can be further simplified since $C_{1111}=C_{1122}+2 C_{1212}$, reducing the constants to two $30,37,53,59$ 119. 130. This definition is important in experimentation, because the coefficients can be measured [37, and determining how isotropic a material behaves, justifies the use of simplified equations based on isotropic behavior. Using the isotropic assumption sets Poisson's ratio to 0.3, but many materials do not obey this rule as observed in Eigure 2-12 from Section 2.3.

Constants Assumption of stiffnesses and compliances is useful for crystalline microstructure, but isotropy is not very common, especially at smaller scales.
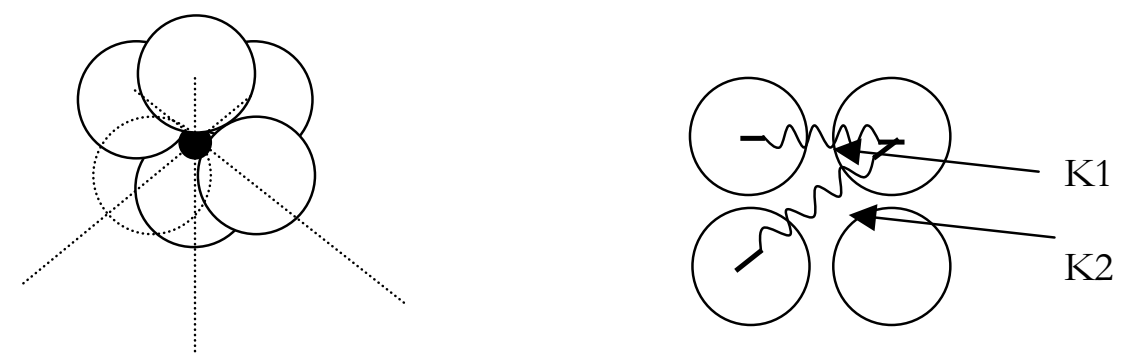

Figure 3-1. Diagram of the atomic bonds in a cubic isotropic crystal

$\mathrm{K} 1$ and $\mathrm{K} 2$ in Figure 3-1 are linear spring constants representing central atomic forces between atoms $30,37,75,53,59,119,130]$. For an isotropic crystal, K1 must equal K2 [53]. For identical 
atoms, the geometry must be skewed to be isotropic, but ionic compounds can use the geometry illustrated in Figure 3-1 on the left. The anisotropy coefficient is:

$$
\mathrm{A}=2 \mathrm{C}_{1212} /\left(\mathrm{C}_{1111}-\mathrm{C}_{1122}\right)
$$

\section{Equation 3-16 Anisotropy coefficient [53]}

When $\mathrm{A}$ is equal to 1 , then the material is completely isotropic: tungsten is 1; diamond is 1.6; and salt, $\mathrm{NaCl}$, is 0.7 [53].

The two Constants for isotropic materials can be defined by any combination of the following 37 :

- Lame's constant $(\lambda)$ with no physical meaning, used to simplify equations and is equal to $\mathrm{K}-2 / 3 \mu$

- Shear Modulus or Modulus of Rigidity ( $\mu$ or $\mathrm{G}$ ) equal to shear stress by shear strain= $\tau / \gamma$

- Poisson's Ratio $(v)$ equal to transverse strain by axial strain $=\varepsilon_{x x} / \varepsilon_{y y}$

- Young's Modulus (E) equal to Axial stress by axial strain $=\sigma_{x x} / \varepsilon_{x x}$

- Bulk Modulus (K or B) equal to Hydrostatic Pressure by volume dilatation=-p/D

Figure 3-2 shows the relationship and conversions between these constants; only two of which are independent in isotropic crystals. 


\section{Relationships between the elastic constants}

\begin{tabular}{|c|c|c|c|c|c|}
\hline & $\begin{array}{l}\lambda \\
\text { equals }\end{array}$ & $\begin{array}{l}\mu \\
\text { oquals }\end{array}$ & $\begin{array}{l}E \\
\text { equals }\end{array}$ & equals & $\begin{array}{l}K \\
\text { equals }\end{array}$ \\
\hline$\lambda \mu$ & $t^{*}$ & & $\frac{\mu(3 \lambda+2 \mu)}{2+\mu}$ & $\frac{\alpha}{2(\lambda+\mu)}$ & $\frac{3 \lambda+2 \mu}{3}$ \\
\hline$\lambda, E$ & & $\frac{A++(E-3 \lambda)}{4}$ & & $\frac{A t-(E+\lambda)}{4 i}$ & $\frac{A++(3 A+E)}{6}$ \\
\hline LV & 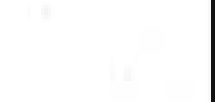 & $\frac{\lambda(1-2 v)}{2 r}$ & $\frac{\lambda(1+v)(1-2 v)}{v}$ & & $\frac{2(1+v)}{3 v}$ \\
\hline$\lambda K$ & & $\frac{3(K-\lambda)}{2}$ & $\frac{9 K(K-\lambda)}{3 K-\lambda}$ & $\frac{\lambda}{3 K-\lambda}$ & - \\
\hline$\mu E$ & $\frac{\mu(2 \mu-E)}{E-3 \mu}$ & & & $\frac{E-2 \mu}{2 \mu}$ & $\frac{\mu E}{3(3 \mu-E)}$ \\
\hline$\mu, v$ & $\frac{2 \mu v}{1-2 v}$ & 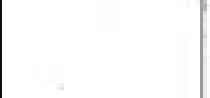 & $2 \mu(1+v)$ & & $\frac{2 \mu(1+v)}{3(1-2 v)}$ \\
\hline$\mu, K$ & $\frac{3 K-2 \mu}{3}$ & & $\frac{9 K_{\mu \mu}}{3 K+\mu}$ & $\frac{3 K-2 \mu}{2(3 K+\mu)}$ & \\
\hline$E, v$ & $\frac{v E}{(1+v)(1-2 v)}$ & $\frac{E}{2(1+v)}$ & & & $\frac{E}{3(1-2 v)}=$ \\
\hline$K, E$ & $\frac{3 K(3 K-E)}{9 K-E}$ & $\frac{3 E K}{9 K-E}$ & 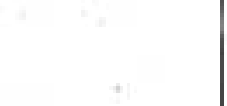 & $\frac{3 K-E}{6 K}$ & \\
\hline v, $K$ & $\frac{3 K v}{1+v}$ & $\frac{3 K(I-2 v)}{2(1+v)}$ & $3 K(1-2 v)$ & & \\
\hline
\end{tabular}

Figure 3-2. Dalley's table of constants and their conversion [37]

\subsection{Elastic Equations Using Isotropic Conditions}

The following equations substitute constants using isotropic conditions and are the general derivations of elasticity $30,37,55,53,59,119,130]$. 


\subsubsection{Strain-Stress}

Using Equation 3-13, Equation 3-15 and Equation 3-8 the strain to stress and displacement to stress equations can be written:

$$
\begin{array}{ll}
e_{x x}=\frac{1}{E}\left[\sigma_{x x}-v\left(\sigma_{y y}+\sigma_{z z}\right)\right] & e_{x y}=\gamma_{x y}=\frac{1}{\mu} \tau_{x y} \\
e_{y y}=\frac{1}{E}\left[\sigma_{y y}-v\left(\sigma_{x x}+\sigma_{z z}\right)\right] & e_{x z}=\gamma_{x z}=\frac{1}{\mu} \tau_{x z} \\
e_{z z}=\frac{1}{E}\left[\sigma_{z z}-v\left(\sigma_{x x}+\sigma_{y y}\right)\right] & e_{y z}=\gamma_{z y}=\frac{1}{\mu} \tau_{z y}
\end{array}
$$

\section{Equation 3-17 Strain - Stress relations (linear)}

\subsubsection{Displacement-Stress}

Using the linear equations and substituting Equation 3-8 in Equation 3-17 yields the displacement to stress equations below:

$$
\begin{array}{ll}
\frac{\partial u}{\partial x}=\frac{1}{E}\left[\sigma_{x x}-v\left(\sigma_{y y}+\sigma_{z z}\right)\right] & \frac{\partial u}{\partial y}+\frac{\partial v}{\partial x}=\frac{1}{\mu} \tau_{x y} \\
\frac{\partial v}{\partial y}=\frac{1}{E}\left[\sigma_{y y}-v\left(\sigma_{x x}+\sigma_{z z}\right)\right] & \frac{\partial u}{\partial z}+\frac{\partial w}{\partial x}=\frac{1}{\mu} \tau_{x z} \\
\frac{\partial w}{\partial z}=\frac{1}{E}\left[\sigma_{z z}-v\left(\sigma_{x x}+\sigma_{y y}\right)\right] & \frac{\partial v}{\partial z}+\frac{\partial w}{\partial y}=\frac{1}{\mu} \tau_{z y}
\end{array}
$$

Equation 3-18 Displacement - stress relations (linear)

\subsubsection{Stress - Strain}

30, 37, $75,53,59,119,130$

These equations inverted can be compactly written as: 


$$
\begin{array}{ll}
J_{1}=e_{x x}+e_{y y}+e_{z z} & \tau_{x y}=\mu \gamma_{x y} \\
\sigma_{x x}=\lambda J_{1}+2 \mu e_{x x} & \tau_{x z}=\mu \gamma_{x y} \\
\sigma_{y y}=\lambda J_{1}+2 \mu e_{y y} & \tau_{z y}=\mu \gamma_{x y} \\
\sigma_{z z}=\lambda J_{1}+2 \mu e_{z z} &
\end{array}
$$

or in terms of $v$ and $E$ :

$$
\begin{aligned}
& \sigma_{x x} \\
& \sigma_{y y} \\
& \sigma_{z z}
\end{aligned}=\frac{E}{(1+v)(1-2 v)}\left[\begin{array}{ccc}
(1-v) & v & v \\
v & (1-v) & v \\
v & v & (1-v)
\end{array}\right]\left\{\begin{array}{l}
e_{x x} \\
e_{y y} \\
e_{z z}
\end{array}\right\} \quad \begin{aligned}
& \tau_{x y} \\
& \tau_{x z}=\frac{E}{2(1+v)} \\
& \tau_{z y}
\end{aligned}\left\{\begin{array}{l}
\gamma_{x y} \\
\gamma_{x y} \\
\gamma_{x y}
\end{array}\right\}
$$

Equation 3-19 Linear, isotropic stress - strain relations [37]

\subsubsection{Stress-D isplacement}

When the circumstances allow, stress to displacement field equations may be written:

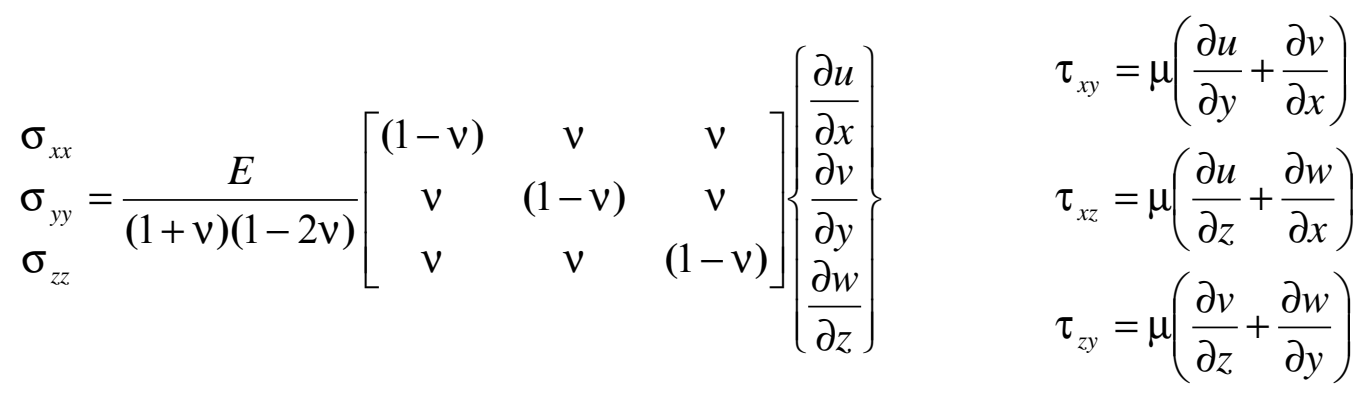

Equation 3-20 Linear, isotropic Stress - Displacement relations Equation 3-20 shows that for some problems the stress can be calculated directly from a displacement filed without first calculating the strain.

\subsubsection{D isplacement Equations of Equilibrium}

The stress displacement equations and the stress equilibrium equations can be combined to form the displacement equations of equilibrium [37] 


$$
\begin{aligned}
& \nabla^{2} u+\frac{1}{1-2 v} \frac{\partial}{\partial x}\left(\frac{\partial u}{\partial x}+\frac{\partial v}{\partial y}+\frac{\partial w}{\partial z}\right)+\frac{1}{\mu} F_{x}=0 \\
& \nabla^{2} v+\frac{1}{1-2 v} \frac{\partial}{\partial y}\left(\frac{\partial u}{\partial x}+\frac{\partial v}{\partial y}+\frac{\partial w}{\partial z}\right)+\frac{1}{\mu} F_{y}=0 \\
& \nabla^{2} w+\frac{1}{1-2 v} \frac{\partial}{\partial z}\left(\frac{\partial u}{\partial x}+\frac{\partial v}{\partial y}+\frac{\partial w}{\partial z}\right)+\frac{1}{\mu} F_{z}=0
\end{aligned}
$$

Equation 3-21 Displacement equilibrium relations [37]

In Equation 3-21, the Laplacian operator is defined as $\nabla^{2}=\frac{\partial}{\partial x}+\frac{\partial}{\partial y}+\frac{\partial}{\partial z}$ [See Mathematical Terms and Theorems in Section 3.4. This forms a displacement field similar to the stress field of Equation 3-5 and stress compatibility equations of Equation 3-23. Solving this PDE yields displacements that can be used to obtain strains, Equation 3-8 and Equation 3-6, or stresses, Equation 3-20.

\subsubsection{Compatibility}

There are six strain equations of compatibility, Equation 3-22, whose satisfaction prevents voids from opening in the body $30,37,75,53,59,119,130$. The last two strain equations of compatibility (B and C) are automatically satisfied by plane stress or plane strain field where there is no $z$ dependence [37].

The stress equations of compatibility are built up from Equation 3-22, Equation 3-5, and the stress-strain relations, Equation 3-17. The importance of these equations, Equation 3-23, is that they depend only on Poisson's ratio (in the linear, cubic case because of 
Equation 3-17). This is the reason that photoelastic experiments do not depend on Young's modulus and can model materials with different stiffnesses if Poisson's ratio is similar [37].

The material must also be simply connected and body forces must be zero for Laplace's equation, Equation 3-28 37 .

$$
\begin{array}{ll}
\frac{\partial^{2} \varepsilon_{x x}}{\partial y^{2}}+\frac{\partial^{2} \varepsilon_{y y}}{\partial x^{2}}=\frac{\partial^{2} \gamma_{x y}}{\partial x \partial y} & \text { D. } 2 \frac{\partial^{2} \varepsilon_{x x}}{\partial y \partial z}=\frac{\partial}{\partial x}\left(-\frac{\partial \gamma_{y z}}{\partial x}+\frac{\partial \gamma_{x z}}{\partial y}+\frac{\partial \gamma_{x y}}{\partial z}\right) \\
\frac{\partial^{2} \varepsilon_{z z}}{\partial y^{2}}+\frac{\partial^{2} \varepsilon_{y y}}{\partial z^{2}}=\frac{\partial^{2} \gamma_{y z}}{\partial y \partial z} & \text { E. } \quad 2 \frac{\partial^{2} \varepsilon_{y y}}{\partial x \partial z}=\frac{\partial}{\partial y}\left(\frac{\partial \gamma_{y z}}{\partial x}-\frac{\partial \gamma_{x z}}{\partial y}+\frac{\partial \gamma_{x y}}{\partial z}\right) \\
\frac{\partial^{2} \varepsilon_{x x}}{\partial z^{2}}+\frac{\partial^{2} \varepsilon_{z z}}{\partial x^{2}}=\frac{\partial^{2} \gamma_{x z}}{\partial x \partial z} & \text { F. } 2 \frac{\partial^{2} \varepsilon_{z z}}{\partial x \partial y}=\frac{\partial}{\partial z}\left(\frac{\partial \gamma_{y z}}{\partial x}+\frac{\partial \gamma_{x z}}{\partial y}-\frac{\partial \gamma_{x y}}{\partial z}\right)
\end{array}
$$

\section{Equation 3-22 Strain compatibility relations}

Compatibility Assumption requires changes at the microscale because dislocations and other defects create singularities and discontinuities in the field. 


$$
\begin{aligned}
& I_{1}=\left(\sigma_{x x}+\sigma_{y y}+\sigma_{z z}\right) \\
& \nabla^{2} \sigma_{x x}+\frac{1}{1+v} \frac{\partial^{2}}{\partial x^{2}} I_{1}=-\frac{v}{1-v}\left(\frac{\partial F_{x}}{\partial x}+\frac{\partial F_{y}}{\partial y}+\frac{\partial F_{z}}{\partial z}\right)-2 \frac{\partial F_{x}}{\partial x} \\
& \nabla^{2} \sigma_{y y}+\frac{1}{1+v} \frac{\partial^{2}}{\partial y^{2}} I_{1}=-\frac{v}{1-v}\left(\frac{\partial F_{x}}{\partial x}+\frac{\partial F_{y}}{\partial y}+\frac{\partial F_{z}}{\partial z}\right)-2 \frac{\partial F_{y}}{\partial y} \\
& \nabla^{2} \sigma_{z z}+\frac{1}{1+v} \frac{\partial^{2}}{\partial z^{2}} I_{1}=-\frac{v}{1-v}\left(\frac{\partial F_{x}}{\partial x}+\frac{\partial F_{y}}{\partial y}+\frac{\partial F_{z}}{\partial z}\right)-2 \frac{\partial F_{z}}{\partial z} \\
& \nabla^{2} \tau_{x y}+\frac{1}{1+v} \frac{\partial^{2}}{\partial x \partial y} I_{1}=-\left(\frac{\partial F_{y}}{\partial x}+\frac{\partial F_{x}}{\partial y}\right) \\
& \nabla^{2} \tau_{y z}+\frac{1}{1+v} \frac{\partial^{2}}{\partial y \partial z} I_{1}=-\left(\frac{\partial F_{z}}{\partial y}+\frac{\partial F_{y}}{\partial z}\right) \\
& \nabla^{2} \tau_{z x}+\frac{1}{1+v} \frac{\partial^{2}}{\partial x \partial z} I_{1}=-\left(\frac{\partial F_{z}}{\partial x}+\frac{\partial F_{x}}{\partial z}\right)
\end{aligned}
$$

Equation 3-23 Stress equations of compatibility [37]

Equations Equation 3-5, Equation 3-7, and Equation 3-23 combine to form:

$$
\begin{aligned}
& \nabla^{2}\left(\sigma_{x x}+\sigma_{y y}-\frac{\Omega}{1-v}\right)=0 \\
& \nabla^{2}\left(\sigma_{x x}+\sigma_{y y}\right)=\nabla^{2}\left(\frac{\Omega}{1-v}\right)
\end{aligned}
$$

\section{Equation 3-24 Laplace's equation in two-dimensions}

In two-dimensions, Equation 3-24, it is apparent that the solution depends at most, on Poisson's ratio, $\mathrm{V}$ [37]. When body forces are constant or zero, then the entire function simplifies to Laplace's equation, Equation 3-28.

\subsubsection{Potential Stress Functions}

Stresses are assumed to satisfy these stress functions or potential field equations: 


$$
\begin{array}{ll}
\sigma_{x x}=\frac{\partial^{2} \Phi_{3}}{\partial y^{2}}+\frac{\partial^{2} \Phi_{2}}{\partial z^{2}}+\Omega & \text { And in two-dimensions: } \\
\sigma_{y y}=\frac{\partial^{2} \Phi_{3}}{\partial x^{2}}+\frac{\partial^{2} \Phi_{1}}{\partial z^{2}}+\Omega & \sigma_{x x}=\frac{\partial^{2} \Phi}{\partial y^{2}}+\Omega \\
\sigma_{z z}=\frac{\partial^{2} \Phi_{2}}{\partial x^{2}}+\frac{\partial^{2} \Phi_{1}}{\partial y^{2}}+\Omega & \sigma_{y y}=\frac{\partial^{2} \Phi}{\partial x^{2}}+\Omega \\
\tau_{y x}=-\frac{\partial^{2} \Phi_{3}}{\partial y \partial x} & \tau_{y x}=-\frac{\partial^{2} \Phi}{\partial y \partial x} \\
\tau_{y z}=-\frac{\partial^{2} \Phi_{1}}{\partial y \partial z} & \\
\tau_{z x}=-\frac{\partial^{2} \Phi_{2}}{\partial z \partial x} &
\end{array}
$$

Equation 3-25 Potential field equations in three and two-dimensions [75]

\subsubsection{Bihamonic Equation}

Substituting Equation 3-25 into Equation 3-24 yields the biharmonic function [9];

$$
\begin{aligned}
& \nabla^{2}\left(\sigma_{x x}+\sigma_{y y}-\frac{\Omega}{1-v}\right)=0 \\
& \nabla^{2}\left(\frac{\partial^{2} \Phi}{\partial y^{2}}+\frac{\partial^{2} \Phi}{\partial x^{2}}\right)=\nabla^{2}\left(\frac{(1-2 v)}{1-v} \Omega\right) \\
& \nabla^{2}\left(\nabla^{2} \Phi\right)=\nabla^{4} \Phi=\nabla^{2}\left(\frac{(1-2 v)}{1-v} \Omega\right)
\end{aligned}
$$

\section{Equation 3-26 Bihamonic function of $\Phi$}

A comprehensive collection of analytically solvable mechanical engineering problems has not been compiled. It would be an asset to understanding basic engineering problems (beams, plates, pressure vessels, etc.), especially as an exact solution to compare to classical and finite element methods. 


\subsection{Mathematical Tems and Theorems}

The following terms and theorems are significant to the derivations of elasticity and potential theory.

\subsubsection{James Clerk Maxwell}

\section{Curl Definition}

$75,83,84,85,86,87,143$

Maxwell first introduced the word curl in his studies of electromagnetism [84]. Where curl F is zero then the flow is irrotational. For div F equal to zero, then the fluid is incompressible or analogously, in electromagnetics a field is solenoidal when $\operatorname{div} \mathrm{F}$ equal to zero [See divergence on page [4].

F is a gradient field; $\Phi$ is a potential function for F; and P, Q, R are components [143]. If $\mathrm{F}=\mathrm{Pi}+\mathrm{Qj}+\mathrm{Rk}$ and $\mathrm{P}, \mathrm{Q}, \mathrm{R}$ are $f(\mathrm{x}, \mathrm{y}, \mathrm{z})$, then

$$
\begin{aligned}
\operatorname{curl} \mathrm{F} & =\nabla \times \mathrm{F}=\left|\begin{array}{ccc}
i & j & k \\
\frac{\partial}{\partial x} & \frac{\partial}{\partial y} & \frac{\partial}{\partial z} \\
P & Q & R
\end{array}\right| \\
& =\left(\frac{\partial R}{\partial y}-\frac{\partial Q}{\partial z}\right) i+\left(\frac{\partial P}{\partial z}-\frac{\partial R}{\partial x}\right) j+\left(\frac{\partial Q}{\partial x}-\frac{\partial P}{\partial y}\right) k
\end{aligned}
$$

\section{Equation 3-27 Curl [143]}

\subsubsection{Saint-Venant's Relation} 24, 55, 75

Many classical elasticity problems can be solved directly in terms of stresses without using displacements by applying compatibility conditions [37]. These compatibility conditions are 
differential equations derived by Saint-Venant for strain components or integrability conditions for strain and displacements [See Equation 3-22 and 75], Saint-Venant's principle states that a system of forces acting over a small region of the boundary can be replaced by a statically equivalent system of forces without introducing appreciable changes in the distribution of stresses in regions well removed from the area of application [5].

\subsubsection{Laplace's Equation}

$14,34,77,134,137$

Pierre Simon Marquis de Laplace (1749-1827) was the first to study this group of differential equations. Laplace's equation is defined in a number of equivalent ways, which are enumerated below [143]:

$$
\begin{aligned}
& \nabla^{2} \Phi=0 \\
& \operatorname{div} \operatorname{grad} \Phi=0 \\
& \nabla \bullet \nabla \Phi=0 \\
& \Delta \Phi=0
\end{aligned}
$$

\section{Equation 3-28 Laplace's equation}

The first version will be used in any mathematical derivations, whereas the second will be used in any text explanations, "the divergence of the gradient of phi" or the Laplacian of a function. The third is the dot product of the gradient, and the final form, the del operator, is very common in physics. Equation 3-28, means that if the Laplacian is equal to zero then everywhere in the field the function is equal to its average value of neighboring points, or in other words, if u satisfies Laplace's equation then u represents a state of equilibrium [52]. 
When $\nabla^{2} \mathrm{u}$ (at a specific point) is greater than zero, then the average of neighboring points is greater than $\mathrm{u}$ where it was evaluated, and vice versa, if $\nabla^{2} \mathrm{u}$ is less than zero, $\mathrm{u}$ is less than the average. Any solutions to the Laplacian are harmonic, and solutions to $\nabla^{4} \mathrm{u}$ are biharmonic. Solutions to Laplace's equation are one of the classes of equations covered under the subject of partial differential equations $52,58,105,09,31]$

\subsubsection{Airy Stress Functions}

$12,37,86,07$

Airy stress functions are polynomial approximations of potential functions, which depend at most only on Poisson's ratio, Equation 3-24 and Equation 3-25, and are formulated to satisfy harmonic equations, Laplace's equation Equation 3-28. Airy Stress Functions are valid only to plane strain or plane stress problems, i.e. two-dimensional problems, and body forces must be zero or a scalar function of position [37]. The stress functions are valid at midpoint but only approximations at boundaries because of enforcing weak boundary conditions at the ends [See Section 4.1 Method of Solution]. Airy functions are most commonly used for beams and pressure vessel (cylindrical) problems. Airy's paper of 1862 inspired Maxwell to write his paper on reciprocal figures in1864 [75]. The method of virtual load is closely related to the reciprocal figures. Maxwell corrected a term related to shear in Airy's paper and extended the results to three-dimensions in 1870[75] Figure 3-3 shows one of the original drawings from Airy's paper of a beam in bending. 


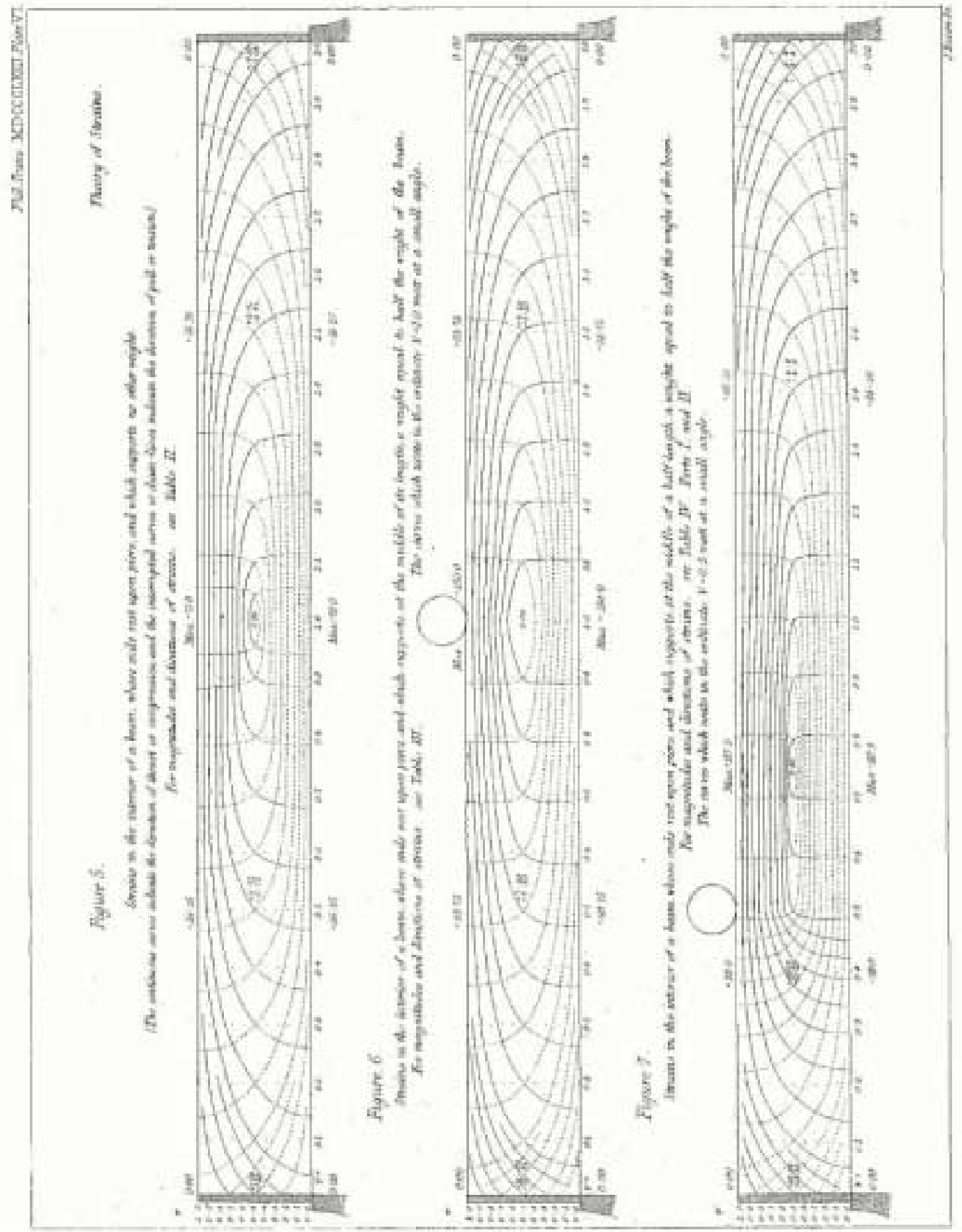

Figure 3-3. Figures of beam in bending from Airy's original treatise on stress functions. The curves are drawn from tables also included in the paper. [12] 


\subsubsection{Westergaard Complex Stress Functions}

$68,81,02,100$

In 1939, Westergaard formulated complex equations whose real and imaginary parts satisfy potential functions around mode I fracture cracks [81]. This was extended to other fracture modes, but not beyond the general vicinity of cracks. According to Pacey, et al., Westergaard equations are limited to uniform stress fields, but cracks usually grow under varying fields. This was improved upon by formulating more generally applicable potential functions [100].

\subsubsection{George Green}

Green's Theorem of 1828 is used to obtain the stress equations of equilibrium.

$$
\begin{aligned}
& \oint_{c} P d x+Q d y=\iint_{R}\left(\frac{\partial Q}{\partial x}+\frac{\partial P}{\partial y}\right) d A \\
& \oint_{c} F \cdot n d s=\iint_{R} \nabla \cdot F d A
\end{aligned}
$$

Equation 3-29 Green's theorem 143

\subsubsection{Divergence or Gauss's Theorem}

This theorem, Equation 3-30, relates the integral over a surface, S, to the divergence of a field over a volume, D.

$$
\iint_{S}(F \cdot n) d a=\iiint_{D} \nabla F d V=\iiint_{D} \operatorname{div} F d V
$$

\section{Equation 3-30 Divergence [143]}


Flux is the flow through a surface element, for a velocity field F, n normal, and da area:

$$
\text { Flux }=\iint_{S}(F \cdot n) d a
$$

\section{Equation 3-31 Flux 143}

Gauss's theorem or divergence states that the flux through a surface area is equal to the divergence of a field through a volume 75,143$]$.

\subsubsection{Stoke's Theorem}

Green's theorem in three-dimensions is Stoke's theorem:

$$
\begin{aligned}
& \oint_{c} P d x+Q d y+R d z=\iint_{R}\left(\frac{\partial Q}{\partial x}+\frac{\partial P}{\partial y}+\frac{\partial R}{\partial z}\right) d A \\
& \oint_{c} F \cdot n d r=\iint_{R}((\nabla F) \cdot n) d s
\end{aligned}
$$

\section{Equation 3-32 Stoke's theorem [143]}

Note: $\mathrm{n}$ is upper normal [See curl]

\subsubsection{Cauchy-Riemann}

The Cauchy-Riemann condition:

$$
\begin{aligned}
& \frac{\partial u}{\partial x}=\frac{\partial v}{\partial y} \text { and } \frac{\partial u}{\partial y}=-\frac{\partial v}{\partial x} \text { leads to : } \\
& \frac{\partial^{2} u}{\partial x \partial y}=-\frac{\partial^{2} v}{\partial x \partial y}
\end{aligned}
$$

Equation 3-33 The Cauchy-Riemann condition 143 
If $\mathrm{u}$ and $\mathrm{v}$ satisfy this condition, then they also satisfy Laplace's equation, Equation 3-28. To obtain an analytical solution, the problem must be well posed, the Cauchy-Riemann condition must be satisfied, and the boundary conditions must be defined. In twodimensional problems, this is always theoretically possible, because there is a unique solution for a set of boundary conditions [75]. Since the solution is a linear combination, a complicated geometry can be broken down into simple blocks and the sum of the solutions yield the total potential. Many problems have already been solved analytically, and for more complicated geometries recourse to computer programs with numerical methods and finite element modeling are currently available, though not widely used in the study of mechanical stress and strain [See Matlab's PDE toolbox and flexPDE].

The goal of the solution for three-dimensional problems is a triply orthogonal set of surfaces corresponding to the principal stresses or "isostatic surfaces" [75]. There is always a set of isostatic surfaces in two-dimensional problems [75]. Three-dimensional problems are, of course, much more difficult, and in general, the three-dimensional orthogonal set might not exist, according to Boussinesq (1872) [5].

\section{Cauchy relation:}

The Cauchy relation, $\mathrm{C} 12=\mathrm{C} 44$, is the result of isotropic assumption that the crystal is bound only by central forces [53]. Cauchy's relations led to rari-constant theory, that isotropic materials can be described by one constant with Poisson's ratio equal to 1/4 [See Figure 2-12 for comparison and references 53, 75]. Only 20\% of crystals satisfy these 
relations according to experiments preformed by Voigt (ca. 1887), and many materials are closer to $1 / 3$ rather than $1 / 4$, thus resolving an age-old argument [3]

\subsection{Nonlinear Formulation, Chaos, and Stability \\ 3.5.1 Nonlinearity \\ 94, 25, 105, 128,}

Plasticity is not the only nonlinear phenomena present in mechanics; the very difficult solution of nonlinear geometry that acts within elastic limits is applicable to structures such as nanocarbon tubes [4]. Strings, slender columns, and even the vibrations of membranes are very difficult to model using finite element programs (especially if non-linear solutions are not available). The power of Novozhilov's compact monograph on nonlinear elasticity is in the presentation of equations that reduce to classical theory when their assumptions are applied [94].

Using the nonlinear equations II.43 and II.44 from Novozhilov yields six equations with 12 unknowns. Six additional equations relating stress to displacements are formulated from strain energy relations.

These... ex press the law acoording to which the material of the given body resists various forms of deformations. A theoretical explanation of this law would necessarily require an insight into the nature of intermolecular foroes which sek to kep the partides of the solid body at definite distances from one another. The present state of scientific development, however, offers no adequate solution to this diffialt problem. [Russia in 1948]

- N ovozhilov [94] 
Thus, the importance and necessity of experimental results for specific material relationships between stress and strain is acknowledged.

Nonlinearities can be introduced into the theory of elasticity in three ways [ [4]]

- Formulas of strain components

- Equations of equilibrium of volume element

- Stress-strain relationships

This leads to four types of problems, with separate methods of simplification, in the theory of elasticity [94]:

1. Physically and geometrically linear. Example, rod extension within the limit of proportionality

2. Physically nonlinear but geometrically linear. Example, rod extension that exceeds the limit of proportionality leads to a nonlinear stress-strain relation

3. Physically linear and geometrically nonlinear. Example, bending of a thin steel strip with large angles of rotation but strains within the limit of proportionality

4. Physically and geometrically nonlinear. Example, bending of a thin steel strip with large angles of rotation and strains exceed the limit of proportionality 
Nonlinear problems of type two and four are the subjects of the theory of plasticity, type one belongs to classical or linear theory of elasticity, and type three contains elastic problems with nonlinear geometries [24, 128,

\subsubsection{Jules Henri Poincaré}

In a doctoral dissertation of 1879, Jules Henri Poincaré (1854-1912) studied general geometric properties of ordinary differential equations. He was the first to discover a chaotic deterministic problem, the three-body problem important to the stability of celestial mechanics; and his research outlined methods for the study of chaotic deterministic systems and the starting point of algebraic topology [54]. Poincaré recurrence time is important to entropy, and he understood the implications of quantum theory's difference from classical physics. He also sketched a version of special theory of relativity.

Interest in the qualitative behavior of these equations had to wait on computers that can quickly run simple programs to produce maps of qualitative behavior based on initial conditions [54]. This method was neglected until 1963 when Edward Lorenz discovered a chaotic deterministic system in a simple weather model, and Benoit Mandelbrot's work on the scientific study of fractals and fractal scaling begun in 1975 [5, 9,80$]$

\subsubsection{What is Chaos?}

18, 45, $54,74,76,79,80,101,128,138$

\footnotetext{
${ }^{6}$ This section is about a young science that crosses all disciplines and is highly philosophical in tone and content.
} 
"... you know the right equations but they're just not helpful. Y ou add up all the microsopic pieces and you... cannot extend them to the long term. They're not what's important in the problem. It completely changes what it means to Know Something." F eigenbaum [54]

An acceptable definition of chaos is not forth-coming from the sources. Wolfram and Gleick call it "a new kind of scieno" 138, 54; mathematicians call it ergodic, nondeterministic, or stochastic; engineers call it turbulence, buckling, and random. Chaos is apparent, though not fully understood, in fields like dynamical systems, nonlinear dynamics, fractals, and even cellular automata $[101,138]$. The fact that the starting conditions are not exactly known and that infinitesimally small differences in initial conditions lead, in time, to large variations in the state of the system when data becomes uncorrelated, means that the system is responding chaotically. Nearby trajectories diverge exponentially. This is one of the reasons why weather is so difficult to predict (and may be impossible) [54]. However, just because the behavior is complex, does not mean that the governing equations cannot be simple.

\section{Stability}

At resonance, amplitude increases unbounded until the system self-destructs, but when displacements become large additional physics come into play, such as nonlinear elasticity, plastic deformation, heat transfer, buckling and others [09]

Examples of equations whose chaotic nature has only recently been acknowledged:

Planetary Motion \Celestial Mechanics, Duffing, Rayleigh-Van der Pol, Lorenz, and Logistics equations [4] 
All of these equations involve derivatives, and depending on the coefficients the behavior may be chaotic and unstable, chaotic but periodic, or stable. Chaotic but periodic cycles include the weather, the movement of a pendulum (large angle), the human heart, oscillating circuits, frequency locking, and dripping faucets [54],

\section{Scaling}

[76, 79,80$]$

Scaling is a chaotic characteristic demonstrated by fractals, Julia patterns, coastline topology (fractional dimensions), and fern-like plants. Scaling is an inherent characteristic of chaos and cellular automata 79,80$]$. Properties that are scale invariant are important to selfsimilarity and understanding how scales link together to produce experimentally observable

effects. Revealing these scale invariant properties contributes to the understanding (and modeling) of properties that change with scale, like strength. An attempt is made in [142] to reveal universal scaling properties of isotropic and anisotropic fluid flows and their connection to the rate of decay of forcing functions in developed turbulence. Because chaos is universal (or mathematical) invariants in one system, bare significance to completely different systems. For example, the rate of bifurcation is a constant whether the data is from the stock market or fish populations [34].

\subsubsection{Non-uniqueness}

In the classical theory, using Hooke's law and omitting the nonlinear terms, leads to a unique solution of equilibrium 24,128 . However, reality and stability demonstrate that more than one equilibrium position may exist. 
For Maxwell, this meant a scientific instance of free will.

There are certain cases in which a material system, when it comes to a phase in which the particular path which it is describing coincides with the envelope of all such paths may either continue in the particular path or take to the envelope (which in these cases is also a possible path), and which course it takes is not determined by the forces of the system (which are the same for both cases) but when the bifurcation of path occurs, the system, ipso facto, involves some determining principle which is extra physical (but not ex tra material) to determine which of the two paths it is to follow.

W hen it is on the enveloping path it may at any instant, at its own sweet will, without ex erting any force or spending any energy, go off along that one of the particular paths which happens to ooincide with the actual condition of the system at that instant.

-J C Maxwell in a letter to Francis G alton, F ebruary 26,1879[83]

When more than one equilibrium position exists (according to Novozhilov) the classical solution is ordinarily unstable, which can have catastrophic effects when blindly applied to actual structures.

The moment of appearance of a possible bifurcation in the solution corresponds to the critical load. H enœ, two positions of equilibrium corresponding to an infinitesimal increment in the critical load differ from one another by an infinitesimal amount.

This last condition will be used as a basis for the determination of critical loads. [94]

This is observed in the theory of Euler's equations of columns, and in such real manifestations as the Tacoma Narrows bridge failure.

\footnotetext{
${ }^{7}$ Maxwell died of cancer November 5 of that same year at age 48 .
} 


\subsubsection{Potential Theory, Chaos, and Scaling}

Potential theory retains nonlinearity that is inseparable from chaos and present in ordinary and partial differential equations. If chaotic response at the microscale is the link between bulk behavior and theoretical strength at the nano-level, then the nonlinearities must be retained to preserve (and model) that behavior. The difficulty is in modeling grains under the influence of a field that has discontinuities and singularities. Part of this has been achieved in using functions like Westergaard fomplex stress functions to model cracks, and in multi-atomic models of grains [See Section 8.1 Applied Math and Modeling and references $0,42,48,00,70,63,106,112,118$.

As chaos (and cellular automata) has demonstrated, complex behavior does not necessarily mean a complex model [54, 24, 101, 128, 138. The links between each level require statistical behavior and probability. One-thousand time steps (nanoseconds) over $1000 \mathrm{~nm}$ (one-dimension) by $1000 \mathrm{~nm}$ (two-dimensions) by $1000 \mathrm{~nm}$ (three-dimensions) yields one billion cells and one trillion calculations to achieve one microsecond in one cubic micrometer of matter. This process must be repeated, one billion cells and one trillion calculations, to achieve the response of just one cubic millimeter during one second. Considering the time it would take a computer to perform these calculations, it would appear to be quicker and easier to test a specimen experimentally and let the material do the calculations naturally. This suggests that some scaling relation is present adding up all those nanoseconds to obtain discontinuities at the microscale, dislocations and grain boundaries; and they are scaled again to obtain the mostly homogenous continuum at the bulk level. 


\subsection{Denivation References}

References for general math derivations are found in [105, 119, 143]; general mechanics derivations are contained in $[30,37,75,53,59,119,130]$; and additional sources for chaos, fractals and their relation to geological and scaling phenomena can be found on the Internet. 


\subsection{Potential Field Theory Applied to Mechanics}

$22,52,68,78,00,82,109,115,131]$

The potential field theory covers many diverse areas [See also Section 7.0 enalogous systems including electromagnetic fields, mechanical stress and strain fields, geologic and gravity fields, quantum mechanics, and others $[2,68,78,00,109,131$. Potential theory is the preferential method for solving many field problems $[2,34,35,52,68,78,00,104,109$. 131, 135]. The technique involves the solution of partial differential equations (PDE) of which Laplace's equation is important for engineering applications. Diffusion and heat transfer equations can also be formulated using PDE's. Some of the equations studied in potential field theory are enumerated below:

Laplace's Equation - Elliptic PDE, a static (equilibrium) or time independent equation

Wave Equation - Hyperbolic PDE, Maxwell's electromagnetic equations

Schrödinger Equation - Second order linear PDE, basic law of quantum mechanics

Navier-Stokes Equation - Parabolic PDE, turbulence, dissipative system

Diffusion Equation - Simpler Parabolic PDE, chemical diffusion, heat conduction and random walks

Vlasov Equations - PDE Combination of electromagnetism and fluid mechanics, plasma physics, MHD (magneto-hydrodynamics) 
The Field Theory Handbook 90 ] covers Laplace's equation, Poisson equation, diffusion equation, and vector and scalar wave equations. It contains the general three-dimensional solutions for separable equations in 11 general coordinate systems illustrated in Figure 4-1, plus 29 additional systems. The approach is to transform the PDE to a coordinate system that takes advantage of symmetry in the geometry of the problem and allows for separation of variables. The use of unusual coordinate systems allows for simple description of the boundary conditions. (If the boundaries cannot be described in this manner, then the problem cannot be solved analytically.) The variables are then separated into three ordinary differential equations (ODE) that can be solved for particular solutions. The boundary conditions are applied and the unique solution can be built up from the general solutions.

For engineering applications, the potential function is usually denoted by $\varphi$, but for electromagnetic derivations, $\mathrm{V}$ denotes the potential field, and it is associated with voltage. Potential functions are designed to be harmonic functions and solutions of Laplace's equation Equation 3-28) or biharmonic and solutions to Equation 3-26. 
The eleven coordinate systems: Cylindrical

1. Rectangular $(x, y, z)$

2. Circular-cylinder $(r, \varphi, z)$

3. Elliptic-cylinder $(\eta, \varphi, z)$

4. Parabolic-cylinder $(\mu, v, z)$

\section{Rotational}

5. Spherical $(r, \theta, \varphi$,

6. Prolate spheroidal $(\eta, \theta, \varphi)$

7. Oblate spheroidal $(\eta, \theta, \varphi)$

8. Parabolic $(\mu, \nu, \varphi)$

\section{General}

9. Conical $(r, \theta, \lambda)$

$\square$ 10. Ellipsoidal $(\eta, \theta, \lambda)$

11. Paraboloidal $(\mu, v, \lambda)$
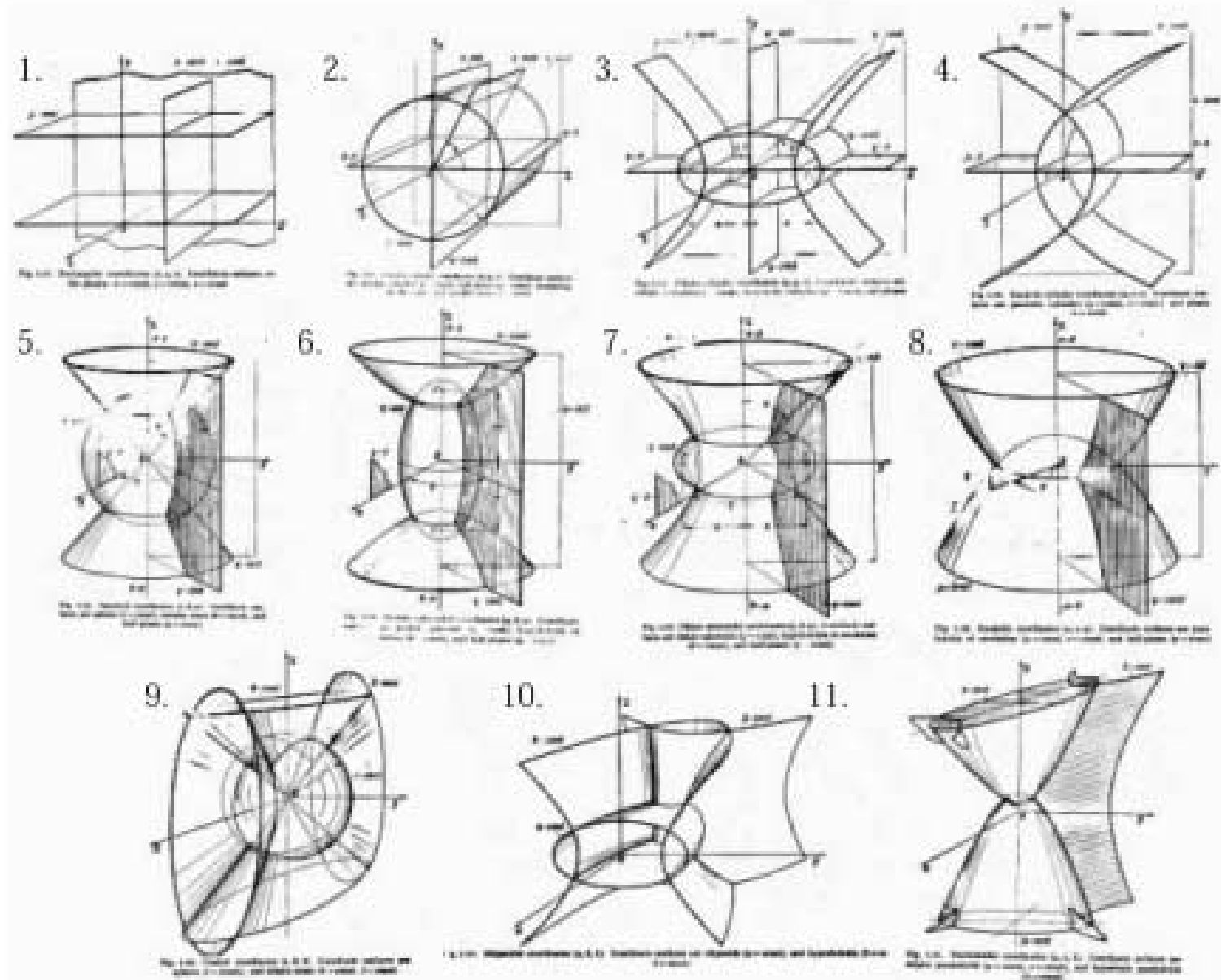

Figure 4-1. Illustration of 11 coordinate systems for solving partial differential equations [90] 


\subsection{Method of Solution}

One method for solving Laplace's equation, Equation 3-28, is by separation of variables. Many problems are separable and many coordinate systems are available. Many problems not separable require numerical solutions, but can easily be solved using PDE software. The solution is uniquely determined if either:

a) Dirichlet boundary condition - the function is specified on all boundaries, or if

b) Neuman boundary condition - the normal derivative of the function is specified on all boundaries, or both,

c) A mixed combination such that all boundaries are specified [2, $88,8,00,109,131]$,

To demonstrate this method, a simple separation in two and three variables is derived from equations Equation 4-1 to Equation 4-13. For illustrative purposes, consider a twodimensional harmonic problem, in this example the constants are applicable to a temperature distribution or an electrostatic voltage distribution on a rectangular plate. The topic of the simplest classical mechanical problem, a beam in tension, is biharmonic and contains twice as many terms and boundary conditions. A biharmonic problem in it's simplest form is the subject of Topics in the Spectral Theory of F ourth O rder E lliptic D ifferential Operators a dissertation produced in 1996 [99]. To solve the harmonic problem analytically, the solution is assumed to be in the form of a product.

Two-dimensional
Three-dimensional 
$\Phi(x, y)=X(x) Y(y) \quad \Phi(x, y, z)=X(x) Y(y) Z(z)$

\section{Equation 4-1 Separable potential function in Two-dimensional}

$\Phi$ is the potential function and it depends only on X (a function of $\mathrm{x}$ only) and Y (a function of y only) in the two-dimensional case. Applying Laplace's equation, Equation

3-28, yields the relationship between partial derivatives and these are the PDE's in Equation

Two-dimensional

$$
\begin{aligned}
& \nabla^{2} \Phi(x, y)= \\
& \frac{\partial^{2} X(x) Y(y)}{\partial x^{2}}+\frac{\partial^{2} X(x) Y(y)}{\partial y^{2}}=0
\end{aligned}
$$

Three-dimensional

$$
\begin{aligned}
& \nabla^{2} \Phi(x, y, z)= \\
& \frac{\partial^{2} \Phi}{\partial x^{2}}+\frac{\partial^{2} \Phi}{\partial y^{2}}+\frac{\partial^{2} \Phi}{\partial z^{2}}=0
\end{aligned}
$$

\section{Equation 4-2 Partial differential equations}

Evaluating and dividing by $\mathrm{X}(\mathrm{x}) \mathrm{Y}(\mathrm{y})$ :

Two-dimensional

$$
\begin{aligned}
& \frac{1}{X} \frac{d^{2} X}{d x^{2}}+\frac{1}{Y} \frac{d^{2} Y}{d y^{2}}=0 \\
& \frac{1}{X} \frac{d^{2} X}{d x^{2}}-\frac{1}{Y} \frac{d^{2} Y}{d y^{2}}=k
\end{aligned}
$$

Three-dimensional

$$
\frac{1}{X} \frac{d^{2} X}{d x^{2}}+\frac{1}{Y} \frac{d^{2} Y}{d y^{2}}+\frac{1}{Y} \frac{d^{2} Y}{d y^{2}}=0
$$

\section{Equation 4-3 0 rdinary differential equations}




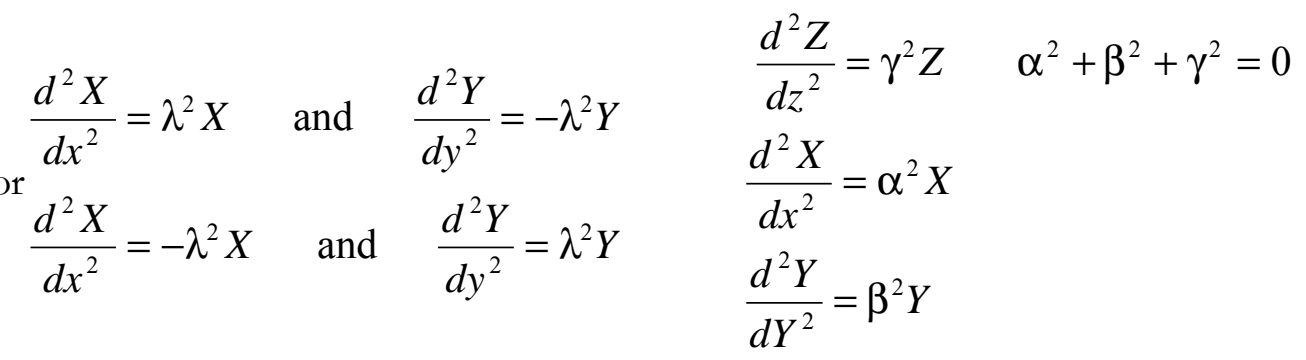

Equation 4-4 Separation of variables

Where $\alpha, \beta, \gamma$, and $\lambda$ are constants.

Equation 4-3 separates into Equation 4-4, and the boundary conditions determine which equation of Equation 4-3 applies. The solutions of these ordinary differential equations are simple. Note that the derivatives are no longer partial because each derivative is dependent on one variable only. The solutions are:

Two-dimensional

$$
\begin{aligned}
& \left.\begin{array}{l}
X(x)=A \sinh \lambda x+B \cosh \lambda x \\
Y(y)=C \sin \lambda y+D \cos \lambda y
\end{array}\right\} \text { for } \mathrm{k}=\lambda^{2} \geq 0 \\
& \text { or } \\
& \left.\begin{array}{l}
X(x)=\alpha \sin \lambda x+\beta \cos \lambda x \\
Y(y)=\gamma \sinh \lambda y+\delta \cosh \lambda y
\end{array}\right\} \text { for } \mathrm{k}=\lambda^{2}<0
\end{aligned}
$$

\section{Equation 4-5 Solutions to two-dimensional separable PDE}


Where $A, B, C, D, k, a_{1}, a_{2}, b_{1}, b_{2}, c_{1}, c_{2}$ are constants. The two-dimensional equation in Equation 4-5 is related to exponentials in Equation 4-6 through identities and Euler's formula. The three-dimensional equation is left in exponential form:

$$
\begin{aligned}
& \left.\begin{array}{c}
X(x)=c_{3} e^{\lambda x}+c_{4} e^{-\lambda x} \\
A=c_{3}+c_{4} \text { and } B=c_{3}-c_{4} \\
Y(y)=c_{1} e^{\lambda y}+c_{2} e^{-\lambda y} \\
C=c_{2}-c_{1} \text { and } D=c_{1}+c_{2}
\end{array}\right\} \text { for } \mathrm{k}=\lambda^{2} \geq 0 \\
& \text { or } \\
& \left.\begin{array}{c}
X(x)=c_{3} e^{\lambda x}+c_{4} e^{-\lambda x} \\
\alpha=c_{3}+c_{4} \text { and } \beta=c_{3}-c_{4} \\
Y(y)=c_{1} e^{\lambda y}+c_{2} e^{-\lambda y} \\
\gamma=c_{2}-c_{1} \text { and } \delta=c_{1}+c_{2}
\end{array}\right\} \text { for } \mathrm{k}=\lambda^{2}<0
\end{aligned}
$$

\section{Equation 4-6 Equivalent exponential solutions to two-dimensional}

These solutions form orthogonal sets with trigonometric equations and hyperbolic equations. The difficult part is assigning boundary conditions and solving for the constants. Where there are free surfaces, the potential is zero. This is a Neuman condition, and every surface must have defined boundary conditions (Dirichlet, Neuman, or mixed boundary conditions).

For the two-dimensional problem of a plate, when there is symmetry about the x-axis then the second solution set can be used, Equation 4-5 the left and right surfaces have zero potential, and voltage (or temperature) is applied to the top and bottom ends. If the beam were oriented horizontally with symmetry about the y-axis, then the first set of solutions applies. Solutions that display time dependent or periodic solutions should use the set that 
includes sine as a function of the dependent variable. The constants in the potential function, $A, B, C, D$, and $\lambda$, must be derived using the boundary conditions, but only four are independent.

Two-dimensional

$\Phi(x, y)=X(x) Y(y)$

$\Phi(x, y)=[A \sin \lambda x+B \cos \lambda x][C \sinh \lambda y+D \cosh \lambda y]$

Three-dimensional

$\Phi(x, y, z)=X(x) Y(y) Z(z)$

$\Phi(x, y, z)=\sum\left[a_{1}(\alpha) e^{\alpha x}+a_{2}(\alpha) e^{-\alpha x}\right]\left[b_{1}(\beta) e^{\beta y}+b_{2}(\beta) e^{-\beta y}\right]\left[c_{1}(\gamma) e^{\gamma_{z}}+c_{2}(\gamma) e^{-\gamma_{z}}\right]$

\section{Equation 4-7 Potential function}

The dimensions of the plate are $0<\mathrm{y}<\mathrm{b}$ and $0<\mathrm{x}<\mathrm{a}$. Applying the boundary condition that the left and right surfaces are at zero potential requires:

$$
\begin{array}{ll}
\Phi(0, y)=0 & \text { then } B=0 ; \\
\Phi(a, y)=0 & \text { then } \sin \lambda x=0 \\
\lambda_{m}=\frac{\mathrm{m} \pi}{\mathrm{a}} & \text { for } \mathrm{m}=1,2,3 \ldots
\end{array}
$$

\section{Equation 4-8 Applying boundary conditions to two-dimensional equations yield $B$ and $\lambda$}

The resulting potential has this form:

$$
\Phi_{m}(x, y)=\left[\sin \frac{m \pi}{a} x\right]\left[C_{m} \sinh \frac{m \pi}{a} y+D_{m} \cosh \frac{m \pi}{a} y\right]
$$




\section{Equation 4-9 Potential function}

In order to satisfy the remaining boundary conditions, all of the solutions to Equation 4-9 must be superimposed to form an infinite series. Applying the end condition, where $\mathrm{V}$ is the potential or stress at the fixed ends, yields:

$$
\begin{aligned}
& \Phi_{m}(x, y)=\sum_{m=1}^{\infty}\left[\sin \frac{m \pi}{a} x\right]\left[C_{m} \sinh \frac{m \pi}{a} y+D_{m} \cosh \frac{m \pi}{a} y\right] \\
& \Phi_{m}(x, 0)=V_{b}=\sum_{m=1}^{\infty}\left[D_{m} \sin \frac{m \pi}{a} x\right]
\end{aligned}
$$

\section{Equation 4-10 Series solution to boundary conditions}

Both sides of this equation, Equation 4-10, are multiplied by a sine function with a different index then integrated to take advantage of orthogonality and solved for D. This mathematical trick is applied again for the top boundary condition yielding a formula for $\mathrm{C}$. $\mathrm{V}_{\mathrm{b}}$ and $\mathrm{V}_{\mathrm{t}}$ can be removed from the integration when they are not functions of $\mathrm{x}$ or $\mathrm{y}$, respectively.

$$
\begin{aligned}
& D_{m}=\frac{2 V_{b}}{m \pi}\left[1-(-1)^{m}\right] \\
& \Phi_{m}(x, b)=V_{t}=\sum_{m=1}^{\infty}\left[\sin \frac{m \pi}{a} x\right]\left[C_{m} \sinh \frac{m \pi}{a} b+D_{m} \cosh \frac{m \pi}{a} b\right] \\
& C_{m}=\frac{D_{m}}{\sinh \frac{m \pi}{a} b}\left[\frac{V_{t}}{V_{b}}-\cosh \frac{m \pi}{a} b\right]
\end{aligned}
$$


The final analytical solution is Equation 4-12, which can be easily programmed or solved numerically.

$$
\begin{aligned}
& \Phi(x, y)= \\
& \sum_{m=1}^{\infty} \frac{2 V_{b}}{m \pi}\left[1-(-1)^{m}\right]\left[\sin \frac{m \pi}{a} x\right]\left\{\left[\frac{V_{t}}{V_{b}}-\cosh \frac{m \pi}{a} b\right] \frac{\sinh \frac{m \pi}{a} y}{\sinh \frac{m \pi}{a} b}+\cosh \frac{m \pi}{a} y\right\}
\end{aligned}
$$

\section{Equation 4-12 Final form of potential function}

Equations Equation 4-1 to Equation 4-12 are taken directly from electrostatics field derivations from lecture notes for an undergraduate physics electromagnetics laboratory and reference [148]. Additional sources for this derivation can be found in $9,135,82,52$, 131, and 105]. This potential distribution is displayed in the Matlab model below, Figure 4-2.

Equation 4-12 was solved for the potential field using the following substitutions: the $\mathrm{x}$ and $\mathrm{y}$ axis are arbitrary units of length; the dimensions set to one by one $\left(\mathrm{a}=\mathrm{b}=1\right.$ unit); $\mathrm{V}_{\mathrm{b}}$ and $\mathrm{V}_{\mathrm{t}}$ are set to 100; and the left and right sides are at zero potential. The scale is in potential units, which are volts for electrostatic problems, temperature for heat diffusion problems, and stress for mechanical problems. However, the particular boundary conditions of this example do not correspond to a physically possible load condition. The potential scale may be interpreted also as a percentage because of the choice of values. 


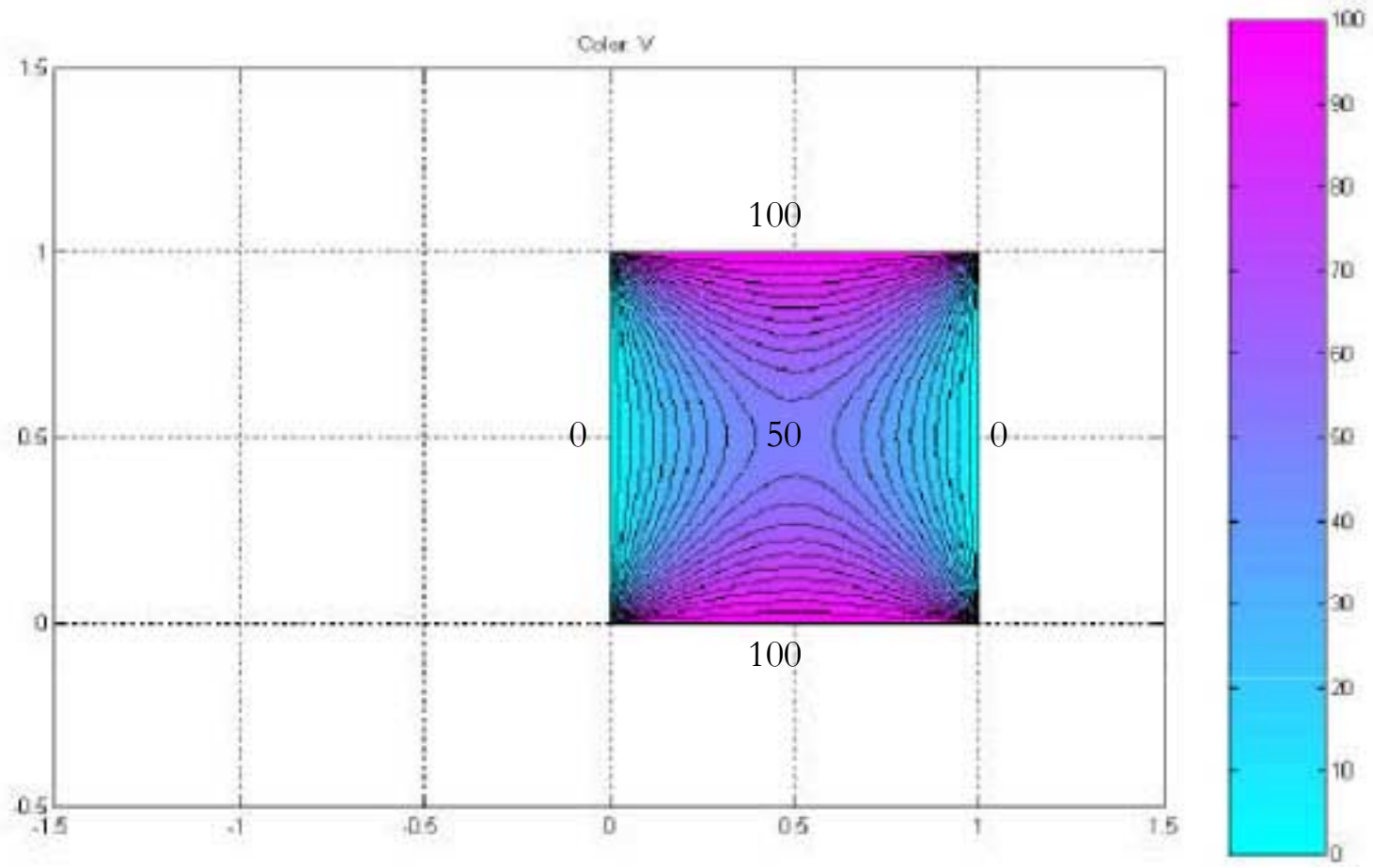

Figure 4-2. Electrostatic potential derived using potential fields and plotted in Matlab PDE toolbox

If the derived potential was for the biharmonic mechanical stress, then to solve for stresses, Equation 3-25 is used, and the derivatives of the potential function yield the stresses. Because the Laplacian of the voltage is equal to zero and the Laplacian of mechanical stresses is also zero, there is a direct relationship between the first invariant $\left(\mathrm{I}_{1}\right)$ and voltage. They are proportional and for this reason, voltage is used to produce isopachic lines that can be related to average stress or to the invariant $\left(\mathrm{I}_{1}\right)$ [See also 4.3 .1 Photoelastic Theory and 7.0 Analogous Systems].

Westergaard Complex Stress Functions in Section 3.4.5 make use of the orthogonality of complex equations to obtain separable solutions to Laplace's equation, Equation 3-28 81]. 
These equations are applied to the area near and around cracks and have been formulated for the particular modes of failure, but not for entire structures like Airy stress functions [See Section 3.4.4 Airy Stress Functions]. Martell [31] contains derivations and equations with comparisons for reference, and Figure 4-4 graphically represents the agreement between experiment and simulation based on Westergaard-based solutions.

The complexity of Equation 4-12 explains why these equations are not generally used for bulk mechanical calculations, but many computer programs including Matlab's PDE toolbox, Mathematica, and PDE solvers like flexPDE, offer numerical solutions to potential field equations overlaid on a familiar finite element grid [See Section 8.0 Advances in Computation and Material Science. Because potential fields are so prevalent in nature, many of these programs are cross-discipline and offer solutions to many specialized problems [See Section 7.0 Analogous Systems].

\subsection{Spherical Harmonics and Legendre Polynomials}

The study of spherical harmonics began in 1782 in a paper by Legendre on zonal harmonics (1785 See bibliography in 78, which inspired Laplace’s paper dealing with general spherical harmonics and the theory of the potential [78, 84]. Spherical harmonics are the series solutions for potential field problems with spherical symmetry and are covered in undergraduate physics, electrostatics [135]. Spherical harmonics were applied to the theory of gases by Maxwell in 1879, which was highly regarded by Boltzman whose pioneering work in kinetic theory led to statistical mechanics 83, 103. Spherical harmonics are 
expanded in Legendre polynomials, also known as zonal harmonics, and are the solutions to the Legendre wave equation [135, 104,

Legendre polynomials are the expansion to the series solution of separable equations in spherical coordinates. The general equation is:

$$
\phi(r, \theta)=\sum_{l=0}^{\infty}\left(A_{l} r^{l}+B_{l} r^{-(l+1)}\right) P_{l}(\cos \theta)
$$

\section{Equation 4-13 General solution to axially symmetric separable equations in spherical coordinates [135]}

Where $P_{l}(\cos \theta)$ is the Legendre polynomial and the first 3 terms are listed below with the recursive formula for generating the polynomials.

$$
\begin{array}{ll}
P_{0}(\cos \theta)=1 & \begin{array}{l}
\text { Recursive formula } \\
(l+1) P_{l+1}(\cos \theta)=(2 l+1)(\cos \theta) P_{l}(\cos \theta)-l P_{l-1}(\cos \theta)
\end{array} \\
P_{1}(\cos \theta)=\cos \theta & \text { A and B are constants. The variables are r, radius, and } \theta, \\
P_{2}(\cos \theta)=1 / 2(3 \cos \theta-1) & \text { angle, and the indices are } l \text { and } i .
\end{array}
$$

Equation 4-14 Recursive formula and first three Legendre polynomials [135] Using Legendre polynomials or spherical harmonics as an interpolative tool yield interesting results that can have increased accuracy depending on the terms retained. Legendre polynomials are employed for post-processing finite element results. According to [141, using Legendre polynomials produces better point-wise stress extraction by the complementary energy principle (SEC) when the exact FEM solution is not smooth. Bessel functions are well known to engineering even if their source is unfamiliar. Solutions to 
differential equations in cylindrical coordinates can be expressed in terms of Bessel functions $[22,68,68,80,109,131]$

Spherical harmonics are also used to solve Schrödinger's wave equation [See Section 7.2 Quantum Analogy to obtain the shells of atomic theory and the field density describing where electrons are likely to be found 104,135. Atomic simulations of atoms, crystals, and grains are constructed using potential theory $2,8,20,31,49,60,63,102,118$. This means that quantum mechanics is also an application of potential field theory [See Section 7.2 Quantum Analogy.

\subsection{Photoelastic Fields}

An experimental realization of potential fields is produced using photoelastic materials. Stress analysis using photoelasticity is a well-developed experimental method for determining the state of stress at a point based on fringe numbers utilizing isochromatic and isoclinic information. Many advances in technique, apparatus, and numerical methods are responsible for continued interest in photoelastic testing [61, 126, 139]. However, potential theory can dramatically improve upon the accuracy obtained by photoelastic methods. Comparison with photoelastic results demonstrates a qualitative experimental verification of potential theory models [See Eigure 4-3 and Eigure 4-4]

Figure 4-3 demonstrates the characteristic photoelastic pattern generated at a crack tip. The similarities to the dipole illustrated in Figure 4-4 (right) are unmistakable. PDE Software used to generate the dipole pattern can also be used to generate the stress pattern allowing 
for better numerical extraction at specified points. The numerical values can be compared to photoelastic results for correlation with experimental data. Note the simulation in Figure 4-4, which shows an excellent qualitative correlation to the actual photoelastic experiments of crack tips. The potential functions used in this particular simulation are similar to Westergaard stress functions [44],

PACEY M.N., PATTERSON E.A., JAMES M.N.

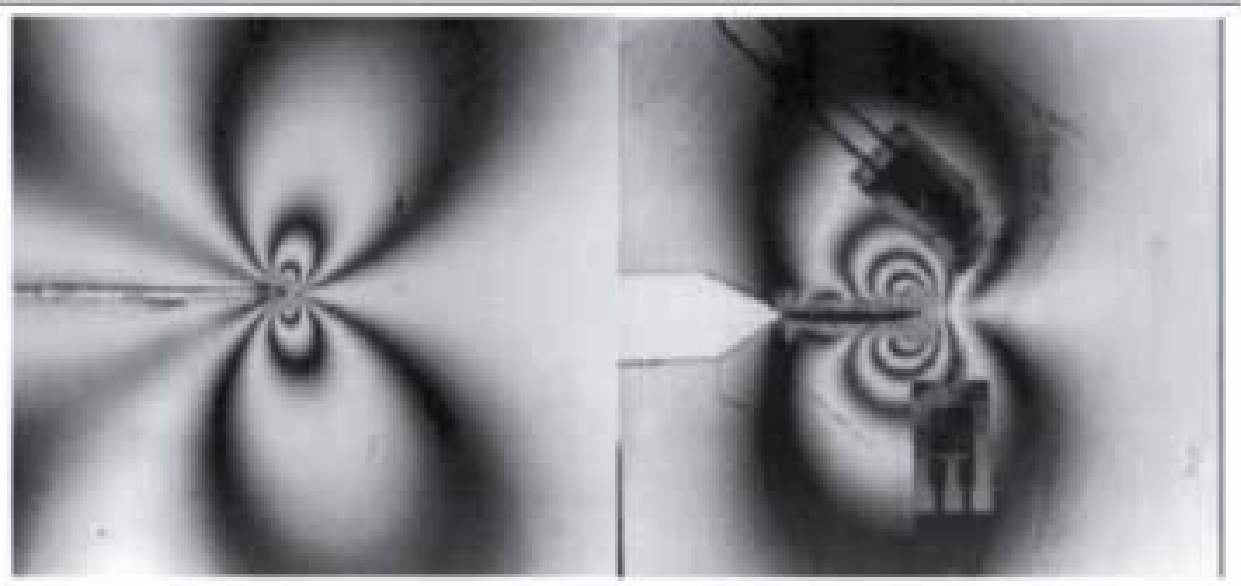

Phosographs showing examples of (left) the fringe pattern near a notch tip and (right) the fringe pattem near a crack tip, both under mode I loading

Figure 4-3. Photoelastic response at a crack tip [100] 


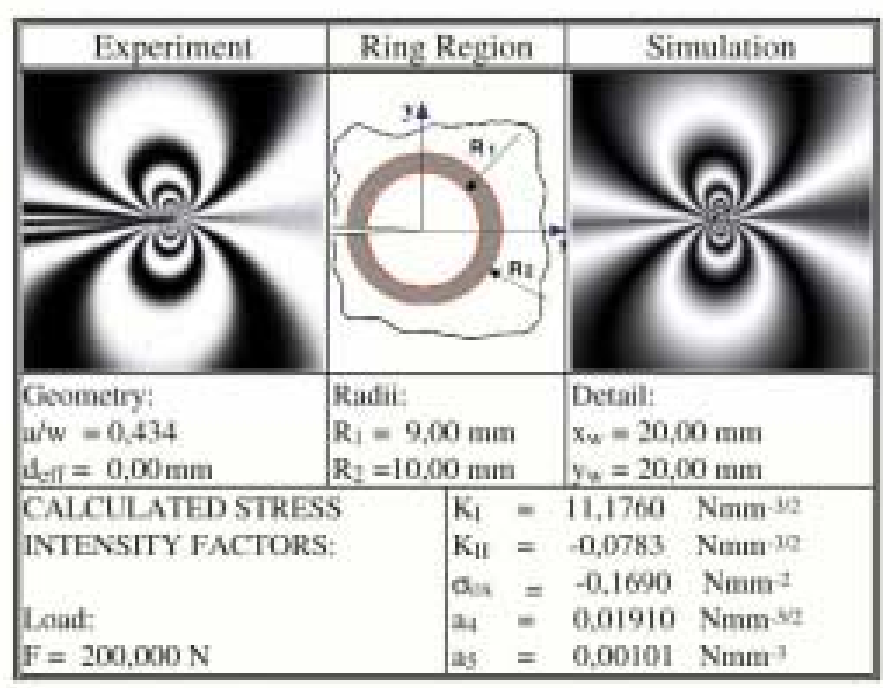

Photoelastic determination of stress intensity factors by using the 5-parameter method

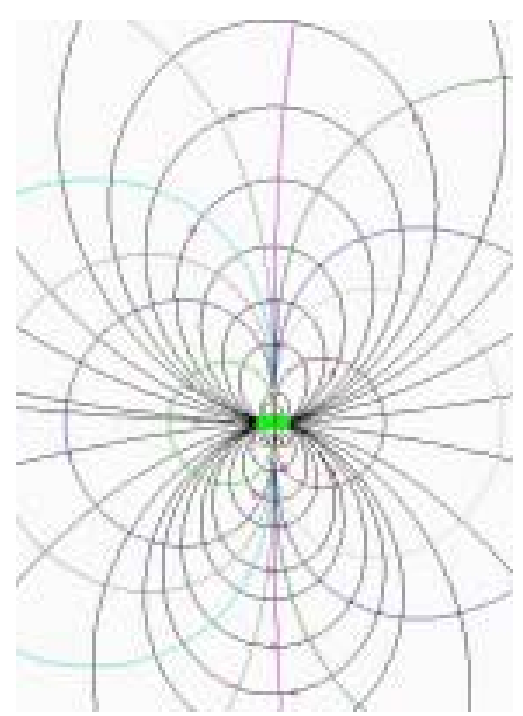

Electrostatic Dipole

Figure 4-4. Photoelastic expeniment and VIP simulation of crack tip compared with dipole (right) generated with Equipotential 3.1 software [44]

\subsubsection{Photoelastic Theory}

29, 30, 37, 44, 59, 64, $71,72,100,126,136$

The experimental stress analysis method of photoelasticity allows one to visualize the

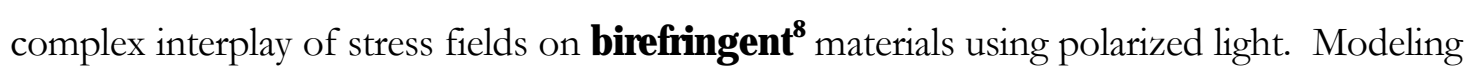
of complex parts and structures is possible using these materials [37]. The results are applicable to common engineering materials under specific circumstances and conditions, even though the test material and the building material may have different material property constants [37. By matching Poisson's ratio, the photoelastic material can be expected to

\footnotetext{
${ }^{8}$ Birefringent materials allow light to pass through the material at different speeds depending on the orientation of the sample.
} 
respond like the building material [37]. This is also the result obtained from the stress equations of compatibility, Equation 3-23.

The equations used to extract stresses from photoelastic data are $30,37,75,53,59,119$. 130]:

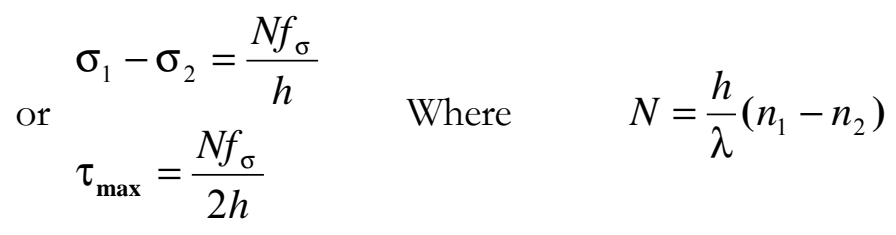

Equation 4-15 Photoelastic stress extraction equations

The difference in principal stresses (or 2 times the maximum in-plane shear stress) is determined from the number of fringes $(\mathrm{N})$, divided by the thickness ( $\mathrm{h}$ ) of the model, and multiplied by a constant factor $\left(f_{\sigma}\right)$ determined by calibrating the model material. The color of the fringe comes into the equation in the definition of $\mathrm{N} ; \lambda$ is the wavelength of light and the indices of refraction (n) are associated with the principle stress directions.

However, these equations only describe how the light is affected by stress in a birefringent material. They are not the equations of interest for modeling or mathematically describing the pattern. Potential field theory is needed for the mathematical model.

The visual similarities to electromagnetic fields should be apparent, and occur because electrostatics must also satisfy Laplace's equation, Equation 3-28 [135], This similarity is useful for another two-dimensional experimental technique in which conductive, Teledeltos 
paper is cut to the same geometry as that of the test specimen [37. A voltage is applied to the edges at an electrical potential to simulate the mechanical stress potential, and a complete circuit is made to the ground, corresponding to the end conditions of a mechanical system [37]. By probing anywhere within the model, a voltage is obtained that is proportional to the average stress at that point 37 . Lines of constant voltage are isopachic lines, where the sum of the two principle stresses is constant [37]. These are the equipotential lines of electrostatics [135]

Photoelasticity has experienced a resurgence of interest due to a reduction in the cost of equipment and better computer imaging software [61, 10, 136, 139, The results are more realistic than finite element software solutions with less cost in terms of computer time and man-hours spent programming, especially for complicated geometries, but there is a limit to the accuracy that can be expected from this method, even using sophisticated computer image capture, processing, and advanced photoelastic testing equipment. One of the difficulties in interpreting photoelastic results lies in knowing which fringes, located in

different parts of the model, are of equivalent stresses [37, 139]. New combined methods using thermoelastic and photoelastic data still have errors ranging from $10 \%$ to more than $30 \%$ and without good resolution [139].

\subsubsection{Comparison}

The process of performing a photoelastic experiment and interpreting the results has been described as part art and part science 61]. The incredible work by Thamm, Figure 4-5 and Figure 4-6, where the lines of stress and force were hand drawn over photoelastic results is 
an example. The goals of photoelasticity can be modeled more accurately using PDE software. The ability to model a part, visualize the flow, and then easily make adjustments like cutting holes or adding components to the model, is indispensable as a design tool and very instructive to the student learning about the "flow of stress" 37 126. Photoelastic models cannot be easily modified, and the material ages badly, creating stress concentrations at points where excess humidity is absorbed into the model [37] 


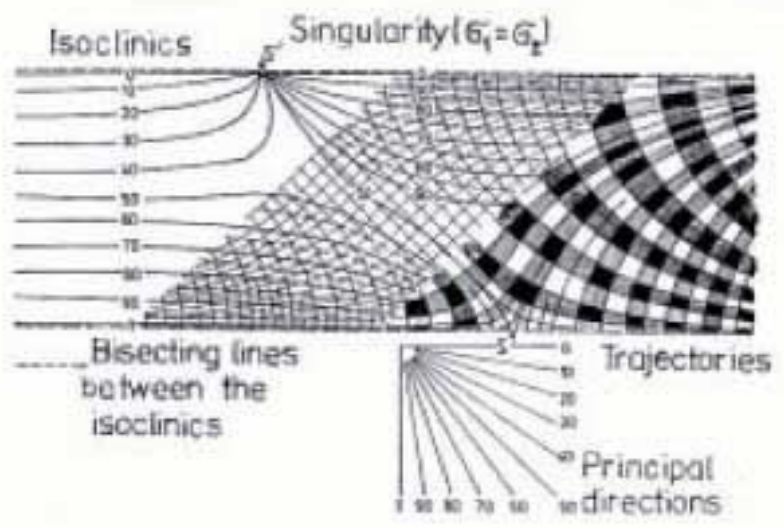

Drawing the stress trajectories from an isoclinic pattern of plane photoelasticity

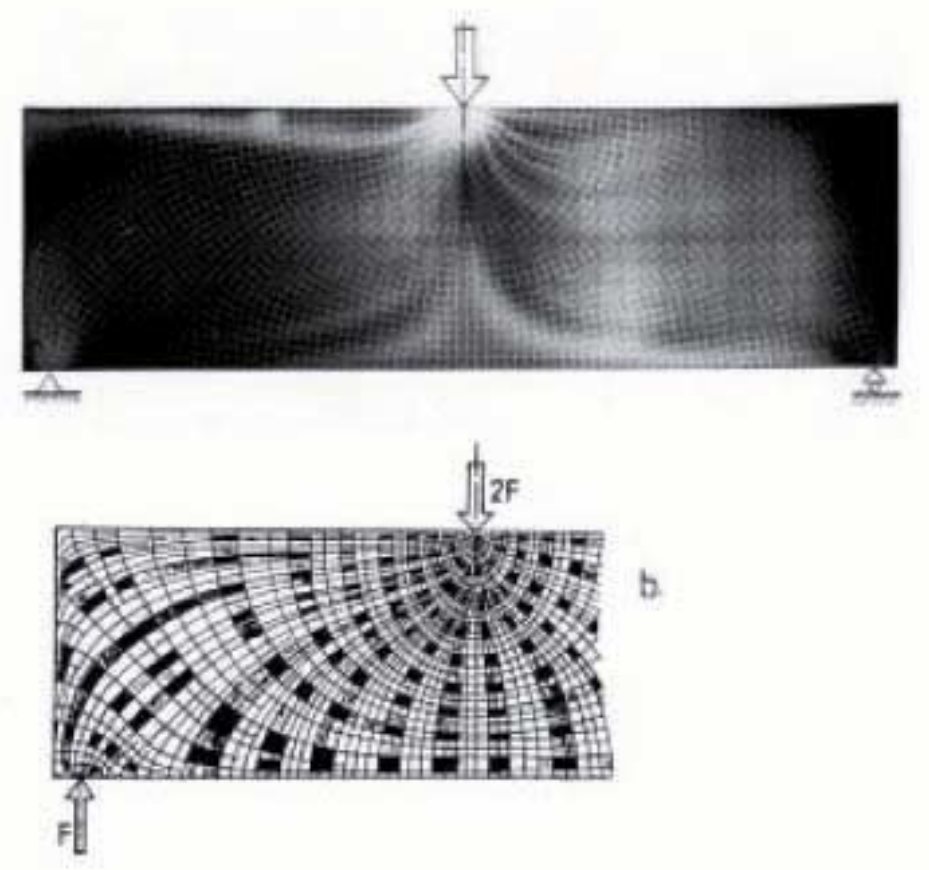

Pattern of stress trajectories of a short beam in bending. a. derived optically, b. drawn by hand

Figure 4-5. Thamm's hand drawn stress fields for component design, beam in bending 126] 

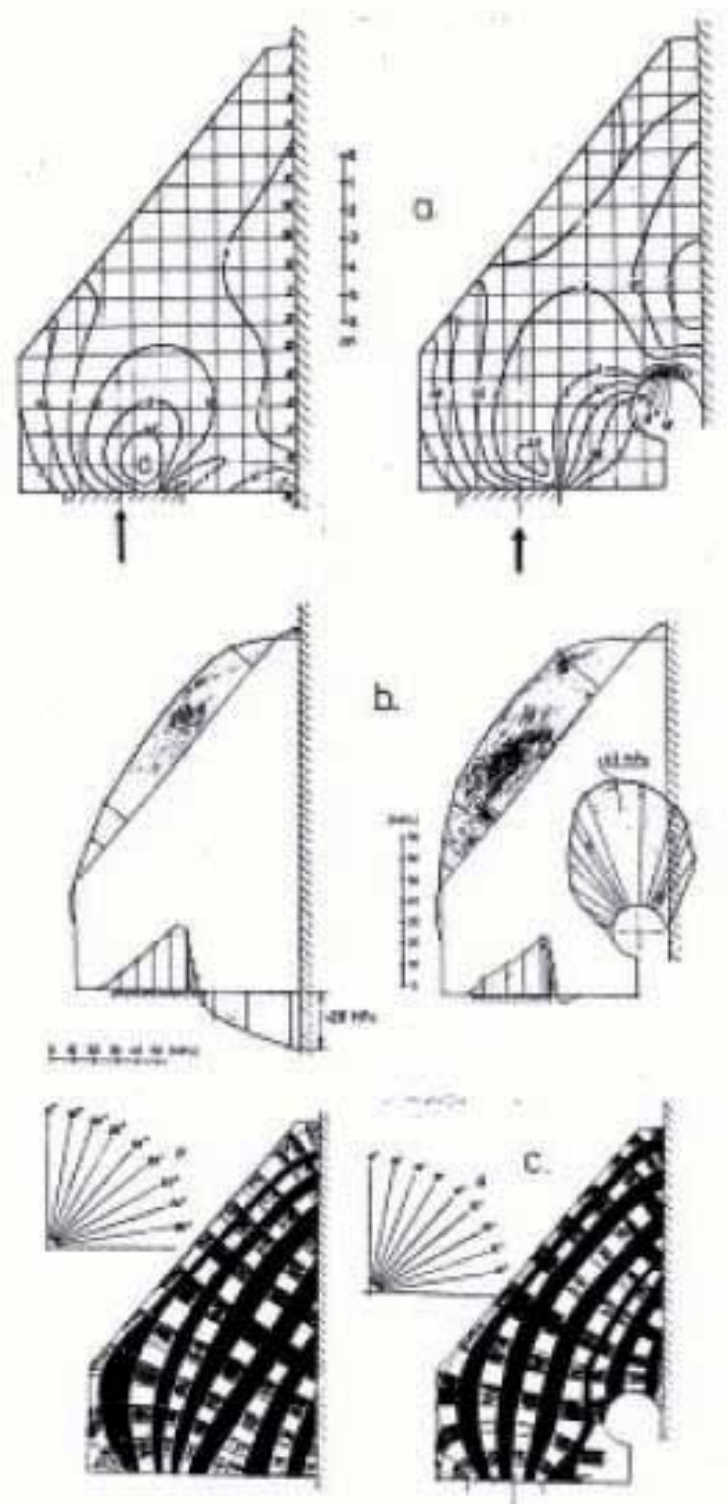

Results of the photoelastic investigation of the model shown in Fig. 7. a isochromatic fringe pattem. b. boundary stress distribution. $\mathrm{c}$. the set of stress trajectories.

Figure 4-6. Thamm's hand drawn stress fields for engine component design [126] 


\begin{tabular}{|ccc|}
\hline Potential Theory & $\underline{\text { Photoelastic Theory }}$ \\
\hline Isostatics & $\equiv$ & Isochromatics \\
$\perp$ & & $\perp$ \\
Lines of Force & $\equiv$ & Isoclinics \\
\hline
\end{tabular}

Figure 4-7. Diagram of the comparison between potential theory and photoelastic theory

In potential theory, Figure 4-7, isostatics are lines of constant stress and so are isochromatics. Isostatics are perpendicular to the flow of the force lines as isoclinics are perpendicular to the isochromatics. The lines of force are indistinguishable from isoclinics.

Because potential theory models create fields virtually identical to the delicate photoelastic patterns, many of the same arguments for using photoelastic techniques are applicable to potential theory models without the complications of experimental measurements.

Many of the reasons for not developing potential theory into an aid for mechanical stress/strain analysis have been eliminated in recent years.

\section{Old Problems}

- Difficult Math-Elliptic integrals

- No closed form solutions

- Infinite series

- Inseparable variables 
- Difficulty generalizing results

New Solutions

- Handbooks

- Advances in potential theory in related sciences:

Geology [81, 110], Electromagnetics, Quantum physics, Vibrations and Acoustics, Gravity [35], and Math

- Numerical Methods-

Computers, Parallel processing, Finite elements, Chaos and nonlinear solutions

Note that potential theory has a long history of being used an aid for fluids, electromagnetics, quantum mechanics, geology, and other sciences. [See Section 7.0 \begin{tabular}{|l|l|l|}
\hline Analogous Systems and Section & .0. Advances in Computation and Material Science \\
\hline
\end{tabular} 


\subsection{Model of Beam Using PDE}

The following models were created using Matlab's PDE toolbox; this allows Matlab's powerful numerical solvers to calculate the series solutions. An arbitrary aspect ratio of four and arbitrary loading is used to produce clear field patterns. The beam being modeled is under simple tension that produces a constant stress field according to classical theory; therefore any deviation from a constant field will be clearly visible.

The classical model is the typical free-body diagram of a beam in tension; both ends are free and the load produces a constant stress (force by area) throughout the model. There is deflection due to the force equal to the strain (Young's modulus by stress) distributed over the length of the beam and there is contraction through the width, Equation 5-1. The assigned constants and calculated values are noted below.

$$
\begin{array}{ll}
E=1000 \quad v=0.3 & \text { Beam } 0.5 \times 2.0 \\
\sigma_{y y}=\frac{P}{A}=100 & \varepsilon_{\mathrm{y}}=\frac{\sigma_{y y}}{E}=0.1 \\
\sigma_{x x}=0 & \varepsilon_{\mathrm{x}}=-v \frac{\sigma_{y y}}{E}=-0.003 \\
\tau_{x y}=0 & \delta=\frac{P L}{A E}, \quad \delta_{y}=0.2, \quad \delta_{x}=-0.0015
\end{array}
$$

\section{Equation 5-1Classical mechanical equations for a beam in tension with assigned constants and calculated values}

Modeling a free-body diagram is difficult because it is capable of rigid body translation and

rotation. To simulate the beam in tension, two Matlab models are described with different 
end conditions or boundary conditions and the subtle differences are exposed. A third model with similar deflection but different boundary conditions and entirely different potential field is modeled to demonstrate the difficulties in assigning boundary conditions and their effects. The unstressed model is also shown for comparison.

The Structural-Mechanics/Plane Stress application is chosen for this model from the choices of: Generic Scalar, Generic System, Structural Mechanics/Plane Stress, Structural Mechanics/Plane Strain, Electrostatics, Magnetostatics, AC Power Electromagnetics, Conductive Media DC, Heat Transfer, or Diffusion. All of these problems are solved using PDE's with application specific variables and graphs. The equation solved by Matlab is:

The boundary conditions for the Matlab models and equations are:

\section{Dinichlet-}

$h * u=r$

$\left[\begin{array}{ll}h 11 & h 12 \\ h 21 & h 22\end{array}\right] u 2=\begin{aligned} & r 1 \\ & r 2\end{aligned}$

Weight $-h$

Potential $-u$

Displacement $-r$

Fixed end condition:

$\left[\begin{array}{ll}1 & 0 \\ 0 & 1\end{array}\right] u 1=\begin{aligned} & 0 \\ & u 2\end{aligned}=$

\section{Neuman-}

$$
\begin{aligned}
& n * c * \operatorname{grad}(u)+q * u=g \\
& n * c * \nabla\left(\begin{array}{l}
u 1 \\
u 2
\end{array}\right)+\left[\begin{array}{ll}
q 11 & q 12 \\
q 21 & q 22
\end{array}\right] u 2 \\
& u 2
\end{aligned}=\begin{aligned}
& g 1 \\
& g 2
\end{aligned}
$$

Normal $-n$ 
Potential- $u$

Surface tractions - $g$

Free surface boundary condition:

$g=q=0$

Traction across surface:

$g 1=\sigma_{x x}=0$

$g 2=\sigma_{y y}=100$

$c=\left[\begin{array}{cccc}2 G+\mu & 0 & 0 & \mu \\ 0 & G & G & 0 \\ 0 & G & \mathrm{G} & 0 \\ \mu & 0 & 0 & 2 G+\mu\end{array}\right]$

In the following models, the first beam is classically solved, the second is without applied stress, the third follows Dirichlet conditions where the displacement is prescribed on the bottom, and the fourth follows Neuman conditions with stress prescribed on the bottom. All four models are shown in each graph. The deformed models are not to scale, but are exaggerated. Matlab uses a finite element framework with triangular elements for the numerical solution of the PDE. These equations are different from typical finite element packages like Ansys or Abaqus, though they can be modified to perform the necessary operations. 


\subsection{Results of Comparison}

These results demonstrate the character and structure of potential field solutions even in the simplest classical model of a beam in tension. This method justifies simplifying assumptions by the good agreement to the classical model. It shows interesting results of Saint-Venant's principle, which are the reasons for not focusing too closely on where the loads are applied.

In Figure 6-1, the absolute displacement is calculated and the graph shows that at the fixed ends, the isostatic surfaces curve away from the ends. The fixed ends maintain their original width and taper to approximately the same width as the classical model.

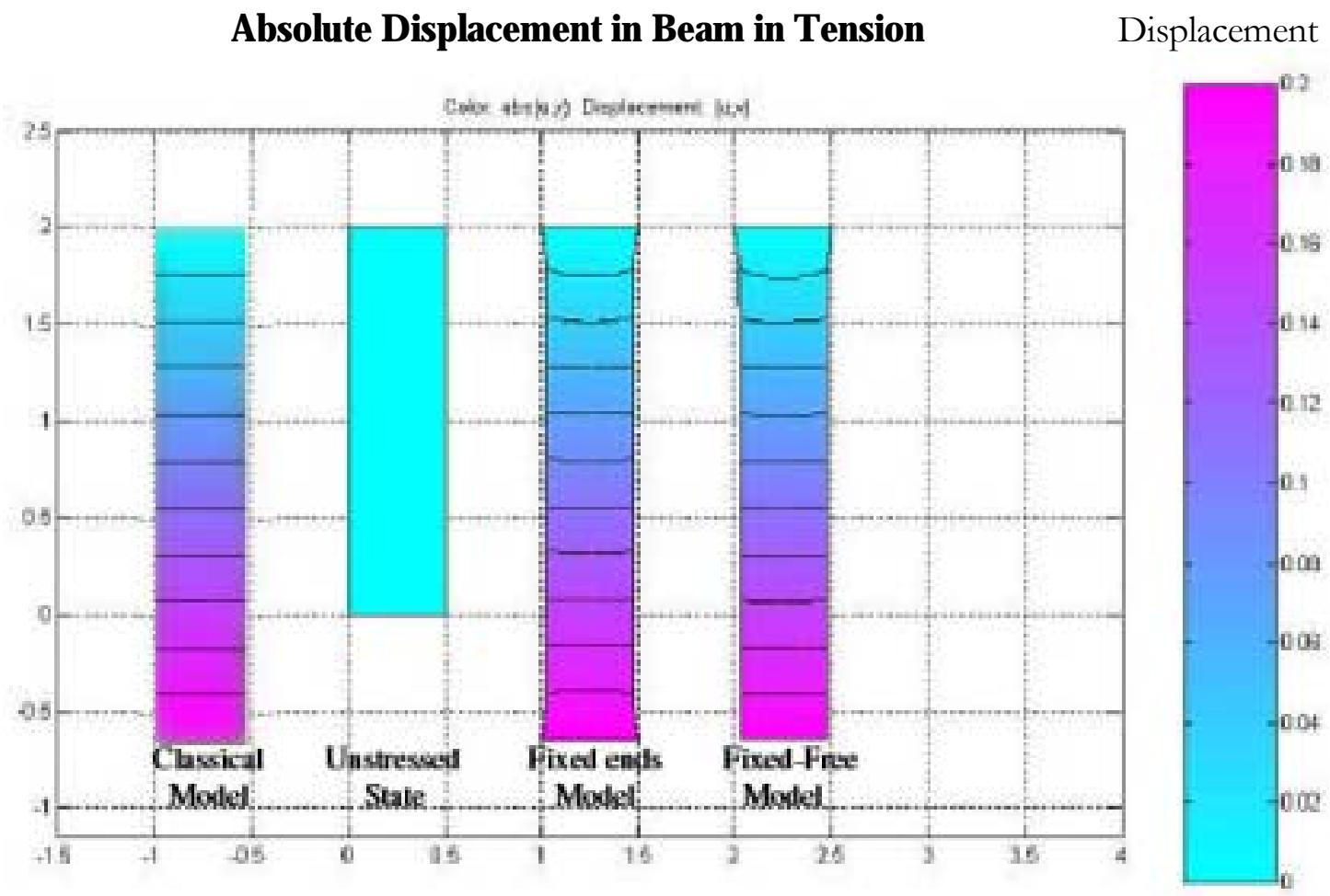

Figure 6-1. Absolute displacement of a beam in tension 
The X-displacement of Figure 6-2 shows clearly how the fixed ends affect the flow. Note that the $\mathrm{x}$-displacement is 100 times smaller than the displacement in the direction of the force and has a very small contribution to the total field. Symmetry is apparent about the vertical centerline of the beam.

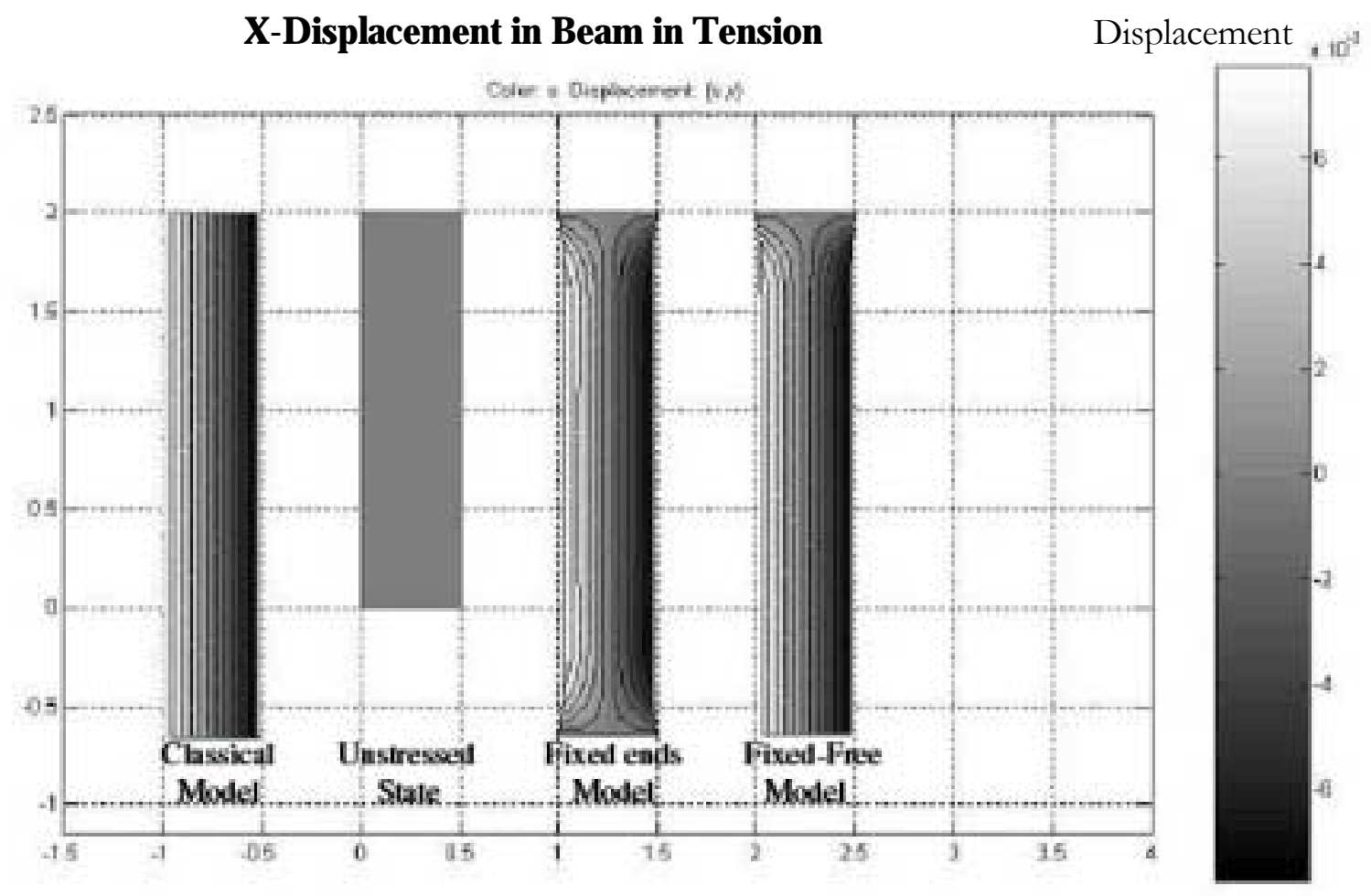

Figure 6-2. X-displacement of a beam in tension 
Figure 6-3 shows the Von Mises stress of the beam, which is simply constant for the classical model. The color-coding allows for greater resolution of the isostatic surfaces and is reminiscent of photoelastic models. The color could be coded to photoelastic data producing purely computational, photoelastic-like fringes to compare to real models.

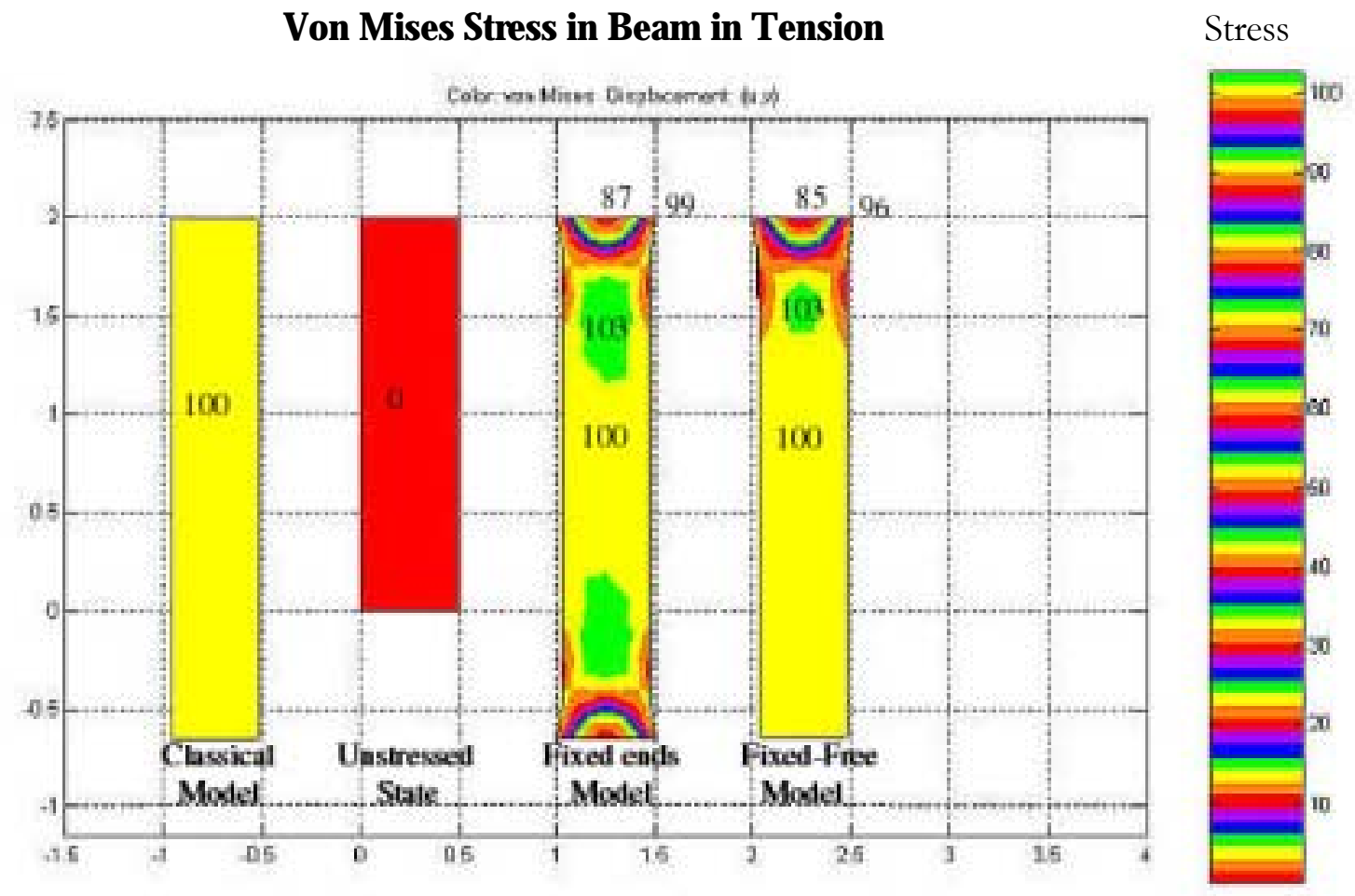

Figure 6-3. Von Mises Stress of a beam in tension

Most of the model, Figure 6-3, is in a state of constant stress of $100 \%$ of the applied stress. It is interesting to note that the stress actually drops at the fixed ends, and a stress concentration is located at the surface labeled 103 extending almost a third of the model for fixed ends and one quarter into the fixed-free model. 
The first principal stress in the classical model is zero (stress in the x-direction). The maximum attained in the PDE models is $25 \%$ applied stress and occurs at the fixed ends, Figure 6-4. The fixed ends prevent the beam from relaxing in the x-direction causing stress, whereas the center of the beam experiences only strain.

\section{Principal Stress 1 in Beam in Tension}

Stress

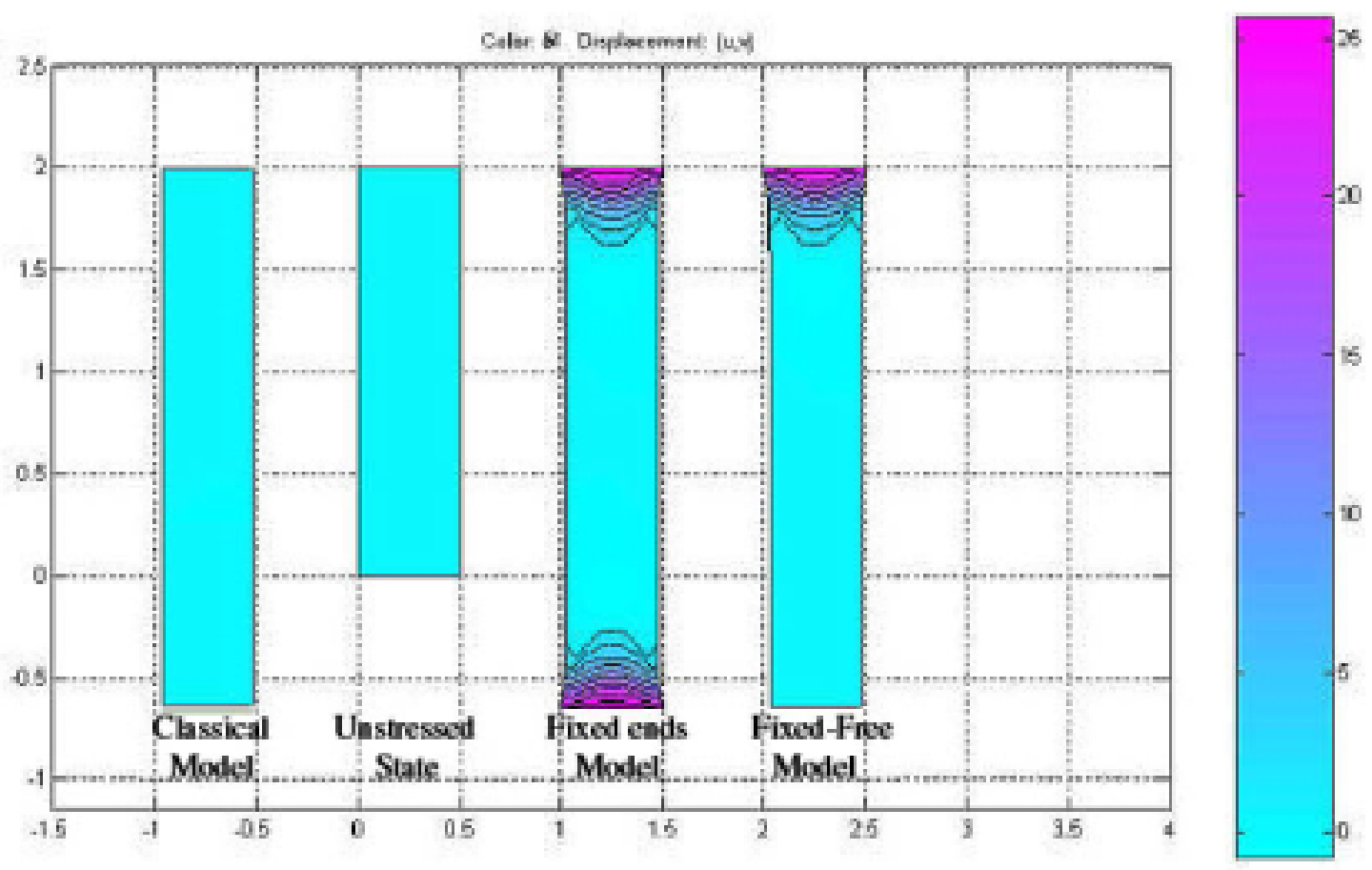

Figure 6-4. First principal stress of a beam in tension

The second principal stress in the classical model is stress in the y-direction. The Figure 6-5

shows the expected stress concentration at the corners of the fixed ends. In this figure there is also a small reduction of stress at the center of the fixed ends and a small stress concentration less than one quarter from the fixed ends on both models. 
The fixed-fixed model has a significantly higher corner concentration and the fixed-free model shows lower stresses at top center and more pronounced lower stresses along the sides near the quarter mark. Again, the color scheme was chosen to simulate photoelasticity and highlight the isostatic surfaces.

Principal Stress 2 in Beam in Tension
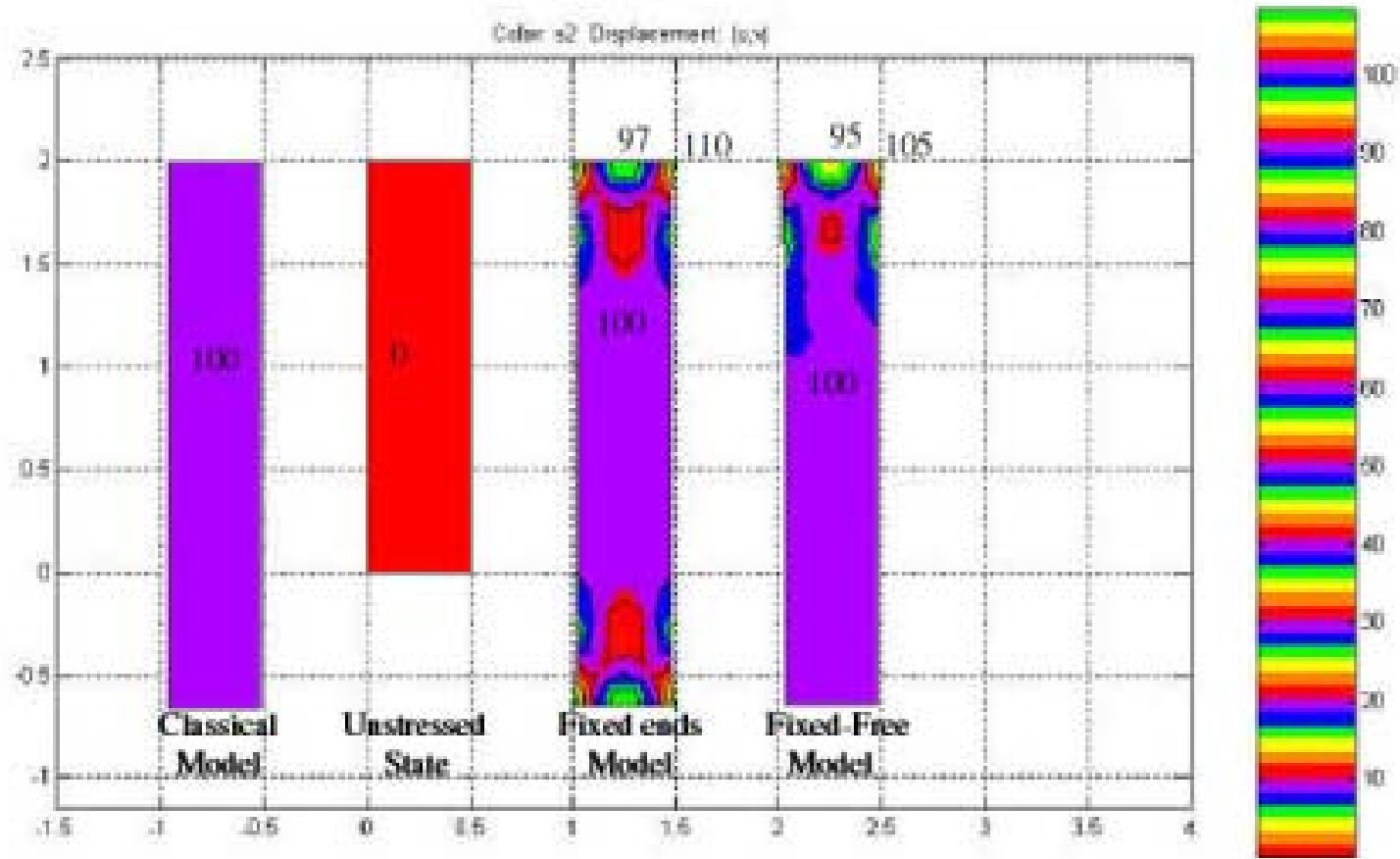

Figure 6-5. Second principal stress of a beam in tension

The final figure, Figure 6-6, is not from the same series of models presented. A variation in boundary conditions produced a deformed shape similar to the previous models, but a field of a different pattern. The top end is fixed with Dirichlet condition, and the bottom is a free surface (Neuman), but the sides have an applied traction downwards in the negative ydirection (Neuman). The previous models have left the side surfaces free. 
This is not a typical boundary condition but compare with Figure 6-2 to see how the field is warped. From the deformed state, this appears as a beam in tension, but the field displays a different shape. This potential field form may be the result of extruding a material or viewing the stress through the thickness of a material clamped in the $\mathrm{x}$-direction and stretched in the negative y-direction. This result also demonstrates the importance of choosing possible and correct boundary conditions for the model, and of the value of performing photoelastic experiments for correlation.

\section{X-Displacement in Beam in Tension}

Displacement

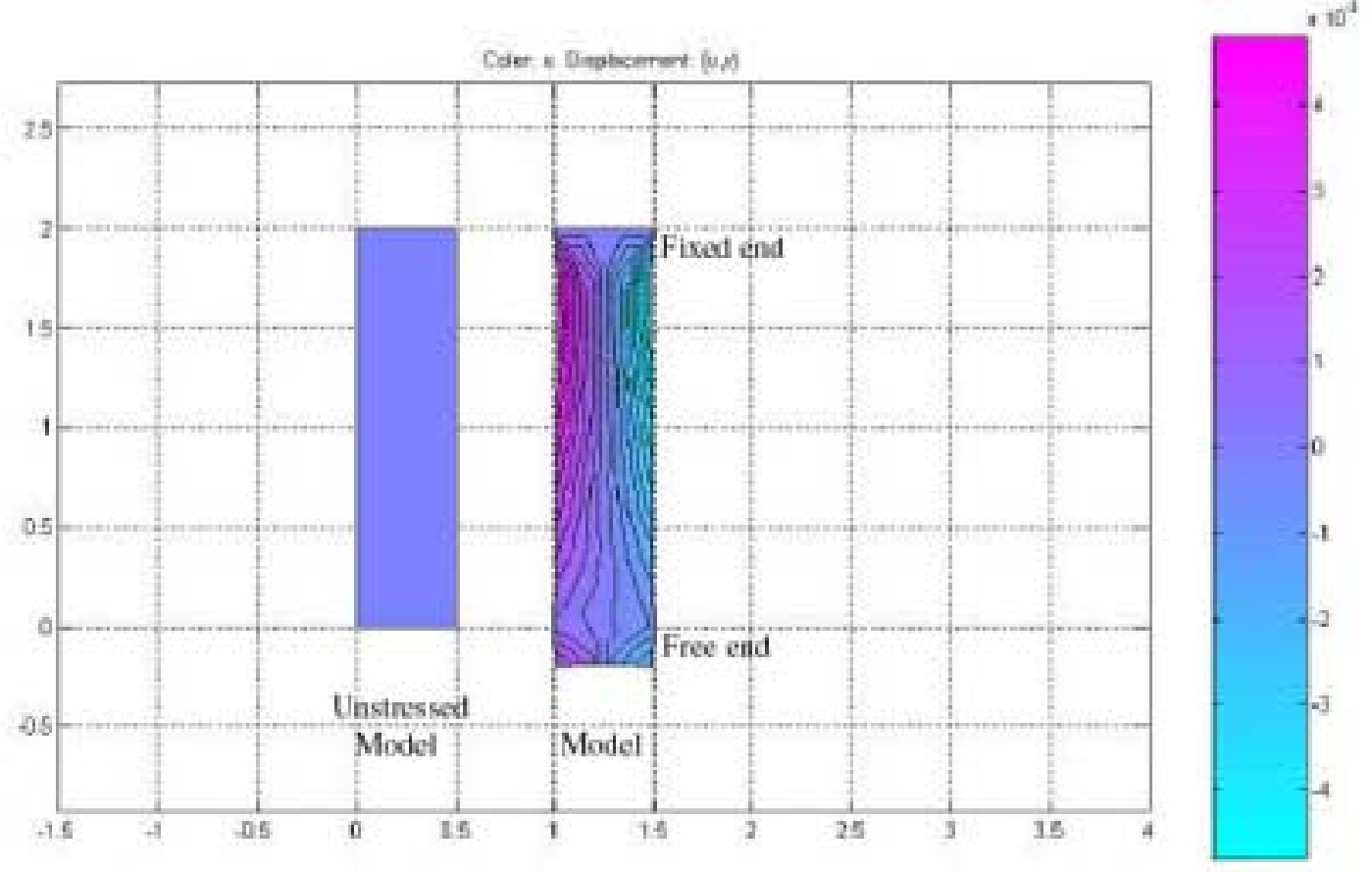

Figure 6-6. X-displacement of altemative boundary conditions for a beam in tension 


\subsection{Analogous Systems}

Potential Force Fields in analogous systems are defined as:

In electrical problems, $\varphi$ represents electric potential or voltage; in magnetic problems, $\varphi$ is the magnetic scalar potential (ampere-turns); in thermal problems, $\varphi$ is the temperature (Celsius); in gravitation, $\varphi$ is the gravitational potential $(\mathrm{J} / \mathrm{Kg})$; in vibration applications, $\varphi$ is the displacement (m); in hydrodynamics and acoustics, $\varphi$ is the velocity potential $\left(\mathrm{m}^{2} / \mathrm{s}\right)$ 90].

\subsection{Fluid and Wave Analogy} $21,36,129,117$

Granular jet experiments [129] produced jets from glass beads (sand) impacted by a lead sphere, similar to jets of water known as Worthington jets, Figure 7-1. This is significant to engineering and geology, demonstrating the similarities in response between granular medium and fluids 129, 134. If attention is confined to plane longitudinal waves moving normal to the faces of a slab, normal shearing forces arise. Then there is quite a good analogy between elastic solids and fluids [117] 


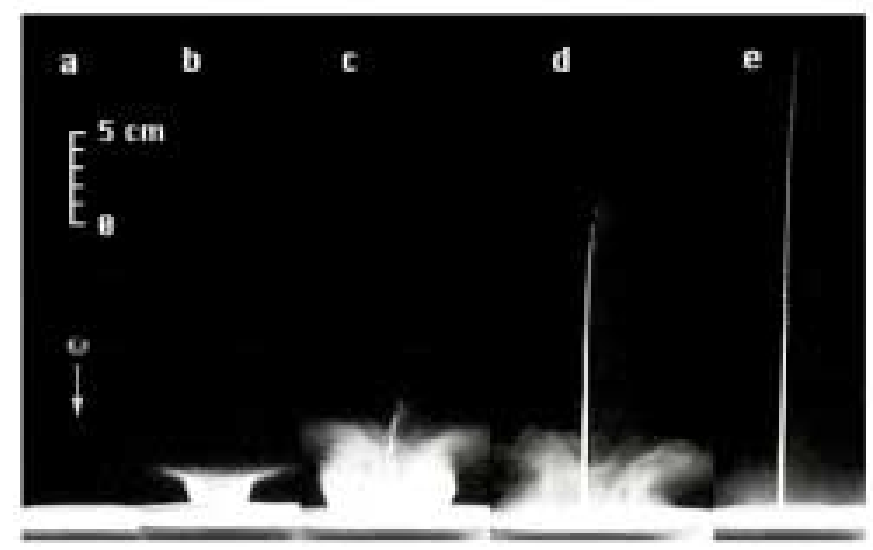

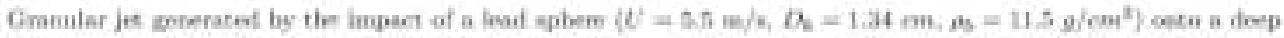

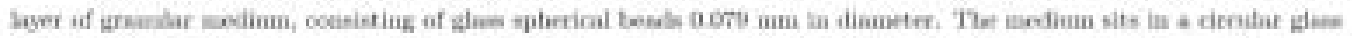

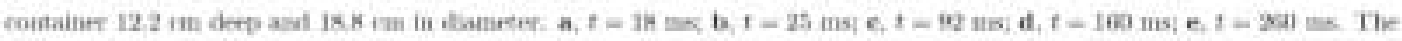

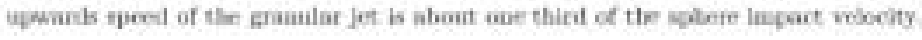

\section{Figure 7-1. Worthington jets produced by spherical beads of sand [129]}

The interaction between grains is very important to microscale mechanics and the strength of materials. Figure 8-1, from Section 8.1.1 Crystal Structure, demonstrates the complex interactions between grains and dislocations, and the experimental results illustrated in Figure 2-18 and Figure 2-19 show that theory is still not consistent with experimental models. Plasticity is one of the most complicated problems for engineers. During plastic deformation, materials that are solids behave like liquids, especially at high strain rates or impacts $30,37,55,53,59,119,130$.

\subsection{Quantum Analogy}

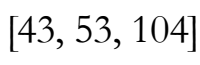

In 1925, Schrödinger described the wave-like nature of particles by equations similar to the differential equations that describe acoustic, elastic, and electromagnetic waves [104, 135]. 
There are no exact analytical solutions for most forms of the Schrödinger's wave equation only numerical approximations, and spherical harmonics are utilized to obtain series solutions 104, 135. Difficulties in solving the equation are similar to the three-body problem of gravitation [See Section 3.5.2 [ules Henri Poincaré], which is also solvable only numerically $35,54,119$.

Together with the Pauli principle and spin, Schrödinger's equation accounts for the differences in hardness between pure metals and pure semiconductors, and it plays an important role in determining the shear stiffness of solids [3]

Electrons follow field equations similar to acoustical vibrations and electromagnetic waves. The equations are given below in Equation 7-1 [33]:

$$
\begin{aligned}
\text { acoustics } \sim & \frac{\partial^{2} u}{\partial x^{2}}=\frac{1}{v_{s}^{2}}\left(\frac{\partial^{2} u}{\partial t^{2}}\right) \\
\text { electromagnetism } \sim & \frac{\partial^{2} \mathrm{E}}{\partial x^{2}}=\frac{1}{c^{2}}\left(\frac{\partial^{2} \mathrm{E}}{\partial t^{2}}\right) \\
\frac{\partial^{2} \mathrm{~B}}{\partial x^{2}} & =\frac{1}{c^{2}}\left(\frac{\partial^{2} \mathrm{~B}}{\partial t^{2}}\right) \\
\text { quantum field } \sim \quad \frac{\partial^{2} \Psi}{\partial x^{2}} & =\left(\frac{p}{E}\right)\left(\frac{\partial^{2} \Psi}{\partial t^{2}}\right)
\end{aligned}
$$

Equation 7-1. Analogous equations of potential fields [53]

In the acoustic equation, the velocity is the speed of sound (of the transmission material); for the electric field (E) and the magnetic field (B), the speed is light-speed, $c$; and for the 
quantum field, the speed is defined as $\frac{E}{p}$ or energy over momentum. The field variable is the probability amplitude $(\Psi)$ for the quantum case, and $\Psi^{2}$ is the probability density 53 . This is analogous to the field variables $\mathrm{u}, \mathrm{E}$, and $\mathrm{B}$, which signify respectively the displacement amplitude, the electric field amplitude, and the magnetic field amplitude [53]. (Also, to find the intensity of the EM fields, the square of the field variable is calculated.)

\subsection{Electromagnetic Analogy} 9. $43,88,119,135$

The electromagnetic analogy is very fitting, because James Clerk Maxwell both derived electromagnetic equations and introduced experimental photoelasticity [75, 84, 88, 135. Electrostatics must also satisfy Laplace's equation when charge densities are zero [135. When there is a total charge density, then Poisson's equation must be satisfied, which sets the Laplacian equal to a constant [135].

Most PDE solvers are equipped to handle electrostatic problems because they are more commonly approached using potential functions. However, any problems with potential fields use the same algorithms to find numerical values and represent fields, including: gravitation, atomic interactions, fluid flow, thermal, electromagnetic, as well as mechanical stress and strain $52,68,78,90,109,131,135$. PDE software is usually designed to handle any of these fields, because it is only a matter of changing the labels of coefficients; the programming remains unchanged for any field solution. 


\subsection{Advances in Computation and Material Science}

"... you know the right equations but they're just not helpful. Y ou add up all the microsopic pieces and you... cannot extend them to the long term. They're not what's important in the problem. It completely changes what it means to Know Something." F eigenbaum [54]

Analytical solutions to the field equations for many general classical mechanics problems have long been unattainable. The approximations to the solution of the field equations, made by Airy [Section 3.4.4], Westergaard [Section 3.4.5], and others, are simplifications obtained by relaxing certain boundary conditions. Advances have been made in mathematically formulating and solving PDE's, in the capacity of computers to numerically solve PDE's, and in sister sciences that use potential fields similar mathematically but of physically different natures. These advances can be applied to mechanical problems with the result of more realistic behavior in models, and closer correlation to experimental results. Computer programs for solving PDE problems are becoming more prominent and widely used.

\subsection{Applied Math and Modeling $23,31,32,40,43,44,45,46,49,53,50,[18,121,124]$}

CMSN, the Computational Materials Science Network, defined one of the greatest challenges in realistic simulation of the mechanical behavior of polycrystalline materials as: the unification of models from different length and time scales [73. Many of the sources and funding agencies recognize that this must be a cross-discipline effort. Multiscale Simulation of Complex Materials, or MUSIC, is a multidisciplinary project being explored by the Advanced Materials Division of ENEA in Rome, Italy (in a white paper by F. Cleri, 
May 1999). Molecular dynamics simulation is using interatomic potentials to model shockinduced plasticity, phase transformations, and dislocation mobility. This work is being carried out at LANL, Brown University, Cal Tech, MIT, LLNL, and in St. Petersburg, Russia. Molecular dynamics (MD) computer simulation combines time-scales to determine viscosity of liquids (similar to shear modulus in solids) with respect to time from ensemble averages of the stress fluctuations, and is described as similar to recent calculations of elastic constants of solids from strain fluctuations with spatial dependence rather than time dependence observed in liquids [60].

Mathematical improvements include:

- New advances to the solutions of elliptic problems, significant to potential theory [62];

- Generating cellular patterns [101, 138];

- FE improvement using potential theory for elliptic PDE solver;

- Fast field solvers for the Poisson equation (Laplacian equal to a constant);

- Successive node reduction used by $\mu$ s-Thermanal (Microsystems on IC chips) and 2D-Sunred [62, 124]; and

- Finite element method of stress extraction using complementary energy principle [141]. 


\subsubsection{Crystal Structure}

42, 62, 70, 73,

Improvements in modeling microstructure include: three-dimensional density functions (or Morse functions) are used to describe the positions and crystal topology of characteristic elements neglecting quantum effects [62] and Ortep-3 used for the stereoscopic modeling of crystals; see figures in [31, 63]. Quantum mechanical effects, like resonant tunneling, superposition, and cellular automata based on quantum dots, are being developed to create quantum computation [112]. The EMSN [73] model utilizes parallel computing and is related to topology [63, 31]. Modeling of grains at the microscale is the hardest and most significant advance towards bridging the scales [112].

New molecular software, Stability Constants (SC-) Database contains the definitive collection of metal-complex stability constants from all significant publications in journals from 1898 to 2000. Presently the database contains 85,000 records from 19,000 references of 8,000 ligands.

Figure 8-1 is the most cross-referenced figure in this thesis. It visually represents many of the issues in modeling microscale behavior. Figure 8-1 is from the paper A tomic-scale Simulations of the Mechanical D eformation of $\mathrm{N}$ anocrystalline $\mathrm{M}$ etals, and contains the response of 16 grains and 100, 000 atoms. Note the discontinuities in strain at the grain boundaries, this is why conservative field based theories do not apply at this scale. 


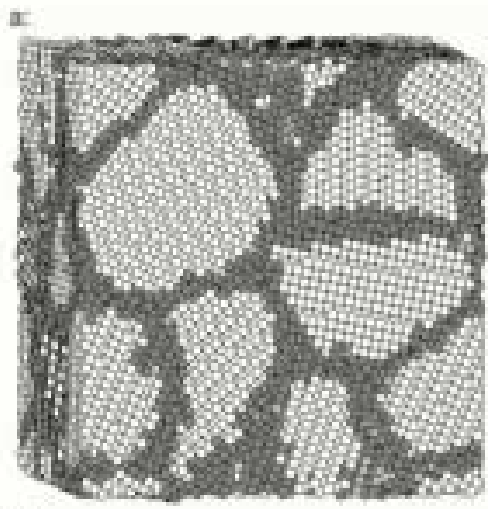

b

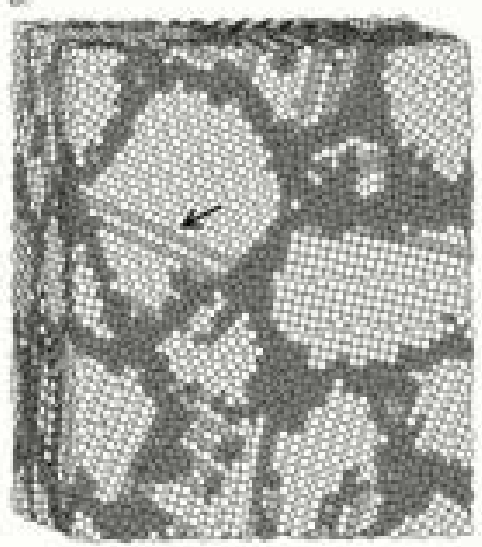

The ititial a) and firal fol cenferunatiun of a nancrys. talline creper syuem deformed $39 \mathrm{~h}$ at $300 \mathrm{~K}$. The sysum contain apreximach 100000 atom amaneet in 16 grains, giving in 2. trate yna dameter of 5.2 um. Alama afe evdor-eaded accondisy 10 the bial crovallime coler. Whise aicess are in a perfect fer emi-

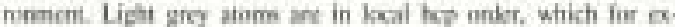
ample comesponds io stackirg tuils. Aocans in any cather envinonInet lin min bounderies and diskestion conest an coliest darh

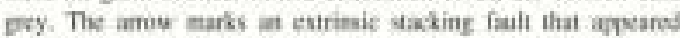
darime the deformation. It was conses thy two Shockdey jurtial moving thungt the grain as adjacent crysal plates.

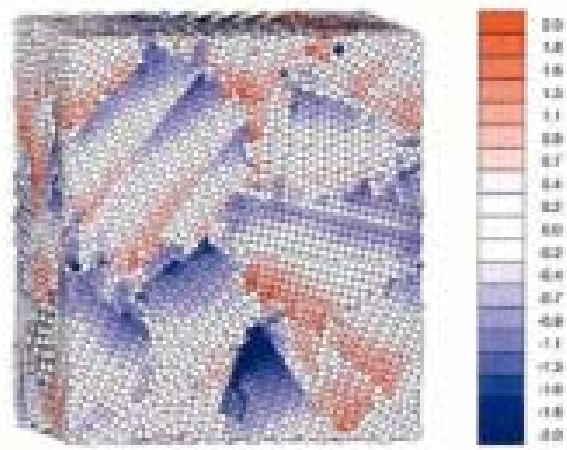

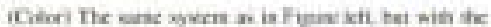

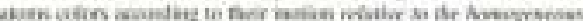

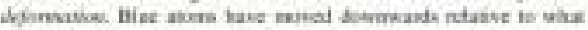

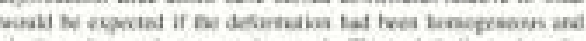

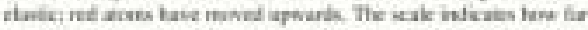

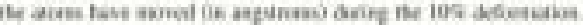

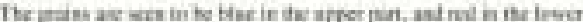

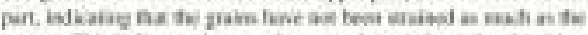

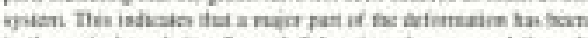

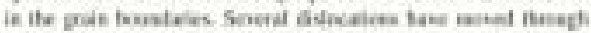

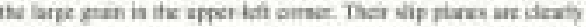
wes
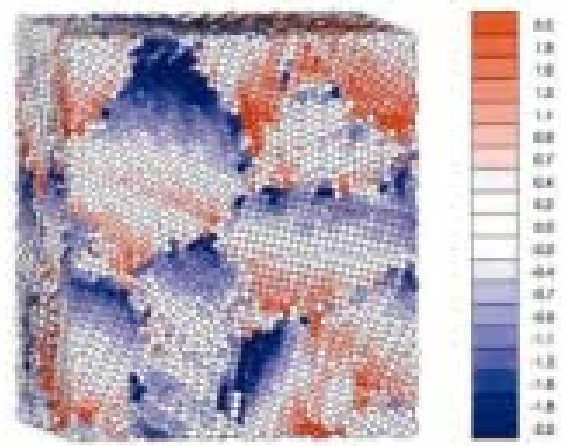

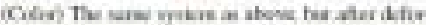

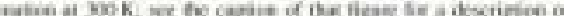

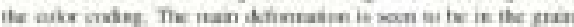

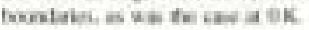

Figure 8-1. Figures from microscale grain simulation. Note: bottom right graph of the rate dependence [118] 


\subsubsection{Modeling Fracture}

20, $21,2,23,110,118,121$

Dynamic cracking analysis, uses ABAQUS and DYNA3D, to model dynamic crack formation and propagation was simulated with starter cracks embedded in the model and more realistic based models utilizing interfacial cohesion forces combined with typical

continuum finite elements [118]. This is important for highly explosive materials [20, spalling, and high deformation manufacturing techniques. Griffith crack and Westergaard stress functions contributed to the success of these models. VIP, a computer program using five mechanical parameters (experimentally defined), produced excellent similarities to photoelastic results of fringes around crack tips, Figure 4-4 [44]. Studies of fracture suggested a new material parameter, incubation time as the minimal interval needed to initiate fracture in high-rate loading conditions, and the only parameter for dynamic strength [1].

\subsubsection{First Principles Calculations and Statistical Mechanics [2, 38, 42,}

Caltech Center for Simulation of Dynamic Response of Materials is using first principles computation to model materials from the quantum level using successively larger scales of time and length to produce parameters useful for continuum calculations [ص. Density functional theory (DFT) is producing excellent correlation to ground state properties, while Quasiparticle theory (QT) is used to adjust the DFT model to account for excited states. As of 1999, molecular dynamic simulations were preformed modeling 1 million particles, at 
distances of $25 \mathrm{~nm}$, for time scales of $10 \mathrm{~ns}$, and using up to 500 processors [112]. 16 grains and 100,000 atoms are modeled in Eigure 8-1, demonstrating stress concentrations at grain boundaries [118]. First principles computation for prediction of material properties is also being conducted at MIT according to the 2002 Material Research newsletter. Molecular dynamics (MD) computer simulation combines time-scales to determine viscosity of liquids from ensemble averages (with respect to time) of the stress fluctuations 600 and is described as similar to recent calculations of elastic constants of solids from strain fluctuations with spatial dependence rather than time dependence observed in liquids. Evolutionary schemes have also been developed for finding new materials with specialized properties [2, 19]. Mathematical models of hysteresis and its application to problems of finding minimum energy configuration were discussed at the Understanding Complex Systems Symposium in Chicago in June of 2001 [ 49

Statistical mechanics has problems being applied to isolated nanostructures 53, 103, because these structures have a significant statistical variation in properties that are not well understood and fluctuate with time [112].

Northwestern University has been studying and modeling high strength steel using quantum theory and supercomputing at the Pittsburgh Supercomputing Center. Greg Olson and Art Freeman began their collaborative efforts in 1985, and in 1991, their work was responsible for the design of a new kind of steel for bearings used in the space shuttle. 
Computational Mechanics using mesh-less boundary node method (BNM) are being used for three-dimensional potential theory, linear elasticity, and fracture mechanics, as well as adaptive meshing. The random walk method is also being explored in an attempt to find solutions to solid and structural mechanics problems.

An efficient method for solving parabolic partial differential equations for quantum mechanical solutions is implemented in Mathematica for solving numerical simulation experiments: a double slit experiment; tunneling (barrier penetration); scattering of a particle from a cylindrical potential barrier; and interaction of two wave-packets. This is an application of solving the two-dimensional time-dependent Schrödinger's equation [See Mathematica website].

Using density functional theory from a chemistry viewpoint, Figure 8-2 is a simulation of the elements in lock and key formation showing the chemistry of how elements fit together to form compounds [See text in figure captions]. This type of software also models complicated compounds like proteins and amino acids, and can be used to determine if reactions will occur [17]. This is very important for bioengineering applications where reactivity is a vital concern. 
Decument Caption-Depictions of atoms and functional groupings of atoms as space-filling objects - regions bounded by the intersection of the interatomic surfaces and the van der Waals envelope of the electron density. The second row hydrides $\mathrm{AH}_{\mathrm{h}}$ where $\mathrm{A}-\mathrm{Li}, \mathrm{Be}, \mathrm{B}, \mathrm{C}, \mathrm{N}, \mathrm{O}$ and F. Note the change in the size and form of the hydrogen atom, from one characteristic of the hydride ion in $\mathrm{LiH}$ to the positively charged one in $\mathrm{HF}$, wherein the atom has been stripped of more than half of its electron density. Bottom Right - The zero value surfaces of 2 for carbon monoxide (left) and borane, BH3(right). These surfaces enclose regions where the electronic charge is maximally concentrated and they define the reactive surface. The same surfaces also show the inner shell charge concentrations on the carbon and boron nuclei, evident as small inner spheres. The molecules are orientated so that the "lump" in the valence shell charge concentration (VSCC) of carbon is aligned with the "bole" in the VSCC of boron. The VSCC of boron is reduced to a belt-like distribution lying in the plane of the hydrogen nuclei, giving the base atom direct access to the core of the boron. the feature that makes $\mathrm{BH} 3$ a strong Lewis acid. Note the torus of charge depletion encircling the carbon nucleus. This feature corresponds to the localization of the * antibonding orbital on carbon.

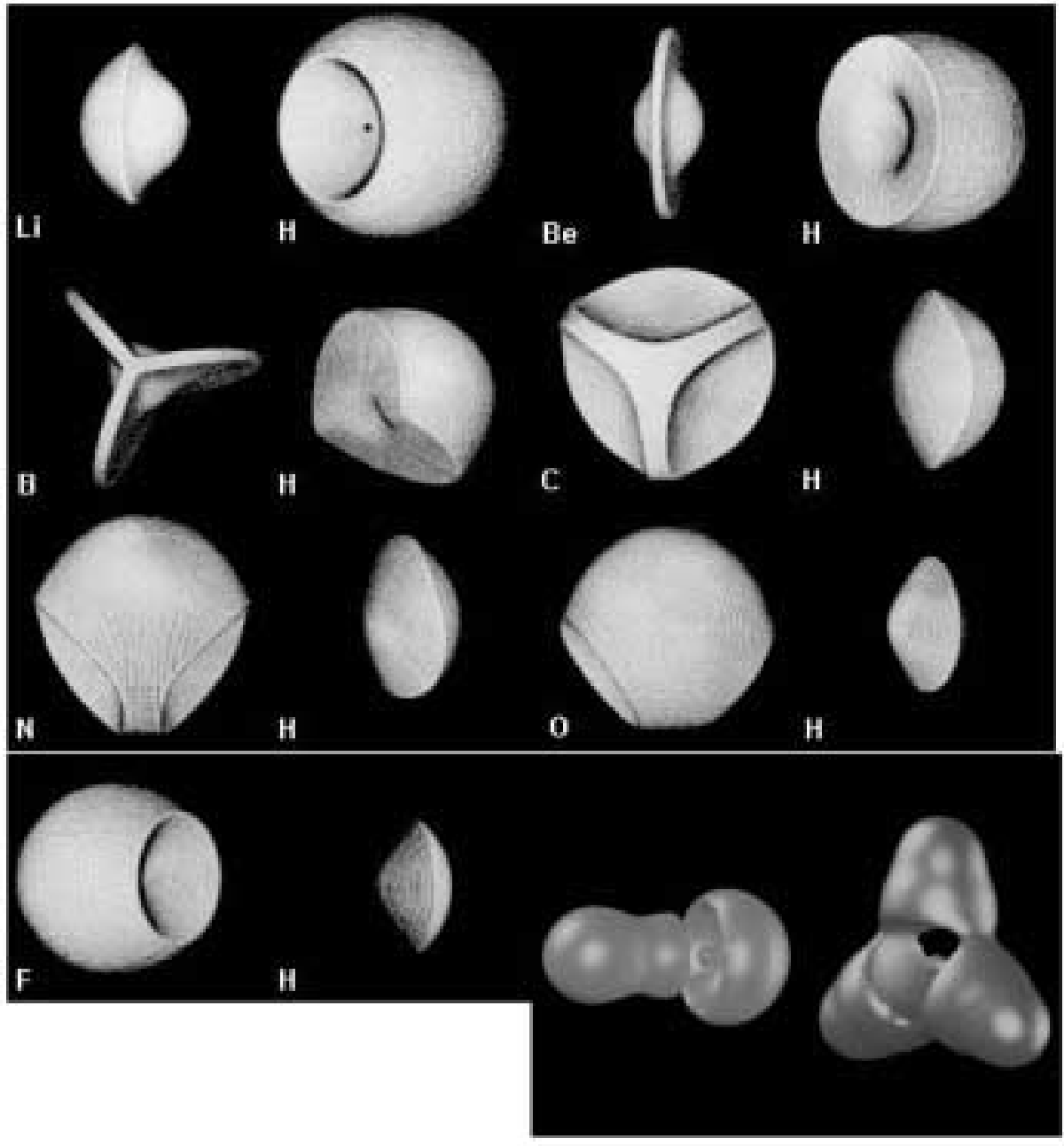

Figure 8-2. Bader's atomic simulations using quantum and functional theory for chemistry diagrams [17] 


\subsection{Material Advances in Nanotechnology}

(4, 5, $1,19,0,23,41,0,0,3,0,1,12,118$

The goals of nanotechnology include understanding size-dependence of properties, fluctuations in properties, properties of nanostructures, and assembly at the nanoscale. Nanoscience is expected to solve many long-standing questions in many scientific fields including physics, material science, and mechanical engineering [112]. Areas of active research and development in nanotechnology include: understanding of properties of nanostructures and components; precision, high out-put, inexpensive nano-manufacturing; and designer nano-materials and nanostructures using computer aided tools [112]. Both kinetic theory and thermodynamics is being studied to understand relative stability of different structural phases and to map phase transformations in nanostructures [12]. Funding agencies are interested in cross-discipline research and collaboration.

Micro and nano-technology are an open frontier including: thin films, micro-testing devices, nano and micro-components, manufacturing methods, and biological applications. Some samples of the wealth of investigative possibilities are the quantum corral, carbon nanotubes gears, and the quartz crystal microbalance. The Quantum corral is constructed of iron atoms arranged with the tip of a tunneling microscope on a copper surface, Figure 8-3. The goal of this component is to trap electrons and force them into particular quantum states (superposition), a component for quantum computing. The carbon nanotubes, Figure 8-4. with benzene teeth is a gear for futuristic NEMS, nanoelectromechanical systems [12] 


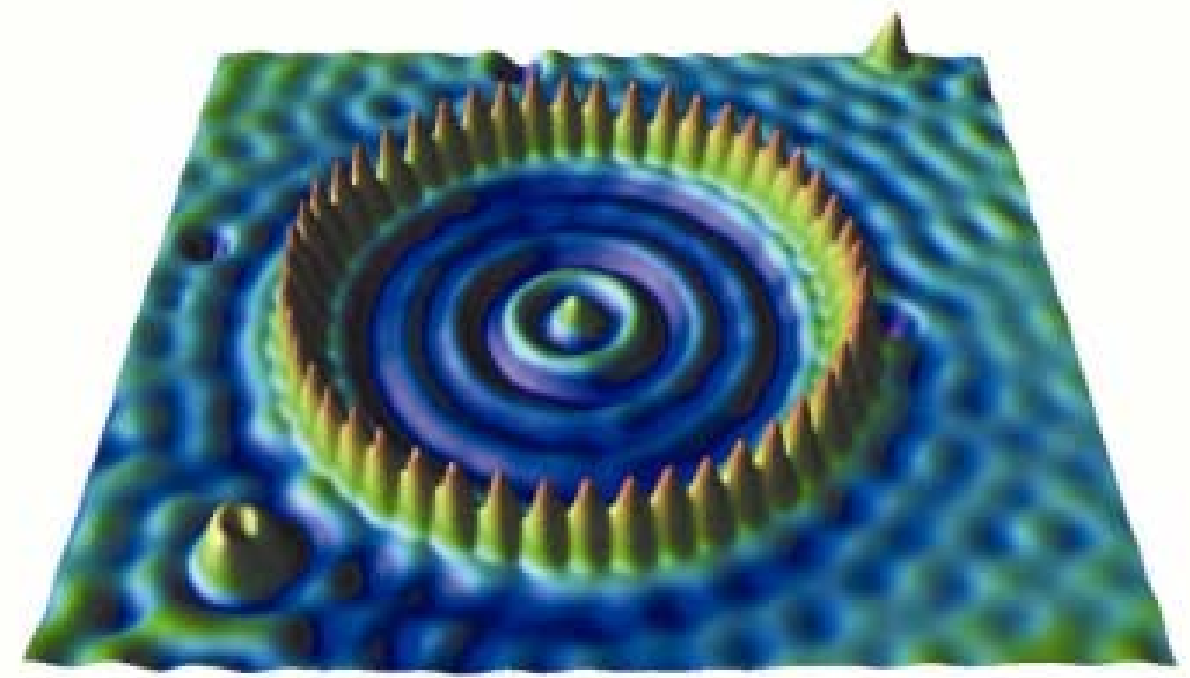

STM image of a qua ntum corral (c ourtesy IBM Research Division).

Figure 8-3. Q uantum corral nanotech component [112]

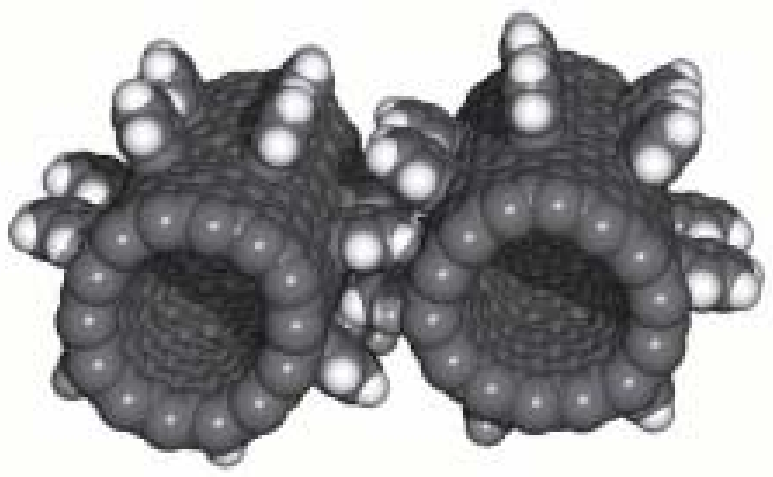

Carbon nanotube-based gears with benzyne teeth (Han et al. 1997; reproduced by permission).

Figure 8-4. Carbon nanotube with benzene teeth gear, nanotech component [112] 
Combining components into machines and analyzing their mechanical systems and stress distributions is particularly suited for mechanical engineers, but the properties of these components must be understood before design can be contemplated.

Quartz crystal microbalance (QCM) is being used at Cambridge to detect the acoustic noise of bonds breaking and has applications to biosensors [11]. QCM is being used to investigate bonds and their relative strengths, and is one of the problems mentioned in Section 1.3 Examples of Specific Nanotech Problems.

Materials references include: $[1,4,5,6,7,15,16,19,26,41,56,59,66,06,98,107,122,123$, $125,128,130$ 


\subsection{In Conclusion}

The ability to design at the nano-level, to choose and create new materials or applications and to model from atomic (first principles) up to the bulk level is beyond present engineering ability. It requires a cross discipline investigation and synthesis of methods and ideas from different fields. The mathematical foundation of this work comes under potential field theory and covers most of the goals of engineering at the nano-scale. Recent computer advances make this approach realistic and numerically solvable.

Potential field theory has been used by physicists to solve static electromagnetic field problems since Maxwell derived the equations and is the recognized exact solution to mechanical stress and strain fields. In the past, considerable work has been done to analytically solve these very complicated partial differential equations, but recent computer advances were necessary to obtain numerical solutions. The nature of the field depends on scale, but not the solution. Failure criteria are dependent on strength and although the idea of a material performing at a percentage of its maximum, or theoretical strength, is not important for bulk calculations, it has become important to nano-scale calculations and especially in modeling material response. The constants and variables of strength are not scale invariant; these material properties depend on scale and cannot be neglected at scales smaller than $1 \mathrm{~mm}$.

Because Potential theory is so flexible and is used to model atomic interactions, grain interactions, defects, and cracks, making the natural extension to bulk mechanics using PDE solvers sets the stage for combining the levels into a truly multiscale application. 
To circumvent the need for costly, explorative experiments at the nano-scale, theoretical strength models can be used for assigning values to the measures of strength and the crystallographic constants, and experimentation can be used to verify the computer models.

The upper bound of material strength is close to the strength achieved by nanocomponents, as observed from the few experiments that have already been conducted [See 23. 66, 38, 23, 112]. This gives engineers the opportunity to design nano-mechanical systems making use of the new nano-components. Because of the additional problems of plasticity, dislocations and grains, the micro-scale is still just a modeling tool that needs more work before it can be used as a design tool.

This investigation has also highlighted how important cross-discipline work is to the development and solution of multiscale problems. Each of the variables that contribute to strength must be included in a comprehensive model, not only to demonstrate their effect, but also to observe the limiting conditions (where the variables can safely be eliminated). Some excellent modeling programs have been developed exclusively for the atomic and molecular scale for chemists and physicists. These can serve as the first level to modeling independent of scale and an aid to engineers working and designing with nanocomponents.

\subsection{Limitations}

Potential fields are observed and modeled at every scale. The math is scale invariant, but the material properties vary with spatial and temporal scales. To design at scales other than the 
bulk level requires cross-collaboration to obtain good material models and accurate descriptions of the various fields involved in a particular problem. Setting boundary conditions is always an important issue, as is apparent in Section 6.0 .

Many problems cannot be analytically solved. For inseparable partial differential equations, there are only numerical solutions. Solutions and computer programs utilizing truly threedimensional coordinates that are not spherical are rare. Microscale and plasticity are still difficult to model, and may not be completely defined using potential field theory. Chaos and nonlinear processes are not adequately understood at this scale, and although strides have been made in microscale modeling, there are numerous conflicts that need to be resolved before microscale modeling becomes an effective design tool.

\subsection{Recommendations}

The next step is using potential theory methods to solve, catalog, and explore the limitations of the method as applied to general and specialized engineering problems. The many problems that can be analytically solved have not been explored and combined for easy reference. This can be expanded into three-dimensional modeling programs and even further by developing four-dimensional models of vibration and impact with time as the fourth variable.

Potential field theory can be applied to all levels and scales in material response simulation; at every level, there is a field controlling the material's response. At the nano-scale, the field

is electromagnetic potential [See Section 7.2 Quantum Analogy and Section 7.3 
Electromagnetic Analogy]; at bulk scale, the potential field can be modeled as stress (or strain); at the micro-scale, a potential field, made of elastic waves, drives dislocations and grain boundary interactions, requiring a more sophisticated model.

The main reason for adopting potential field theory as opposed to a more familiar finite element solver that uses traditional bulk mechanical solutions, like Ansys, Abaqus, and others, is that potential theory can be applied through all the size and time scales. The algorithms and methods for numerically solving quantum and microscale problems are equally applicable to solving the PDE's governing the bulk problems. The extension from atomic behavior to grains and to bulk structures can be accomplished using potential theory and massive computational resources. 


\section{BIBLIOGRAPHY}

1. Brittle and Plastic Failure: Phys1250 L ecture 11.

http://newwt.phys.unsw.edu.au/ epe/1250.L11/1250.L11.small.html 2000

2. Center for Simulation of Dynamic Response of Materials: First Prinaples

Computation of Materials Properties. http://www.wag.caltech.edu/asci/

Caltech. CA. May 17, 1999

3. Surface Forces: Physical Surface Chemistry.

http://www.cs.uwindsor.ca/www/gully/90-53/StudentPapers/surfaceforces.pdf 2000

4. Buckytubes - Properties. http://www.azom.com/details.asp?articleID=1294

Carbon Nanotechnologies, Inc.. Texas. February 19, 2002

5. Nanomechanics of Defects in Solids.

http://www.cacr.caltech.edu/ASAP/news/specialevents/site.visit00/presentations /olmsted.pdf Caltech. 1999

6. Scientific Principles.

http://matse1.mse.uiuc.edu/ tw/ceramics/prin.html

Materials Science and Technolology. 2000

7. Plastic Deformation in Polycrystalline Materials.

http:// faculty.eng.fui.edu/ $\sim_{\text {wu/trpchp7.doc }} 000$

8. Shock C ompression of C ondensed Matter Meeting 2001 Scientific Program.

http://www.eps.org/aps/meet/SHOCK01/baps/abs/S280.html

Molecular Dynamics, Plasticity, and Phase. Bulletin of the American Physical Society. Vol 46, No 4. European Physical Society. Atlanta, Georgia. June 24-29, 2001

9. Electromagnetics: Introductory Concepts.

http://bbs1.nju.edu.cn/ file/chap01.pdf CRC Press. 2001

10. Laplace-Y oung and Bashforth-A dams Equations.

http://www.firsttenangstroms.com First Ten Angstroms.

Portsmouth, Virginia. November 24, 2000 
11. Adam, David. Bond-breaking Bangs. Nature. Macmillan Magazines Ltd. June 6, 2000

12. Airy, George Biddell. $\mathbf{O}$ n the Strains in the Interior of Beams.

http://www.jstor.org

Philosophical Transactions of the Royal Society of London. Vol 153.

The Royal Society. London. 1863

13. Albers, Vernon M. Suggested Experiments for Laboratory Courses in Acoustics and Vibrations. ISBN-0271011041. Acoustical Society of America.

The Pennsylvannia Press. University Park, PA. 1972

14. Alessandrini, G and and M Sigalotti. Geometric Properties of Solutions to the Anisotropic p-Laplace Equation in Dimension Two.

http://www.emis.de/journals/AASF/Vol26/alessand.pdf

Annales Academiæ Scientiarum Fennicæ Mathematica. Vol 21, 249-266.

Academia Scientiarum Fennica. Trieste, Italy. 2001

15. Amateau, Maurice F. 1. Introduction and Definitions: and 2. Theoretical Properties of

Materials. http://www4.esm.psu.edu/academics/courses/emch471/

Penn State. University Park, PA. 2003

16. Argon, Ali S. Physics of Strength and Plasticity. ISBN-0262010305.

The MIT Press. Cambridge, MA. 1969

17. Bader, Richard F W. Theory of Atoms in Molecules.

http://www.chemistry.mcmaster.ca/faculty/bader/aim/aim 0.html

McMaster University. Ontario. 1995

18. Baker, G L and J P Gollub, and J A Blackburn. Inverting Chaos: Extracting system parameters from experimental data.

Chaos. Vol 6, No.4. American Institute of Physics. November 7, 1996

19. Ball, Philip. Metallurgists blend by numbers: Computers speed the search for new engineering materials. http://www.nature.com/nsu/020617/020617-13.html

Nature. Nature Publishing Group. June 24, 2002

20. Banerjee, Biswajit. Micromechanics-Based Prediction of Thermoelastic Properties of High Energy Materials: D issertation.

University of Utah. January 2002 
21. Barannyk, L F and and B Kloskowska, VV Mityushev. Invariant Solutions of the Multidimensional Boussinesq Equation.

http://books.hindawi.com/9775945038/

Second International Conference Symmetry in Nonlinear Mathematical Physics, Kyiv, Ukraine. Symmetry in Nonlinear Mathematical Physics. Vol 1, 62-69.

Hindawi Publishing Corporation. Stupsk, Poland. 1997

22. Barannyk, L F and P.Sulewski. Exact Solutions of the Nonlinear Diffusion Equation. Symmetry in Nonlinear Mathematical Physics. V.2, 429-436. Stupsk, Poland. 1997

23. Baskes, Mike I and M F Horstemeyer, S Plimpton, D Hughes, D Plattillo, B Somerdat. Examination of Length Scale Effects on Plasticity in Metals by Use of A tomistic Simulations.

http://www.msd.anl.gov/groups/im/cmsn/BaskesPresentation/sld001.htm

Microstructural Effects on the Mechanics of Materials Workshop. Argonne National Laboratory. Argonne, IL. June 5-7, 2000

24. Batra, R C and F Dell'Isola, and S Vidoli. A Second-0 rder Solution of SaintV enant's Problem: for a Piezoelectric Circular bar U sing Signorini's Perturbation M ethod. Journal of Elasticity. Vol 52. Kluwer Academic Publishers. 1998

25. Beals, M and L. Gross, and S. Harrell. Spider Silk: Stress-Strain arres and Y oung's Modulus. http://www.tiem.utk.edu/ mbeals/spider.html TIEM. Tennessee. 1999

26. Beaucage, Gregory. Deformation Morphologies and Toughening of Polymer Systems: Ch. 3.

http://www.eng.uc.edu/ gbeaucag/Classes/Morphology/Chapter3html/Chapter3 html University of Cincinnati. Cincinnati, OH. April 27, 1998

27. Benaroya, Haym. Mechanical Vibrations: A nalysis, U noertainties, and Control. ISBN013948373x. Prentice Hall. Upper Saddle River, NJ. 1998

28. Borchardt-Ott, Walter. Crystallography. $2^{\text {nd }} E$ dition. ISBN-3540594787. Springer-Verlag. Berlin. 1995

29. Bozhevolnaya, Elena and Arne Kildegaard. Numerical Treatment of the Photoelasticity Data. http://www.ime.auc.dk/People/employees/eb/Notes/photoelasticity.pdf Institute of Mechanical Engineering. Denmark. August 2000 
30. Budynas, Richard G. Advanced Strength and Applied Stress Analysis. ISBN007008985x. McGraw-Hill. Boston. 1999

31. Burnett, Michael N and and Carroll K. Johnson. ORTEP-III Computer Program: 0 ak Ridge Thermal E llipsoid Plot Program for Crystal Structure Illustrations. http://www.ornl.gov/ortep/ortep.html Oak Ridge National Laboratory. Oak Ridge, TN. 2001

32. Callister, Jr, William D. Materials Science and Engineering: Fifth E dition. John Wiley \& Sons, Inc. New York, NY. 2000

33. Casati, Giulio. Q uantum Chaos. http://www.aip.org/journals/chaos/391 1.pdf Chaos. Vol 6 (3). American Institute of Physics. June 1996

34. Clark, Al. Contours for Laplace Equation.

http://www.me.rochester.edu/courses/ME201/webexamp/lapcon.pdf Rochester, NY. June 20, 2002

35. Cohl, Howard and J.E. Tohline, A.R.P. Rau, and H M Srivastava. Developments in determining the gravitational potential using toroidal functions. http://hcohl.shell42.com/an/Poster form at 200th IAU symposium on binary star formation (Potsdam, Germany April 10-15, 2000). Astron.Nachr. Vol 321 5/6,363 372. October 5, 2000

36. Courant, Richard and Kurt Otto Friedrichs. Supersonic Flow and Shock Waves: A pplied M athematical Scienos 21. ISBN-0387902325. Vol 21. Springer. New York. 1999 (1948 original)

37. Dally, James $\mathrm{W}$ and William $\mathrm{F}$ Riley. Experimental Stress Analysis. ISBN0070152047. McGraw-Hill. New York. 1978

38. Donovan, B and J F Angress. Lattice Vibrations. ISBN-41610410 X. Chapman and Hall LTD. London. 1971

39. Douglas, J F and E J Garboczi. Intrinsic Viscosity and the Polarizability of Particles Having a Wide Range of Shapes. http://ciks.cbt.nist.gov/ ' garbocz/paper58/paper58.html Advances in Chemical Physics 91. Gaithersburg, MD. 1995 
40. Edwards, S F and D V Grinev. The Tensorial Formulation of V olume Function for Packings of Particles.

http://www.poco.phy.cam.ac.uk/ dg218/ces.pdf

Chemical Engineering Science. Vol 56 (19). Pergamon. UK. January 1, 2002

41. Eichhorn, S J and and R J Young. The Y oung's Modulus of a Microcrystalline Cellulose.

http://www2.umist.ac.uk/material/research/raman/webpage/Raman pubs/2001 15.pdf Cellulose. Vol 8, 197-207. Kluwer Academic Publishers. Netherlands. 2001

42. Farge, $\mathrm{Y}$ and M P Fontana. Electronic and Vibrational Properties of Point Defects in Ionic Crystals: Series: D efects in Crystalline Solids. ISBN-044852727.

North-Holland. Amsterdam. 1979

43. Farina, C and J J Passos, and A C Tort. Q uantum Maxwell Stresses and the Casmir Effect: $M$ ax well Stress T ensor and the E ledromagnetic Field C orrelators. http://www.sbf1.if.usp.br/eventos/enfpc/xxi/procs/res181/res181.html http://www.sbf1.if.usp.br/eventos/enfpc/xxi/procs/res181/node2.html Encontro Nacional de Fisica de Particulas e Campos. Brazil. December 22, 2000

44. Ferber, F and O Hinz, and K P Herrmann. VIP: A Computer Program for the $D$ etermination of Fracture Mechanical Parameters. 10th International Conference on Experimental Mechanics. Vol 1, 217-222. Lissabon, Portugal. July 18-22, 1994

45. Field, Jr, R V and J R Red-Horse, and T L Paez. A Nondeterministic Shock and Vibration A pplication U sing Polynomial C haos Expansions. http://www.usc.edu/dept/civil eng/johnsone/pmc2000/sessions/papers/p087.p $\underline{\overrightarrow{\mathrm{df}}}$

8th ASCE Specialty Conference on Probalistic Mechanics and Structural Reliability. American Society of Civil Engineers. South Bend, Indiana. 2000

46. Flanagan, Gerry. An Efficient Stress Function Approximation for Free-Edge Stresses in Laminates. http://www.materials-sciences.com/papers/EdgeTheoretical.pdf Materials Sciences Corporation. Fort Washington, PA. January 4, 2002

47. Forest, $\mathrm{S}$ and and R. Sievert. Elastoviscoplastic Constitutive Frameworks for Generalized Continua. http://www.mat.ensmp.fr/Personnel/Forest/publi/Forest Sievert ActaMech03.p $\underline{\mathrm{df}}$

Acta Mechanica. Vol 160, 71-111. Springer-Verlag. Austria. January 16, 2003 
48. Fraser, Hamish L. D efects and Strengthening Mechanisms.

http://mse-p012.eng.ohio-state.edu/ fraser/mse565/Strength mechs pp91-

105.pdf MSE 565 - Crystal Mechanics. Ohio State University. 2002

49. Friedman, Gary. Hysteresis and Return-Point Memory in Complex Systems. http://www.how-why.com/ucs/abstracts.html\#friedman

Understanding Complex Systems Symposium. Chicago, IL. May 15, 2001

50. Gao, H and Y Huang and W D Nix. Modeling Plasiticity at the Micrometer Scale. Naturwissenschaften. Vol 86. Springer-Verlag. 1999

51. Gerstner, Ed. Second Law Broken: Small-Scale E nergy Fluctuations Could Limit Minaturization. http://www.nature.com/nsu/nsu pf/020722/020722-2.html

Nature. Nature Publishing Group. July 23, 2002

52. Gilliam, Dave and Lawrence Schovanec. C hapter 6. Partial Differential

Equations http://texas.math.ttu.edu/ gilliam/ttu/ode pde pdf/Ch6.pdf

Texas Tech University. Texas. 1999

53. Gilman, John J. Electronic Basis of the Strength of Materials. ISBN-0521620058. Cambridge University Press. Cambridge, UK. 2003

54. Gleick, James. Chaos: Making A N ew Scieno. ISBN-0140092501.

Penguin Books. New York. 1987

55. Gregory, R D and and F Y M Wan. On Plate Theories and Saint-Venant's Principle. http://www.elsevier.nl/inca/publications/store/2/9/7/ International Journal of Solids and Structures. Vol 21, No 10, 1005-10024.

Pergamon Press, Ltd. Great Britain. 1985

56. Halem, Henry. Does Glass Flow?: The "G lass Flow" Myth. http://www.glassnotes.com/WindowPanes.html Franklin Mills Press. Ohio. April 1999

57. Hamilton, William Rowan and Edited by David R Wilkins, 2000. On the Solution of the Equation of Laplace's Function.

http://www.emis.de/classics/Hamilton/SolLapl.pdf

Proceedings of the Royal Irish Academy. Vol 6.

The European Mathematical Information Service. 1858 
58. Hamilton, William Rowan and Edited by David R Wilkins, 2000. On the Integrations of $\mathrm{C}$ ertain Equations.

http://www.emis.de/classics/Hamilton/IntEq.pdf

Proceedings of the Royal Irish Academy. Vol 6, 62-63.

The European Mathematical Information Service. 1858

59. Hartog, J P Den. Strength of Materials ISBN-0486607550.

Dover Publications, Inc. New York. 1949, 1977

60. Hess, Siegfried and Martin Kröger, and Denis J. Evans. C rossover Between Shortand Long-time Behavior of Stress Fluctuations and Viscoelasticty of Liquids. http://oips.aip.org/dbt/dbt.jsp?KEY=PLEEE8\&Volume=67\&Issue $=4$

Physics Review E. Vol 67, 4. The American Physical Society. Berlin, Germany. 2003

61. Hinders, Mark. Hand-Held Polariscope: Photoelastic N D E Revival.

http://www.as.wm.edu/Faculty/Hinders/Photo.pdf

The College of William \& Mary. Williamsburg, VA. November 13, 2000

62. Huang, Jingfang and Leslie Greengard. A Fast Direct Solver for Elliptic Partial Differential Equaitons on A daptively Refined Meshes.

http://www.siam.org/journals/sisc/21-4/34623.html Biam Journal of Scientific Computing. Vol 21, No 4. Society for Industrial and Applied Mathematics. New York, NY. 2000

63. Johnson, Carroll $\mathrm{K}$ and Michael $\mathrm{N}$ Burnett. Crystallographic Topology 101: Introduction to Critical N ets. http://www.ornl.gov/ortep/topology.html

Oak Ridge National Laboratory. 1999

64. Kanth, Parmjit Singh. Research in Automated Photoelasticity: Photolastiaty Basics. http://www.mie.utoronto.ca/labs/emdl/people/kanth/photo2.htm University of Toronto. 1997

65. Kelly, A and G W Groves. Crystallography and Crystal Defects. ISBN0471720445. Longman Group Ltd. London. 1970

66. Kelly, A. Strong Solids. ISBN-019851350. Oxford University Press. Oxford. 1973

67. Kittel, Charles. Introduction to Solid State Physics: Seventh E dition. John Wiley \& Sons, Inc. New York, NY. 1996

68. Korner, T W. Complex Methods C ourse P3. 
http://www.dpmms.cam.ac.uk/ twk/CM.pdf

University of Cambridge. UK. December 11, 2002

69. Krause, Carolyn. O rder $\mathbf{O}$ ut of C haos: N eutrons Make Sense of D isordered Materials. http://www.oakridger.com/stories/082798/sns golf.html

The Oak Ridger Online. August 26, 1998

70. Kurzydlowski, Krzysztof Jan and Brian Ralph. The Q uantitative Desciption of the Microstructure of Materials. CRC Press. New York. 1995

71. Lau, C Y and S H Teoh. 3-D Photoelastic Stress A nalysis of the Femur.

http://asme.pinetec.com/bio1999/data/pdfs/a0066834.pdf

Bioengineering Conference. ASME. Big Sky, Montana. June 16-20, 1999

72. LeMaster, Robert A. Stress C oncentration Factors and Notch Sensitivity. http://www.utm.edu/departments/engin/lemaster/Machine $\% 20$ Design/Lecture $\%$ 2004.pdf University of Tennessee at Martin. Tennessee.

73. LeSar, Richard and Dieter Wolf. Microstructural Effects on the Mechanics of Materials. http://www.msd.anl.gov/groups/im/cmsn/cmsn.html une 5-7, 2000

74. Liapunov, A M. Stabilty of Motion. Mathematics in Science and Engineering. Vol 30. Academic Press. New York. 1966

75. Love, A E H. A Treatise on the Mathematical Theory of Elasticity. ISBN0486601749. Dover. New York. 1944, 1927

76. L'vov, Victor and Itamar Procaccia. The Universal Scaling Exponents of A nisotropy in Turbulence and their Measurement.

http://lvov.weizmann.ac.il/Papers Online/129 PhysFluids Anis-Exp\&Exp.pdf Physics of Fluids. Vol 8 (10). American Institue of Physics. October 1996

77. MacFarlane, D L. Numerical Solution to Laplace's Equation. http://www.utdallas.edu/ dlm/graduate emag/Computer Project, C shaped aperture.htm Dallas, Texas. 2/20/03

78. MacMillan, William Duncan. The Theory of the Potential: Theoretical Mechanics. ISBN-0486604861. Dover. New York. 1930, 1958

79. Mandelbrot, Benoit B. Multifractals and 1/ f Noise: Wild Self-A finity in Physics. ISBN-0387985395. Springer. New York. 1999 
80. Mandelbrot, Benoit B. The Fractal Geometry of Nature. ISBN-0716711869.

W. H. Freeman and Company. New York. 1983

81. Martel, Stephen. Westergaard Complex Stress Functions (16).

http://www.soest.hawaii.edu/martel/Courses/GG711/GG711c Lec 16.pdf

University of Hawaii. HW. January 21, 2003

82. Martell, Stephan. Stress Functions in Rectangular C oordinates.

http://www.soest.hawaii.edu/martel/Courses/GG711/GG711c Lec 09.pdf

University of Hawaii. HW. January 20, 2003

83. Maxwell, James Clerk and Edited by Elizabeth Garber, Stephen Brush, and C W F Everitt. Maxwell on Heat and Statistical Mechanics: On "A voiding A ll Personal E nquiries" of M olecules. ISBN-0934223343. Vol 3.

Lehigh University Press. Bethlehem, PA. 1995

84. Maxwell, James Clerk. A Treatise on Electricity \& Magnetism. ISBN0486606368.

Vol 1. Dover. New York. 1954

85. Maxwell, James Clerk. Matter and Motion. ISBN-0486668959.

Dover. New York. 1991 reissue

86. Maxwell, James Clerk. O n Reciprocal Figures and Diagrams of Forces.

The London, Edinburgh and Dublin Philosophical Magazine.

Ser 4, Vol 27, 250-261. Taylor \& Francis. London. 1864

87. Maxwell, James Clerk. Theory of Heat. ISBN-0486417352.

Dover. Mineola, New York. 9th ed. 2001 (original 1888)

88. Meyers, H P. Introductory Solid State Physics. ISBN-0748406603.

Taylor and Francis Ltd. London. 1997

89. Minkel, J R. C harles Lieber: A Big Player in the W orld of the Super-small T urns G old into $\mathrm{N}$ anowire. Popular Science. November 2002

90. Moon, Parry Hiriam and Domina Eberle Spencer. Field Theory Handbook: Induding C oordinate Systems D ifferential E quations and Their Solutions. ISBN-0387027327. Springer-Verlag. Berlin Germany. 1961 
91. Morozov, N F and Y V Petrov. The Incubation Time -: Theory and E x periment. http://www.ts.mah.se/forskn/mumat/activities/conferences/iutam/Morozov.pdf Field Analyses for Determination of Material Parameters Experimental and Numerical Aspects. IUTAM SYMPOSIUM. St. Petersburg, Russia. July 31 - August 4, 2000

92. Müller, W H. On the Interaction Between Cracks and Inclusions. http://www.hw.ac.uk/mecWWW/research/whm/research.htm

Proceedings of the GAMM-Meeting 1994. 75. ZAMM. 1995

93. Nabarro, F R N and A S Argon. Egon O rowan. http://www.nap.edu Biographical Memoirs. V.70, p 260-318. National Academy Press. 1996

94. Novozhilov, V V. Foundations of the Nonlinear Theory of Elasticity. ISBN0486406849. Dover Publications. New York. 1953,1999

95. Ogden, R W. Non-Linear Elastic Deformations. ISBN-0486696480.

Dover. Mineola, New York. 1984

96. Ohnami, Masateru. Plasticity and High Temperature Strength of Materials: Combined Micro- and M accro- M echanical A pproaches. ISBN-1851661190.

Elsevier Applied Science. New York. 1988

97. Olver, Frank W J. Airy and Related Functions: Chapter A I:. http://dlmf.nist.gov/Contents/AI/NIST. College Park, MD. 1998

98. Orowan, E. Surface Energy and Surface Tension in Solids and Liquids. http://www.jstor.org

Series A, Mathematical and Physical Sciences. Proceedings of the Royal Society of London. Vol 316, Issue 1527, p 437-491. The Royal Society. 1970

99. Owen, Mark Philip. Topics in the Spectral Theory of Fourth 0 rder Elliptic

Differential 0 perators. Ph. D . D issertation

http://www.ma.hw.ac.uk/ $\sim$ mowen/research/thesis/thesis.pdf

King's College London. London, England. November 1996

100. Pacey, M N and E A Patterson, and M N James. A Photoelastic Technique for Characterising Fatigue C rack Closure and the Effective Stress Intensity Factor. http://www.immt.pwr.wroc.pl/konferencje/lsmp01/1sm2001_13.pdf 
VII Summer School of Fracture Mechanic, Current Research in Fatigue and Fracture, ZESZYTY NAUKOWE POLITECHNIKI OPOLSKIEJ Seria: Mechanika. Nr kol. 269/2001. Pokrzywna (Poland). 18-22 Jun. 2001

101. Palacios, Antonio and Gemunu H Gunaratne, Micheal Gorman, and Kay A Robbins. C ellular Pattern Formation in Circular Domains.

http://www.aip.org/journals/chaos/463.pdf

Chaos. Vol 7 (3). American Institue of Physics. 1997

102. Parker, Ann. Predicting Material Behavior from the A tomic Level Up.

http://www.llnl.gov/str/Moriarty.html Science \& Technology Review.

LLNL. June 1999

103. Pathria, R K. Statistical Mechanics. ISBN-0750624698.

Butterworth-Heinemann. Oxford. 1972, 1996

104. Pauli, Wolfgang. Wave Mechanics: Pauli L ectures on Physics. ISBN-0486414620.

Vol 5. Dover. Mineola, New York. 1973

105. Perko, Lawrence. Differential Equations and Dynamical Systems: Texts in A pplied Mathematics 7. ISBN-0387951164. Vol 7. Springer. New York. 2001

106. Phillips, Rob. Crystals, Defects and Microstructures: Modeling across scales. ISBN0521790050. Cambridge University Press. 2001

107. Psaras, Peter A and H Dale. Advancing Materials Research. ISBN-0309036976. Symposium in Washington DC 28-29 Oct 1985.

National Academy Press. Washington, DC. 1987

108. Rajchenbach, Jean. Stress Transmission Through a C ohesionless Material. http://www.ipme.ru/e-journals/MPM/no 1301/rajchenbach/rajchen.pdf Materials Physics and Mechanics. Vol 3.

Advanced Study Center Co. Ltd.. St.Petersburg, Russia. 2001

109. Rajeev, S G. Complex Analysis and Differential Equations: PH Y 401/ O PT 411. http://www.pas.rochester.edu/ rajeev/phy401/analysis.pdf

University of Rochester. Rochester, NY. December 12, 2001

110. Rechtorisz, Akos and Imre Bojtat, and Miklos Galos. Determination of Stress Intensity Factors on Rock Specimens.

http://www.vbt.bme.hu/phdsymp/2ndphd/proceedings/rechtorisz.pdf 
2nd International Ph.D. Symposium in Civil Engineering.

Technical University of Budapest. Budapest, Hungary. August 26-28, 1998

111. Rice, Matthew. A Symmetric Derivation of the Electromagnetic Energy-

Momentum Tensor. http://vortex.brynmawr.edu/vortex/eppaperrev4.pdf

Bryn Mawr College. Bryn Mawr, PA. April 2000

112. Roco, M C and S Williams, P Alivisatos. Vision for Nanotechnology Research and Development in the Next Decade: F undamental Sdentific Issues for N anotechnology.

http://www.wtec.org/loyola/nano/IWGN.Research.Directions http://www.wtec.org/loyola/nano/IWGN.Research.Directions/chapter02.pdf

Nanotechnology Research Directions: IWGN Workshop Report.

IWGN. Maryland. September 1999

113. Rothwell, Edward J. Lab \#3:: E lectrostatic Field C omputation.

http://www.egr.msu.edu/ rothwell/sim/306lab3.pdf

Michigan State University. East Lansing, MI. January 6, 2000

114. Sadoway, Donald R. The Imperfect Solid State: L ecture N otes $\mathrm{N} 06$.

http://web.mit.edu/3.091/www/archives/Notes 4.pdf

MIT. Massachusetts. September 1996

115. Sandwell, David T. Applications and Review of Fourier Transform/ Series.

http://topex.ucsd.edu/geodynamics/01 fourier.pdf

University of California San Diego. San Diego, CA. 2001

116. Saxe, John Godfrey. The Blind Men and the Elephant.

117. Schanz, Martin and Alexander H.D. Cheng. Compressional wav es in a one-

dimensional column. http://www.infam.tu-

braunschweig.de/ $\sim \mathrm{ms} /$ ictam poster.pdf

Technical University of Braunschweig. Braunschweig, Germany. 2001

118. Schiøtz, J and T. Vegge, F D Di Tolla, and K W Jacobsen. Atomic-scale Simulations of the Mechanical Deformation of Nanocrystalline Metals.

http://www.fysik.dtu.dk/ schiotz/papers/prb60 11971.pdf

Physical Review B. Vol 60, No 17.

The American Physical Society. Denmark. November 1, 1999 
119. Serway, Raymond A, Robert J Beichner, and John W Jewett, Jr. Physics for Scientists and Engineers. ISBN-0030226546. Saunders College Publishing. Orlando, FL. 2000

120. Smith, William F. Foundations of Materials Science and Engineering: Second E dition. Mc-Graw-Hill. New York, NY. 1993

121. Smith, Fred E. Software T ools for Dynamic C racking A nalysis.

Colorado State University. Fort Collins, CO. December 21, 2000

122. Sneiderman, Phil. Engineers C reate Superstrong and Ductile Copper: Extreme oold and high heat help optimize the metal's microstructure. http://www.jhu.edu/ gazette/2002/04nov02/04copper.html The Gazette Online. Vol 23, No 10. Johns Hopkins University. Baltimore, MD. November 2002

123. Sypeck, David J. Damage Evolution in Titanium Matrix Composites: Ph. D. D issertation. http://www.ipm.virginia.edu/research/Cons/thesis19.htm University of Virginia. VA. 1996

124. Székely, V and M. Rencz. Fast Field Solvers for Thermal and Electrostatic Analysis.

http://jamaica.ee.pitt.edu/Archives/ProceedingArchives/Date/Date98/papers/19 98/date98/pdffiles Budapest, Hungary.

125. Telling, R H and C J Pickard, M C Payne, and J E Field. Theoretical Strength and Cleavage of Diamond. Physical Review Letters. Vol 84, No 22. The American Physical Society. May 29, 2000

126. Thamm, Frigyes. The Role of Stress Trajectories as an Aid in the $\mathbf{C h o i c e}$ of the Suitable Shape: of L oad-bearing Structural E lements of E ngines and Structure. Mechanical Engineering. Vol. 44, No 1.

Periodica Polytechnica. Budapest, Hungary. June 30, 1999

127. Thompson, Jeffrey Y. Lecture: Bulk Properties. http://www.bme.unc.edu/ jyt/UUniversity of North Carolina. NC. 2001

128. Thompson, J M T and H B Stewart. Nonlinear Dynamics and Chaos: G eometrical Methods for E ngineers and Scientists. ISBN-0471909602.

John Wiley and Sons Ltd. New York. 1986, reprinted in 1989 
129. Thoroddsen, S T and Amy Q Shen. G ranular Jets.

http://www.tam.uiuc.edu/publications/tam reports/2000/941.pdf

Theoretical and Applied Mechanics (TAM) Reports. Urbana, Illinois. 2000

130. Timoshenko, Stephan P. History of Strength of Materials. ISBN-0486611876.

Dover Publications, Inc. New York. 1983

131. Tveito, Aslak and Ragnar Winther. Introduction to Partial Differential Equations: Texts in A pplied Mathematics 29. ISBN-0387983279. Vol 29. Springer. New York. 1998

132. Van, P and B Vasarhelyi. Second Law of Thermodynamics and the Failure of Rock Materials.

http://www.phy.bme.hu/ van/Publ/VanVas01p.pdf

Proceedings of the 38th U. S. Rock Mechanics Symposium. Rock mechanics in the national interest. Vol 1, 767-773.

Swets \& Zeitlinger Publishers. Budapest, Hungary. 2001

133. Veprek, Stan. The Search for Novel, Superhard Materials.

Journal of Vacuum Science \& Technology A. Vol 17 (5), 2401-2420.

American Vacuum Society. Munich, Germany. 1999

134. Wang, B T. Prediction of Impact and Harmonic Forces Acting on A rbitrary Structures: Theoretical Formulation.

http://www.sciencedirect.com

Mechanical Systems and Signal Processing. Vol. 16, No 6.

Elsevier Ltd. November 2002

135. Wangsness, Ronald. Electromagnetic Fields. ISBN-0471811566.

John Wiley \& Sons. New York. 1970, 1986

136. Weller, $\mathrm{R}$ and J K Bussey. Photoelastic Analysis of Three-Dimensional Stress using Scattered Light. http://naca.larc.nasa.gov/reports/1939/naca-tn-737/ Technical Notes. No. 737.

National Advisory Council for Aeronautics. Washington, D.C.. November 1939

137. Williams, CDH. A nalytic Solutions to Laplace's Equation. http://newton.ex.ac.uk/teaching/CDHW/EM/CW970317-2.pdf 1997

138. Wolfram, Stephen. A New Kind of Science. ISBN-1579550088.

Wolfram Media, Inc.. Champaign, IL. 2002 
139. Woolard, Deonna. O bstacles Encountered in C alculating the Stress Tensor:

U sing Thermoelasticity and Photoelastiaty.

http://www.udel.edu/physics/csaapt/Fall2000/powerpoint/deonna.ppt

Randolf-Macon College. Ashland, Virginia. Fall 2000

140. Wooster, W A. Tensor and Group Theory for the Physical Properties of Crystals. ISBN-019851137X. Oxford University Press. Oxford. 1973

141. Yosibash, Zohar. Finite Element Stress Extraction by the Complementary Energy Principle. International Journal for Numerical Methods in Engineering. Vol 40, 1335-1354. John Wiley \&Sons, Ltd. Israel. 1997

142. Zaslavsky, George M. From Hamiltonian C haos to Maxw ell's Demon.

Chaos. Vol. 5, No.4. American Institute of Physics. July 17, 1995

143. Zill, Dennis G. Calculus with A nalytic Geometry. ISBN-0534916201. PWSKent. Boston, MA. 2nd Ed 1988 